\title{
The luminous and dark matter content of disk galaxies ${ }^{\star}$
}

\author{
J. Zavala ${ }^{1}$, V. Avila-Reese ${ }^{1}$, H. Hernández-Toledo ${ }^{1}$, and C. Firmani ${ }^{2}$ \\ ${ }^{1}$ Instituto de Astronomía-UNAM, A.P. 70-264, 04510 México, D. F., México \\ e-mail: jzavala, avila,hector@astroscu.unam.mx \\ 2 Osservatorio Astronomico di Brera, via E. Bianchi 46, 23807 Merate, Italy \\ e-mail: firmani@merate.mi.astro.it
}

Received 18 March 2003 / Accepted 26 May 2003

\begin{abstract}
We have compiled a sample of disk galaxies with available photometry in the $B$ and $K$ bands, velocity line-widths and $\mathrm{HI}$ integral fluxes. Several parameters that trace the luminous, baryonic and dark matter contents were inferred. We investigated how these parameters vary with different galaxy properties, and compared the results with predictions of galaxy evolutionary models in the context of the $\Lambda$ Cold Dark Matter ( $\Lambda$ CDM) cosmogony. The ratio of disk-to-total maximum circular velocity, $\left(V_{\mathrm{d}, \mathrm{m}} / V_{\mathrm{t}, \mathrm{m}}\right)$, depends mainly on the central disk surface density $\Sigma_{\mathrm{d}, 0}$ (or surface brightness, $\mathrm{SB}$ ), increasing roughly as $\Sigma_{\mathrm{d}, 0} 0.15$. While a fraction of high SB galaxies have a $\left(V_{\mathrm{d}, \mathrm{m}} / V_{\mathrm{t}, \mathrm{m}}\right)$ ratio corresponding to the maximum disk solution, the low $\mathrm{SB}$ are completely dark matter dominated. The trend is similar for the models, although they have slightly smaller $\left(V_{\mathrm{d}, \mathrm{m}} / V_{\mathrm{t}, \mathrm{m}}\right)$ ratios than observations, in particular at the highest SBs and when small baryon fractions are used. The scatter in the $\left(V_{\mathrm{d}, \mathrm{m}} / V_{\mathrm{t}, \mathrm{m}}\right)-\Sigma_{\mathrm{d}, 0}$ plot is large. An analysis of residuals shows that $\left(V_{\mathrm{d}, \mathrm{m}} / V_{\mathrm{t}, \mathrm{m}}\right)$ tends to decrease as the galaxy is redder, more luminous (massive), and of earlier type. The models allow us to explain the physics of these results, which imply a connexion between halo structure and luminous properties. The dynamical-to-baryon mass and dynamical mass-to-light ( $B$ and $K$ ) ratios at a given radius were also estimated. All these ratios, for observations and models, decrease with $\Sigma_{\mathrm{d}, 0}$ (or $\mathrm{SB}$ ) and do not correlate significantly with the galaxy scale, contrary to what has been reported in previous works, based on the analysis of rotation curve shapes. We discuss this difference and state the importance of solving the controversy of whether the dark and luminous contents in disk galaxies depend on SB or luminosity. The broad agreement between the models and observations presented here regarding the trends of the dynamical-to-baryon matter and mass-to-light ratios with several galaxy properties favors the $\Lambda$ CDM scenario. However, the excess of dark matter inside the optical region of disk galaxies remains the main difficulty.
\end{abstract}

Key words. cosmology: dark matter - galaxies: evolution - galaxies: fundamental parameters - galaxies: halos

\section{Introduction}

The information about the distribution of luminous and dark matter in disk galaxies, as well as on the correlations among the main parameters that characterize both components, offers a fundamental clue to understand how galaxies form and evolve and what role dark matter plays in these processes. Current models of disk galaxy formation and evolution, based on the hierarchical Cold Dark Matter (CDM) scenario, make certain predictions about these distributions and correlations (e.g., Firmani et al. 1997; Dalcanton et al. 1997; Mao \& White 1998; Avila-Reese et al. 1998; van den Bosch 1998, 2000; Firmani \& Avila-Reese 2000; Avila-Reese \& Firmani 2000). These predictions can be used to confront and interpret the available observations. This is the aim of the present paper, where we will compare some global properties and correlations of observed galaxies with the model ones. From the point of view of the

\footnotetext{
Send offprint requests to: V. Avila-Reese, e-mail: avila@astroscu.unam.mx

* Appendices A and B are only available in electronic form at http://www.edpsciences.org. Table 1 is only available at the CDS via anonymous ftp to cdsarc.u-strasbg. fr (130.79.128.5) or via
}

http://cdsweb.u-strasbg.fr/cgi-bin/qcat?J/A+A/412/633 models, the galaxy properties are the result of the combination of some fundamental (cosmological) parameters, which follow continuous statistical distributions. Therefore, an observational sample as complete as possible in luminosities, surface brightnesses, morphological types and integral colors is crucial.

More than 25 years ago, the interpretation of the observations under the assumption of Newtonian dynamics suggested the presence of dark matter in and around disk galaxies (Bosma 1978, 1981a, 1981b; Rubin et al. 1980, 1982, 1985; see also Rogstad \& Shostak 1972). Two relevant (related) questions are whether the disk dominates the mass within some parts of the galaxies or not, and whether the dark halo is shallow or cuspy in the center (for recent reviews see e.g., Bosma 2002 and Salucci $\&$ Borriello 2001). The latter issue turned out to be crucial for testing the predictions of CDM models, and it is investigated currently by studying the inner rotation curves of observed dwarf and low surface brightness (LSB) galaxies, which are dark matter dominated systems.

The rotation curve decomposition of individual galaxies was commonly used to explore the fraction of dark matter within them (e.g., Carignan \& Freeman 1985; van Albada \& Sancisi 1986; Kent 1986). Besides postulating a stellar massto-light ratio at the observed band, $\gamma_{\lambda}$, this method requires one to assume a dark halo mass distribution for the given galaxy. 
A popular technique is to fix $\gamma_{\lambda}$ (assumed constant with radius) at the highest possible value such that the "luminous" rotation curve component does not generate a higher rotation velocity than actually observed in the inner part, the so called "maximum disk" hypothesis; the complement to the observed rotation curve is fitted to the assumed non-hollow dark halo model (typically a pseudo-isothermal sphere). For a maximum disk model, the disk velocity component at its maximum dominates over the halo. Luminous and high surface brightness (HSB) galaxies are typically well described by the maximum disk model, while this model for low luminosity and LSB galaxies implies unrealistically high stellar mass-to-light ratios (see references in Sect. 3.4). The fractions of luminous and dark matter inferred with the individual velocity decomposition methods are model-dependent and in certain circumstances the model may converge to an erroneous solution or to a non-reasonable values for $\gamma_{\lambda}$ (Persic \& Salucci 1991). Besides, for these methods detailed photometric and kinematic data are required.

The fractional amount of dark matter in disk galaxies have been also inferred by studying the kinematical properties of a set of rotation curves (Persic \& Salucci 1988, 1990a; Salucci \& Persic 1999). The virtue of this method is that no assumption about a particular dark matter distribution needs to be done. Solely from the shape of the rotation curves and the definition of some radial scale connected to the luminous distribution (assumed exponential), it is possible to infer on average the fraction of dark and luminous matter at this scale. Persic \& Salucci have found a dependence of the rotation curve slope measured at $3.2 h_{\mathrm{d}}\left(h_{\mathrm{d}}\right.$ is the total disk scale radius assumed equal to the optical scale radius) with the optical luminosity of the galaxy, which translates into a dependence of the disk-to-total mass ratio at $3.2 h_{\mathrm{d}}$ on luminosity: $M_{\text {disk }} / M_{\text {tot }} \propto L_{\mathrm{B}}^{0.4}$. The samples used by these authors included only HSB highly-inclined late-type spirals with available $\mathrm{H} \alpha$ rotation curves and optical photometry.

A major goal of the mass modeling method based on the shape of rotation curves was the inference of the so-called "universal rotation curve" (Persic et al. 1996). From about one thousand observed $\mathrm{H} \alpha$ rotation curves of late-type HSB spirals, synthetic rotation curves binned by luminosity intervals were generated. Assuming an exponential disk and a halo with a velocity profile proportional to $r /\left(r^{2}+a^{2}\right)^{1 / 2}$, where $a$ is a core radius, the synthetic rotation curves could be well described by an universal profile, resulting from the quadratic sum of the disk and halo velocity components. The two parameters of this profile are the disk-to-total velocity ratio at $3.2 h_{\mathrm{d}}$ and the core radius. Persic et al. found that, in order that the synthetic rotation curves could be fitted with the universal profile, these parameters, in particular the former, should be a function of luminosity. Therefore, the universal rotation curve is a function of luminosity in the sense that as the galaxy is less luminous, the larger the slope of the rotation curve at $3.2 h_{\mathrm{d}}$ becomes. A direct implication of this result (which was in fact the inspiration for it) is that the dark-to-luminous mass ratio (at $3.2 h_{\mathrm{d}}$ ) scales inversely with luminosity.

In spite of the virtues of the rotation curve shape method to infer dark and luminous mass fractions, it is not free of uncertain assumptions (to be discussed in Sect. 4.2), which could bias the results. Moreover, other workers have shown several examples of rotation curves that deviate from the universal curve (Verheijen 1997; Bosma 1998), suggesting that besides the luminosity, other observational parameters, for example the surface brightness (SB), could be important, and therefore, the disk-to-total mass ratio could also depend on them. Thus, it is important to explore alternative methods based on more complete samples, in the sense of SBs, luminosities, and morphological types, to infer the dark and luminous fractions in disk galaxies.

Some of these alternative methods are based on observed global galaxy parameters and relationships among them rather than in the local kinematics (e.g., Salucci et al. 1991). In the present paper, we follow this kind of methods, inferring from the observations (i) global parameters related to the amounts of luminous and dark matter in disk galaxies, and (ii) exploring how these parameters vary with galaxy properties. Part of the analysis presented here is closely related to the fundamental plane of disk galaxies. However, a direct study of the fundamental plane will be presented elsewhere. The results obtained will be compared with predictions of galaxy evolution models in the hierarchical clustering scenario, in order to test whether disks formed inside Cold Dark Matter (CDM) halos are realistic or not.

A prediction of this scenario is that the luminous-to-dark mass ratio within the optical parts of the disks depends mainly on the disk surface density and on the global disk(baryon)-tohalo mass fraction, $f_{\mathrm{d}}{ }^{1}$ (Firmani \& Avila-Reese 2000; see also Dalcanton et al. 1997; Mo et al. 1998). A dependence on the mass (or luminosity) is not expected, unless astrophysical process, like feedback, introduce a strong dependence of $f_{\mathrm{d}}$ on the mass. Recent calculations by van den Bosch (2002) show that the feedback reduces $f_{\mathrm{d}}$ in low massive galaxies but there is not a significant trend with the mass. In fact, in massive galaxies not all the baryon fraction ends in the disk due to a long gas cooling time in these systems, so that both low mass and massive systems on average incorporate $0.4-0.6$ of the halo baryon fraction to the disk (van den Bosch 2002), without any significant dependence on mass (i.e., $f_{\mathrm{d}}$ is not expected to depend on mass).

In spite of the great observational effort done in the last decades, there are actually few sufficiently homogeneous observational works reporting both photometric (optical and nearinfrared) and kinematic information, including HI detection, for disk galaxies. In this paper, we present an extensive compilation from the literature for HSB and LSB disk galaxies with photometric parameters in both the $B$ and $K$ bands, rotation curves or velocity line-widths and $\mathrm{HI}$ integral fluxes (see also Graham 2002). After applying a uniforming procedure, the compiled data is used to estimate several stellar and baryonic parameters of the disks. The most reliable data for this are in the near-infrared bands $(H$ or $K)$. The sample and the results in this and in a forthcoming paper can be used as a local reference

1 We define $f_{\mathrm{d}} \equiv M_{\mathrm{bar}} / M_{\mathrm{v}}$, where $M_{\mathrm{bar}}$ is the total baryonic galaxy mass, referred some times also as the disk mass, $M_{\mathrm{d}}$, and $M_{\mathrm{v}}$ is the virial (total) halo mass. 
for studies of the luminous and dynamical properties of disks in other environments and at higher redshifts.

In Sect. 2, we present the compiled sample and describe the procedures to make the data uniform and to estimate stellar and baryonic parameters from the observations. Section 3 is devoted to the study of the ratio of disk-to-total maximum velocity at their corresponding maxima, $\left(V_{\mathrm{d}, \mathrm{m}} / V_{\mathrm{t}, \mathrm{m}}\right)$. This quantity is not defined at a given radius and gives an approximate estimate of the dark and luminous fractions. For the kind of kinematical data we use here (equivalent line-width instead of detailed rotation curve $),\left(V_{\mathrm{d}, \mathrm{m}} / V_{\mathrm{t}, \mathrm{m}}\right)$ is the most direct quantity to compare with models, without introducing extra assumptions. In Sect. 3.1 simple composite halo/disk models predictions are presented, while in Sect. 3.2 the description and results for semi-numerical galaxy evolution models in the $\Lambda \mathrm{CDM}$ scenario are given. In Sects. 3.3 and 3.4, the $\left(V_{\mathrm{d}, \mathrm{m}} / V_{\mathrm{t}, \mathrm{m}}\right)$ ratio of observed and model galaxies and the dependence of this ratio on several galaxy parameters are presented and compared. In the light of the results obtained, the issue of maximum or sub-maximum disk is discussed. In Sect. 4, the dynamical-tobaryonic mass ratio (here instead of "total" we use the term "dynamical"), $M_{\mathrm{dyn}} / M_{\mathrm{bar}}$, and the mass-to-light ratios (both measured at some inner radius) for models and observations, as well as their dependences on several galaxy parameters are presented. For the observational data, some assumptions should be made in order to estimate these ratios; we explore how much these assumptions could affect our conclusions. In Sect. 4.2, we compare our results with those in previous works and discuss the differences. Finally, a summarizing discussion is given in Sect. 5.

\section{The sample}

In order to trace the baryonic and dark matter content of disk galaxies it is important to explore as wide range of galaxy types, magnitudes, sizes, and surface brightnesses as possible. Furthermore, high-quality surface photometry in the optical and near-IR pass-band as well as information about the rotation curve or at least the $\mathrm{HI}$ or $\mathrm{H} \alpha$ line-width and the total HI gas flux are needed. After an extensive search of the literature, we surprisingly have found only a few (sufficiently homogeneous) observational sources to compile a sample of disk galaxies with the required data (see also Graham 2002). From these sources, three main sub-samples of HSB and LSB galaxies were collected:

(1) de Jong sub-sample (de Jong \& van der Kruit 1994; de Jong 1996a) reports bidimensional $B, V, R, I$ and $K$-band photometry for 86 undisturbed field spirals selected from the Uppsala General catalogue (UGC). Only galaxies classified equal or later than S1 or SB1 and with an inclination limit $\leq 51^{\circ}$ were included. This is a diameter-limited sample (UGC is expected to be complete up to 2 arcmin red diameters) that can be transformed into a volume-limited sample.

(2) Verheijen (1997) sub-sample (see also Verheijen \& Sancisi 2001) is a selection of galaxies from the Ursa Major Cluster. This is one of the least massive and most spiralrich nearby clusters. The sample is complete for galaxies later than Sab to a limiting apparent Zwicky magnitude $m z w=$ 15.2, although it includes galaxies fainter than that cutt-off.
It contains $B, V, R, J, K^{\prime}$-band photometry and kinematic (HI observations) for HSB and LSB disk galaxies. The kinematic data were obtained by the authors only for galaxies (52) with inclinations greater than $45^{\circ}$ and include synthetic rotation curves as well as equivalent line-widths. Notice that although it is suspected that cluster environment affect the properties of the constituent galaxies, in the case of the Ursa Major, the cluster may be so young that its members are more representative of a field population.

(3) Bell et al. (2000) sub-sample, obtained partially by themselves and compiled partially from the literature, mainly from de Blok et al. (1995) and de Blok et al. (1996), with the condition to have blue central surface brightness $\mu_{B, o} \geq 22.5 \mathrm{mag} \mathrm{arcsec}^{-2}$ and diameters at the $25 \mathrm{mag} \mathrm{arcsec}^{-2}$ isophote larger than $16^{\prime \prime}$. Their sample consist of 26 LSB galaxies with available bidimensional $B$ - and $K$-band photometry and an inclination limit $\leq 61^{\circ}$. The sample is by no means complete but it is designed instead to span as wide a range as possible of observed LSB galaxy parameters. Notice that although LSB galaxies follow the spatial distribution of HSB galaxies, they tend to be more isolated from their nearest neighbours than HSB galaxies (cf. Bothun et al. 1993).

The Milky Way and Andromeda galaxies were also included so that a direct comparison of their properties w.r.t. the galaxies in our sample could be inferred.

The galaxies from the Verheijen sub-sample and some LSB galaxies from the Bell et al. sub-sample have measured synthetic HI rotation curves. Thus, their maximum total velocity rotation, $V_{\mathrm{t}, \mathrm{m}}$, is known. For the rest of the galaxies in our collected sample, only equivalent velocity line-widths are reported. In order to make the data as uniform as possible, we used the velocity line-width $W_{20}$ (defined at the 20\% level) for all the galaxies in the sample. Only a few (LSB) galaxies in the sample lack a $W_{20}$ measurement but have measured rotation curves; in these cases we have used the $V_{\mathrm{t}, \mathrm{m}}$ inferred directly from the rotation curve. If properly corrected, $W_{20}$ is $\approx 2 V_{\mathrm{t}, \mathrm{m}}$ as Verheijen (1997, p. 197) have shown (see also Verheijen \& Sancisi 2001). Thus, the dynamical information available for our sample will be $V_{\mathrm{t}, \mathrm{m}}$. The radius where $V_{\mathrm{t}}$ peaks, symbolized here as $r_{\text {peak }}$, is not obviously related to the typical radius of the disk but it can be estimated as a multiple of $h_{\mathrm{d}}$ (see Sect. 3). For the Verheijen sub-sample, we adopt his $W_{20}$ values, while for the de Jong sub-sample and most of the LSB galaxies from the Bell et al. sub-sample, $W_{20}$ was extracted from the Lyon Extragalactic Database (LEDA) database ${ }^{2}$. The integral fluxes in the 21-cm line (used to calculate the neutral gas masses in galaxies) were taken from Verheijen \& Sancisi (2001), LEDA, and de Blok et al. (1996).

A condition imposed on our sample is that galaxies should be in a restricted range of inclinations $\left(35^{\circ} \leq i \leq 80^{\circ}\right)$. Otherwise either the surface brightness (hereafter SB) profiles or the rotation velocities are less reliable. The inclinations were calculated from the photometric minor-to-major semiaxis ratios (typically in a near-infrared band) reported in the source papers and assuming a fixed thickness parameter $q=$ 0.2. We exclude from the sample galaxies with clear signs of

\footnotetext{
${ }^{2}$ http://leda.univ-lyon $1 . f r$
} 
interaction (mainly from the Verjeihen sub-sample) and with rotation curves that are still increasing at the last measured outer point. Only some LSB galaxies showed this feature. Fortunately, there are published synthetic rotation curves for most of the LSB galaxies in our sample. The final sample consists of 78 galaxies: 42 out of 86,29 out of 52 , and 5 out of 23 from the de Jong, Verheijen and Bell et al. sub-samples, respectively, plus the MW and Andromeda galaxies. The Bell et al. sample contains 26 LSB galaxies, but three of them were taken from the de Jong sample.

The local distances to the galaxies were calculated using the kinematic distance modulus given in LEDA. The value of the Hubble constant used in this database is the same we assume here, $H_{0}=70 \mathrm{~km} \mathrm{~s}^{-1} \mathrm{Mpc}^{-1}$. Since the LSB galaxies from the Bell et al. sub-sample are not included in LEDA, their distances were taken directly from Bell et al. (2000) correcting them for the $H_{0}$ value used here. From the given distances, the corresponding redshifts were obtained. Using these redshifts, we then calculated the luminous distances as: $D_{\mathrm{L}}=\frac{c z}{H_{0}}\left[1+\frac{1}{2}\left(1-q_{0}\right) z\right]$, where $q_{0}$ is the deceleration parameter and $\mathrm{c}$ is the speed of light. For the cosmology used in this paper $\left(\Omega_{m}=0.3, \Omega_{\Lambda}=0.7\right), q_{0}=-0.55$. The above equation is a good approximation for small redshifts.

Merits and limitations of the sample. The sample is not complete in any sense, but it is representative of the basic parameters required: morphological type, luminosity, SB and color. In Fig. 1, the number distribution of morphological types, corrected $B$-band magnitudes and SBs, and corrected $(B-K)$ colors are presented (for the corrections, see Sect. 2.1). The number distribution of types is similar to that in other samples of nearby galaxies (cf., Hernandez-Toledo et al. 1999; Jansen et al. 2000), with a high peak at $T=4,5$. The distribution of absolute magnitudes indicates that the sample is representative of the local galaxy luminosity function in the bright part $\left(M_{\mathrm{abs}} \lesssim\right.$ -19.5 ) but lacks enough representation for fainter luminosities. In fact, the faint part of the luminosity function consists mostly of dwarf galaxies. The physics of this group of galaxies is different to that of normal disk galaxies. The aim of the present paper is to investigate only the latter ones. The SB distribution presents a bimodality (Tully \& Verheijen 1997). The sample is not complete at very LSBs. This is consistent with the lack of faint galaxies in the magnitude number distribution: very LSB galaxies are typically dwarf galaxies.

Since we adopt the raw disk central SB and scale radius $\left(\mu_{\lambda, 0}\right.$ and $\left.h_{\lambda}\right)$ directly from the above source samples, it is important to check that the the various methods used to infer these parameters do not introduce systematic differences in our compiled sample data. For the de Jong sample, a two-dimensional exponential bulge-bar-disc decomposition was used (de Jong 1996). Bell et al. (2000) used a onedimensional bulge-disc decomposition (with either an exponential or an $r^{1 / 4}$ law bulge). Verheijen used a "marking the disc" fit, where the contribution of the bulge to the disc parameters is minimized by visually assessing where the contribution from the bulge component is negligible. De Jong (1996a) showed that the one- and two-dimensional methods give comparable results to typically better than $20 \%$ in terms of surface
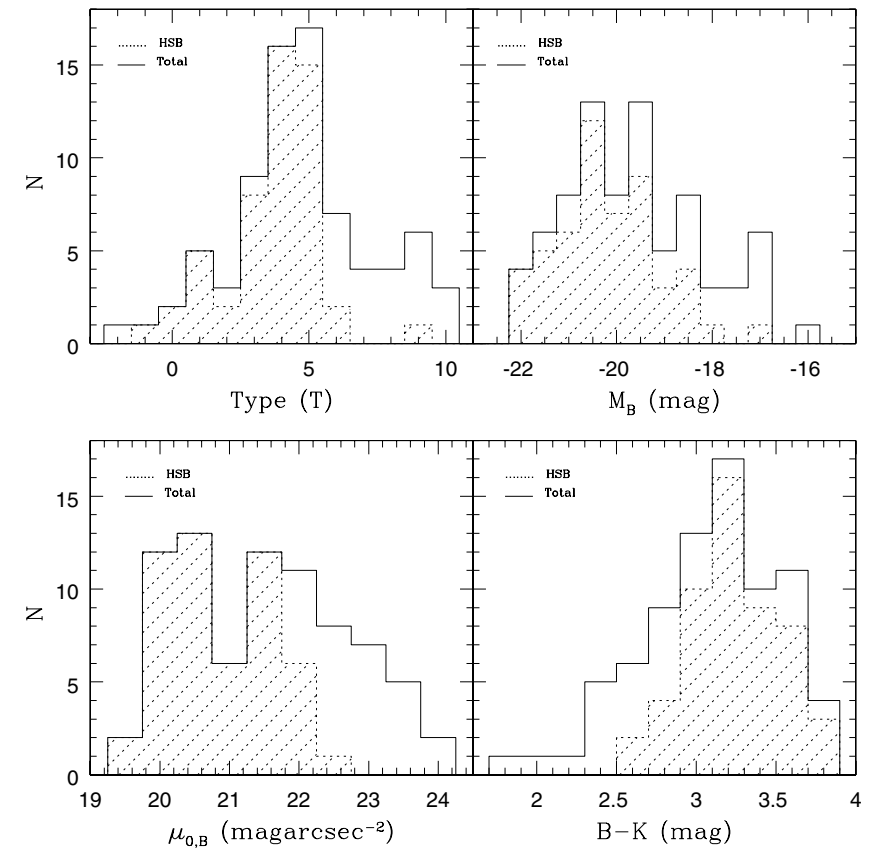

Fig. 1. Number distributions of morphological types, $B$-band corrected absolute magnitudes and $\mathrm{SBs}$, and corrected $(B-K)$ colors for the recompiled sample of 78 normal HSB and LSB disk galaxies. The contribution of the HSB galaxies is shaded.

brightness (SB hereafter) and 10\% in terms of scalelengths, with little significant bias.

After a comparison of the de Jong and Verheijen samples in the $L_{\lambda}-h_{\lambda}$ diagram for the $B$ and $K$ bands, we found no systematic differences. Notice that the selection criteria applied in de Jong (1996a) implies that small LSB galaxies are excluded. This selection effect could introduce a systematic difference to the Verjeihen galaxies. However, only a few galaxies from the latter author fall below the selection limit of de Jong in the $\mu_{K, 0^{-}}$ $h_{K}$ diagram $\left(H_{0}=70 \mathrm{~km} \mathrm{~s}^{-1} \mathrm{Mpc}^{-1}\right.$; this value is used throughout the paper). We also checked that the scale radii reported by these authors do not correlate with inclination (e.g., Giovanelli et al. 1984). When the bulge is not taken into account in the fitting, such a correlation could appear, but that is not the case for the Verheijen data.

In conclusion, we do not find significant systematic differences in $\mu_{\lambda, 0}$ and $h_{\lambda}$ among the galaxy sub-samples due to the different profile fitting procedures used. This is consistent with the de Jong (1996a) conclusion mentioned above. In fact, the dominant source of error in the determination of the galaxy photometric parameters is due to the uncertainty in the sky level (Bell et al. 2000; de Jong 1996a).

\subsection{Data corrections}

The total magnitudes were corrected for Galactic extinction (Schlegel et al. 1998), $K$-correction (Poggianti 1997), and internal extinction. Since the fraction of dust seems to be larger for bigger galaxies according to empirical (e.g., Wang \& Heckman 1996; Tully et al. 1998) and theoretical (e.g., Shustov et al. 1997) arguments, we used the empirical velocity(luminosity-) dependent extinction coefficients determined by Tully et al. (1998) for a large sample of galaxies (HSB and 
LSB) in clusters. The $K^{\prime}$-band data from the Verheijen subsample were transformed into $K$-band adopting $K^{\prime}-K=$ $0.19(H-K)$ (Wainscoat \& Cowie 1992) and the average $(H-K)$ color of each morphological type given in de Jong (1996c).

The SBs were corrected for Galactic extinction, $K$-correction, cosmological SB dimming, and inclination (geometrical and extinction effects). For the latter correction, we followed Verheijen (1997), considering LSB galaxies as optically thin in all bands. The HSB galaxies were also considered as optically thin in the $K$-band. We have defined LSB galaxies as those whose disk central SB in the $K$-band after SB correction is larger than $18.5 \mathrm{mag} / \mathrm{arcsec}^{2}$ (Verheijen 1997).

The $21 \mathrm{~cm}$ line-widths at the $20 \%$ level, $W_{20}$, for the Verheijen susbsample were taken directly from Verheijen \& Sancisi (2001). Galaxies with $W_{20}$ taken from LEDA, were de-corrected (for instrumental corrections) to get the raw data (Paturel et al. 1997) and then, corrected again for broadening due to turbulent motions and for inclination, this time following Verheijen \& Sancisi (2001).

The final sample data are presented in Table 1 available at the CDS. The name and morphological type are given in Col. 1 . The luminous distance and the galaxy inclination are presented in Col. 2. Raw apparent magnitudes and corrected luminosities in the $B$ and $K$ bands appear in Cols. 3 and 4, respectively. The solar magnitudes used were $M_{B_{\odot}}=5.48$ and $M_{K_{\odot}}=3.41$. The raw and corrected central SBs in the $B-$ and $K$-bands appear in Cols. 5 and 6 , while Col. 8 shows the corresponding disk scale radii (in kpc). In Col. 7, the corrected integral and central $(B-K)$ colors are given. Finally, Col. 9 gives the corrected line-widths at the $20 \%$ level, $W_{20}$, and the HI mass, $M_{\mathrm{HI}}\left(M_{\odot}\right)=$ $2.36 \times 10^{5} D_{\mathrm{L}}^{2}(\mathrm{Mpc}) \int S_{v}\left(\mathrm{Jy} \mathrm{km} \mathrm{s}^{-1}\right) \mathrm{d} v$.

\subsection{Composite quantities}

The stellar mass and disk central surface density, $M_{\mathrm{s}}$ and $\Sigma_{\mathrm{s}, 0}$, are derived from the $K$-band luminosity (which includes the bulge) and central SB, respectively. It seems that, even in the $K$ band, the stellar mass-to-light ratio, $\gamma_{K}$, is not constant. The application of population synthesis techniques to simplified galaxy evolution models for HSB galaxies show that $\gamma_{K}$ depends mainly on the integral color (Tinsley 1981; Bruzual 1983; Bell \& de Jong 2001). From Table 1 (model "formation epoch with bursts (vi)") in Bell \& de Jong (2001), we infer

$\log \gamma_{K}=-0.91+0.21(B-K)$.

For low SBs this relation breaks down. The various rotation curve decomposition models applied by Verheijen (1997) to his sample of LSB galaxies, actually shows that $\gamma_{K}$ increases toward bluer galaxies. A linear eye fit to the $\gamma_{K}-(B-K)$ relation of his LSB galaxies, assuming a Hernquist halo model and using his constrained decomposition method, gives

$$
\begin{array}{cc}
\gamma_{K}=2.75-0.75\left(B-K^{\prime}\right), & \left(B-K^{\prime}\right) \lesssim 3 \\
\gamma_{K}=0.5, & \left(B-K^{\prime}\right)>3 .
\end{array}
$$

This result is similar to that inferred from other decomposition methods and for the pseudo-isothermal halo used by
Verheijen (1997; see Fig. 10 in Chap. 6). The scale radius of the stellar disk, $h_{\mathrm{s}}$, is assumed here to be equal to the scale radius in the band $K, h_{K}$. Most of the stars in the disk are traced in this band.

The disk gas mass, $M_{\mathrm{g}}$, is estimated as

$$
M_{\mathrm{g}}=1.4 M_{\mathrm{HI}}\left[1+\frac{M_{\mathrm{H}_{2}}}{M_{\mathrm{HI}}}\right]
$$

where the factor 1.4 takes into account helium, and $M_{\mathrm{H}_{2}}$ is the molecular hydrogen mass. The $M_{\mathrm{H}_{2}} / M_{\mathrm{HI}}$ ratio has been found to depend on the morphological type $T$ (Young \& Knesek 1989). From the latter paper, McGaugh \& de Blok (1997) estimated that $M_{\mathrm{H}_{2}} / M_{\mathrm{HI}}=3.7-0.8 T+0.043 T^{2}$. For $T<2$ this empirical fitting formula overestimates strongly the gas mass in galaxies, thus we assume $M_{\mathrm{H}_{2}}=0$.

The estimation of the gas and baryonic galaxy parameters is important to understand several structural and dynamical aspects of galaxies and to compare with the theoretical predictions. The galaxy baryonic mass is $M_{\mathrm{bar}}=M_{\mathrm{s}}+M_{\mathrm{g}}$, and the gas mass fraction is defined as $f_{\mathrm{g}}=M_{\mathrm{g}} / M_{\mathrm{bar}}$. Unfortunately, we do not have information about the gas surface density profiles $\left(\Sigma_{\mathrm{g}, 0}\right.$ and $\left.h_{\mathrm{g}}\right)$ for all galaxies in our sample. We need them in order to calculate the baryonic disk central surface densities and scale radii. The observed density profiles of neutral gas in disk galaxies are diverse and there seems to exist a characteristic profile for each morphological type (Broeils \& van Woerden 1994). The HI distribution in the center of Sb-Scd galaxies is typically flat or even has a hollow, but the $\mathrm{H}_{2}$ distribution for these galaxies rapidly increases to the center compensating the HI deficiency. For early-type galaxies, the HI profile is roughly exponential and $\mathrm{H}_{2}$ is deficient. In a very rough approximation, the total gas distribution $\left(\mathrm{HI}+\mathrm{H}_{2}\right)$ in disk galaxies can be described by an exponential profile with scale radii 2.0-3.0 times larger than the stellar scale radii in the near-infrared bands (e.g., Olivier et al. 1991; Marsh \& Helou 1995). We will assume that the total gas surface density follows an exponential distribution with an scale radius 3 times $h_{K}$. Thus, $\Sigma_{\mathrm{g}, 0}=M_{\mathrm{g}} / 2 \pi\left(3 h_{K}\right)^{2}$. The baryonic disk central surface density is then calculated as $\Sigma_{\mathrm{d}, 0}=\Sigma_{\mathrm{s}, 0}+\Sigma_{\mathrm{g}, 0}$. The corresponding baryonic disk scale radius will be $\left.h_{\mathrm{d}}=h_{K}\left[\left(\Sigma_{\mathrm{s}, 0}+9 \Sigma_{\mathrm{g}, 0}\right) / \Sigma_{\mathrm{d}, 0}\right)\right]^{0.5}$. In fact, our main results in Sect. 3 will not be affected by including the gas disk parameters, but some influence is expected. This is why we tried to use the information we have at least about the global gas content, by introducing an "effective" gas scale radius and surface density.

In Table 2 we present the composite quantities described above for the sample galaxies in Table 1. Columns 2 and 3 contain the disk stellar mass and central surface density $\left(M_{\mathrm{S}}\right.$ and $\left.\Sigma_{\mathrm{s}, 0}\right)$. Columns 4 and 5 the total disk gas mass and mass gas fraction $\left(M_{\mathrm{g}}\right.$ and $\left.f_{\mathrm{g}}\right)$. Finally, Cols. 6, 7 and 8 list disk baryonic mass, central surface density and scale radius $\left(M_{\mathrm{bar}}, \Sigma_{\mathrm{d}, 0}\right.$, and $h_{\mathrm{d}}$, respectively).

\section{Disk-to-total maximum velocity ratio}

We are interested in exploring the mass amounts of luminous and dark matter in disk galaxies and how these amounts do depend on different galaxy properties. Our goal is to compare 
Table 2. Inferred stellar, gaseous, and baryonic parameters.

\begin{tabular}{|c|c|c|c|c|c|c|c|c|c|c|c|}
\hline Name & $\operatorname{Lg} M_{\mathrm{s}}{ }^{a}$ & $\operatorname{Lg} \Sigma_{\mathrm{s}, 0}{ }^{b}$ & $\operatorname{Lg} M_{\mathrm{g}}{ }^{c}$ & $\operatorname{Lg} \Sigma_{q}^{d}$ & $f_{g}^{e}$ & $\operatorname{Lg} M_{\mathrm{bar}}{ }^{f}$ & $\operatorname{Lg} \Sigma_{\mathrm{d}, 0}{ }^{g}$ & $h_{\mathrm{d}}{ }^{h}$ & $\left(V_{\mathrm{d}, \mathrm{m}} / V_{\mathrm{t}, \mathrm{m}}\right)^{i}$ & $M_{\mathrm{dyn}} / M_{\mathrm{bar}^{j}}{ }^{j}$ & $M_{\mathrm{dyn}} / L_{B}{ }^{k}$ \\
\hline UGC 89 & 11.33 & 2.63 & 10.11 & 0.84 & 0.06 & 11.35 & 2.64 & 6.06 & 0.64 & 2.08 & 4.79 \\
\hline UGC 242 & 10.67 & 2.74 & 10.06 & 1.22 & 0.20 & 10.77 & 2.75 & 3.87 & 0.74 & 3.21 & 6.94 \\
\hline UGC 334 & 9.99 & 1.49 & 9.94 & 0.63 & 0.47 & 10.26 & 1.54 & 8.46 & 0.59 & 4.69 & 10.92 \\
\hline UGC 438 & 11.25 & 3.22 & 10.31 & 1.41 & 0.10 & 11.29 & 3.23 & 3.95 & 0.97 & 1.75 & 5.36 \\
\hline UGC 490 & 11.12 & 2.83 & 10.57 & 1.45 & 0.22 & 11.23 & 2.84 & 5.56 & 0.85 & 2.13 & 8.08 \\
\hline UGC 628 & 10.04 & 1.47 & 9.96 & 0.49 & 0.45 & 10.30 & 1.51 & 9.54 & 0.44 & 9.40 & 22.08 \\
\hline UGC 1305 & 10.99 & 2.73 & 10.10 & 0.94 & 0.11 & 11.04 & 2.74 & 5.34 & 0.68 & 3.73 & 13.98 \\
\hline UGC 1577 & 10.92 & 2.28 & 10.69 & 1.29 & 0.37 & 11.12 & 2.32 & 8.79 & 0.65 & 3.48 & 11.31 \\
\hline UGC 1719 & 11.37 & 2.58 & 10.71 & 1.12 & 0.18 & 11.46 & 2.59 & 9.38 & 0.57 & 4.55 & 14.50 \\
\hline UGC 2064 & 10.72 & 2.44 & 10.30 & 1.10 & 0.28 & 10.86 & 2.46 & 6.17 & 0.83 & 2.66 & 7.59 \\
\hline UGC 2081 & 9.78 & 1.87 & 9.64 & 0.81 & 0.42 & 10.01 & 1.90 & 4.43 & 0.56 & 5.87 & 13.14 \\
\hline UGC 4308 & 10.63 & 2.55 & 10.33 & 1.35 & 0.34 & 10.81 & 2.57 & 5.03 & 0.76 & 3.14 & 6.42 \\
\hline UGC 4368 & 10.56 & 2.51 & 10.35 & 1.38 & 0.38 & 10.77 & 2.54 & 5.03 & 0.73 & 3.43 & 8.02 \\
\hline UGC 4375 & 10.34 & 2.72 & 9.66 & 1.12 & 0.17 & 10.42 & 2.73 & 2.71 & 0.70 & 3.78 & 9.04 \\
\hline UGC 4422 & 11.16 & 2.25 & 10.51 & 0.92 & 0.18 & 11.25 & 2.27 & 9.65 & 0.45 & 5.82 & 16.67 \\
\hline UGC 5103 & 10.95 & 2.90 & 10.55 & 1.63 & 0.29 & 11.09 & 2.92 & 4.57 & 0.86 & 2.33 & 4.97 \\
\hline UGC 5303 & 10.32 & 2.36 & 9.78 & 0.86 & 0.22 & 10.43 & 2.38 & 4.26 & 0.62 & 5.03 & 9.67 \\
\hline UGC 5510 & 9.99 & 2.66 & 9.82 & 1.57 & 0.40 & 10.21 & 2.70 & 2.24 & 0.75 & 3.30 & 6.22 \\
\hline UGC 5554 & 10.12 & 2.88 & 8.83 & 0.72 & 0.05 & 10.14 & 2.88 & 1.56 & 0.63 & 4.17 & 10.48 \\
\hline UGC 5633 & 9.39 & 1.68 & 9.36 & 0.67 & 0.48 & 9.67 & 1.72 & 3.83 & 0.46 & 9.33 & 23.44 \\
\hline UGC 6028 & 9.41 & 2.49 & 9.71 & 1.91 & 0.67 & 9.89 & 2.59 & 1.73 & 0.72 & 3.56 & 7.63 \\
\hline UGC 6453 & 9.94 & 2.73 & 9.96 & 1.91 & 0.51 & 10.25 & 2.79 & 2.01 & 0.69 & 3.59 & 9.36 \\
\hline UGC 6460 & 10.16 & 2.74 & 9.51 & 1.12 & 0.18 & 10.25 & 2.75 & 2.26 & 0.84 & 2.78 & 4.40 \\
\hline UGC 6746 & 11.54 & 2.81 & 10.08 & 0.53 & 0.03 & 11.56 & 2.81 & 8.12 & 0.59 & 4.18 & 14.81 \\
\hline UGC 7169 & 10.23 & 2.95 & 10.05 & 1.90 & 0.40 & 10.45 & 2.99 & 2.02 & 0.83 & 2.53 & 3.71 \\
\hline UGC 7315 & 10.06 & 3.26 & 8.88 & 1.15 & 0.06 & 10.09 & 3.26 & 1.00 & 0.88 & 2.32 & 7.13 \\
\hline UGC 7523 & 10.32 & 2.66 & 9.13 & 0.65 & 0.06 & 10.35 & 2.66 & 2.41 & 0.81 & 2.21 & 6.07 \\
\hline UGC 7901 & 10.22 & 3.16 & 9.79 & 1.84 & 0.27 & 10.36 & 3.18 & 1.46 & 0.63 & 4.28 & 9.06 \\
\hline UGC 8279 & 10.44 & 2.87 & 10.09 & 1.65 & 0.31 & 10.60 & 2.90 & 2.67 & 0.80 & 2.65 & 5.95 \\
\hline UGC 8865 & 10.63 & 2.26 & 9.77 & 0.58 & 0.12 & 10.69 & 2.27 & 5.65 & 0.50 & 6.03 & 11.84 \\
\hline UGC 9481 & 10.65 & 2.39 & 10.57 & 1.45 & 0.45 & 10.91 & 2.44 & 6.49 & 0.85 & 2.41 & 6.65 \\
\hline UGC 9926 & 10.76 & 3.05 & 9.86 & 1.33 & 0.11 & 10.81 & 3.06 & 2.61 & 0.76 & 2.51 & 5.15 \\
\hline UGC 9943 & 10.65 & 2.92 & 9.99 & 1.42 & 0.18 & 10.73 & 2.94 & 2.86 & 0.80 & 2.48 & 4.87 \\
\hline UGC 10083 & 10.34 & 2.59 & 9.79 & 1.20 & 0.22 & 10.45 & 2.60 & 3.02 & 0.83 & 2.29 & 3.51 \\
\hline UGC 10445 & 9.59 & 2.36 & 9.56 & 1.40 & 0.48 & 9.87 & 2.41 & 2.13 & 0.70 & 3.88 & 8.99 \\
\hline UGC 11628 & 11.43 & 2.82 & 10.38 & 0.99 & 0.08 & 11.47 & 2.83 & 6.92 & 0.67 & 2.99 & 9.83 \\
\hline UGC 11872 & 10.48 & 3.40 & 9.89 & 2.05 & 0.20 & 10.58 & 3.42 & 1.28 & 0.89 & 1.73 & 4.99 \\
\hline UGC 12343 & 11.19 & 2.64 & 10.53 & 1.11 & 0.18 & 11.28 & 2.65 & 7.52 & 0.69 & 3.44 & 9.79 \\
\hline UGC 12511 & 10.30 & 2.28 & 10.63 & 1.83 & 0.68 & 10.80 & 2.41 & 5.89 & 0.58 & 5.15 & 19.15 \\
\hline UGC 12614 & 10.93 & 2.71 & 10.32 & 1.15 & 0.20 & 11.03 & 2.72 & 5.60 & 0.84 & 2.71 & 5.17 \\
\hline UGC 12638 & 10.93 & 2.28 & 10.42 & 0.88 & 0.24 & 11.05 & 2.30 & 8.98 & 0.58 & 5.17 & 13.91 \\
\hline UGC 12654 & 10.57 & 2.45 & 10.34 & 1.31 & 0.37 & 10.77 & 2.48 & 5.43 & 0.74 & 3.37 & 7.42 \\
\hline UGC 6399 & 9.11 & 1.78 & 9.17 & 0.88 & 0.54 & 9.44 & 1.83 & 2.57 & 0.49 & 7.99 & 21.95 \\
\hline UGC 6446 & 9.12 & 2.22 & 9.42 & 1.32 & 0.66 & 9.60 & 2.27 & 2.05 & 0.72 & 4.58 & 15.14 \\
\hline NGC 3726 & 10.26 & 2.60 & 10.22 & 1.57 & 0.48 & 10.54 & 2.64 & 3.63 & 0.77 & 3.35 & 5.51 \\
\hline NGC 3769 & 9.81 & 2.73 & 9.94 & 1.96 & 0.57 & 10.18 & 2.80 & 1.91 & 0.88 & 2.40 & 8.36 \\
\hline NGC 3877 & 10.52 & 2.92 & 9.60 & 1.09 & 0.11 & 10.57 & 2.93 & 2.55 & 0.90 & 2.24 & 4.12 \\
\hline NGC 3893 & 10.47 & 2.89 & 10.15 & 1.70 & 0.32 & 10.64 & 2.92 & 2.71 & 0.80 & 2.64 & 5.32 \\
\hline NGC 3917 & 10.09 & 2.36 & 9.57 & 0.91 & 0.23 & 10.20 & 2.37 & 3.21 & 0.64 & 4.47 & 7.42 \\
\hline NGC 3949 & 10.07 & 2.89 & 9.79 & 1.78 & 0.35 & 10.25 & 2.92 & 1.70 & 0.76 & 2.86 & 4.40 \\
\hline NGC 3953 & 10.98 & 2.96 & 9.90 & 0.99 & 0.08 & 11.01 & 2.96 & 3.95 & 0.87 & 2.23 & 5.83 \\
\hline NGC 3972 & 9.81 & 2.62 & 9.43 & 1.26 & 0.30 & 9.96 & 2.63 & 1.88 & 0.70 & 4.14 & 8.39 \\
\hline UGC 6917 & 9.64 & 2.01 & 9.40 & 0.83 & 0.37 & 9.84 & 2.04 & 3.13 & 0.53 & 6.65 & 13.62 \\
\hline UGC 6923 & 9.44 & 2.21 & 9.07 & 0.92 & 0.30 & 9.59 & 2.23 & 1.85 & 0.72 & 3.51 & 6.50 \\
\hline NGC 3992 & 10.82 & 2.80 & 10.25 & 1.27 & 0.21 & 10.93 & 2.81 & 4.56 & 0.64 & 4.72 & 8.98 \\
\hline UGC 6983 & 9.73 & 2.06 & 9.82 & 1.22 & 0.55 & 10.08 & 2.12 & 3.78 & 0.65 & 4.46 & 12.68 \\
\hline NGC 4051 & 10.22 & 2.79 & 9.75 & 1.35 & 0.26 & 10.34 & 2.81 & 2.38 & 0.82 & 2.98 & 4.47 \\
\hline NGC 4088 & 10.49 & 2.85 & 10.12 & 1.56 & 0.30 & 10.64 & 2.87 & 2.99 & 0.84 & 2.59 & 5.32 \\
\hline
\end{tabular}


Table 2. Continued.

\begin{tabular}{cccccccccccc}
\hline \hline Name & $\operatorname{Lg} M_{\mathrm{s}}{ }^{a}$ & $\operatorname{Lg} \Sigma_{\mathrm{s}, 0}{ }^{b}$ & $\operatorname{Lg} M_{\mathrm{g}}{ }^{c}$ & $\operatorname{Lg} \Sigma_{g}^{d}$ & $f_{g}^{e}$ & $\operatorname{Lg} M_{\mathrm{bar}}{ }^{f}$ & $\operatorname{Lg} \Sigma_{\mathrm{d}, 0}{ }^{g}$ & $h_{\mathrm{d}}{ }^{h}$ & $\left(V_{\mathrm{d}, \mathrm{m}} / V_{\mathrm{t}, \mathrm{m}}\right)^{i}$ & $M_{\mathrm{dyn}} / M_{\mathrm{bar}}{ }^{j}$ & $M_{\mathrm{dyn}} / L_{B}{ }^{k}$ \\
\hline NGC 4100 & 10.53 & 2.92 & 10.12 & 1.55 & 0.28 & 10.67 & 2.93 & 2.93 & 0.84 & 2.73 & 5.17 \\
NGC 4102 & 10.55 & 3.34 & 9.32 & 1.31 & 0.06 & 10.58 & 3.34 & 1.39 & 0.91 & 1.67 & 6.76 \\
NGC 3718 & 10.64 & 2.33 & 10.01 & 0.89 & 0.19 & 10.73 & 2.35 & 5.42 & 0.47 & 6.67 & 11.35 \\
NGC 3729 & 10.31 & 3.11 & 9.29 & 1.11 & 0.09 & 10.35 & 3.11 & 1.69 & 1.02 & 1.91 & 5.50 \\
UGC 6773 & 9.01 & 1.90 & 8.70 & 0.70 & 0.33 & 9.19 & 1.93 & 1.62 & 0.68 & 3.85 & 7.09 \\
UGC 6818 & 9.05 & 1.97 & 9.13 & 1.08 & 0.55 & 9.39 & 2.03 & 1.94 & 0.61 & 5.26 & 14.72 \\
NGC 3985 & 9.44 & 2.66 & 8.98 & 1.21 & 0.26 & 9.57 & 2.67 & 1.15 & 0.83 & 2.95 & 3.81 \\
UGC 7089 & 9.28 & 1.82 & 9.40 & 0.92 & 0.57 & 9.65 & 1.87 & 3.18 & 0.72 & 4.00 & 11.50 \\
UGC 7094 & 8.74 & 1.93 & 8.92 & 1.08 & 0.60 & 9.14 & 1.99 & 1.55 & 1.05 & 1.91 & 6.32 \\
NGC 4117 & 9.53 & 2.22 & 9.12 & 1.22 & 0.28 & 9.67 & 2.26 & 1.55 & 0.38 & 7.80 & 22.07 \\
NGC 4138 & 10.40 & 3.28 & 8.85 & 0.94 & 0.03 & 10.41 & 3.28 & 1.22 & 0.84 & 1.94 & 7.04 \\
NGC 4218 & 8.97 & 2.74 & 8.63 & 1.46 & 0.32 & 9.13 & 2.76 & 0.61 & 0.80 & 2.92 & 3.42 \\
NGC 4220 & 10.30 & 3.16 & 9.13 & 1.10 & 0.06 & 10.32 & 3.17 & 1.43 & 0.74 & 3.15 & 5.39 \\
UGC 128 & 10.05 & 1.62 & 10.16 & 0.73 & 0.56 & 10.41 & 1.67 & 9.57 & 0.41 & 12.24 & 35.99 \\
F563-V2 & 9.54 & 2.11 & 9.60 & 1.18 & 0.54 & 9.87 & 2.16 & 2.93 & 0.87 & 2.66 & 7.34 \\
F568-3 & 9.85 & 1.96 & 9.82 & 0.95 & 0.49 & 10.14 & 2.00 & 4.75 & 0.59 & 5.87 & 14.11 \\
F574-1 & 9.86 & 1.65 & 9.83 & 0.82 & 0.49 & 10.15 & 1.71 & 6.07 & 0.58 & 4.87 & 12.36 \\
F583-1 & 9.11 & 1.67 & 9.52 & 1.43 & 0.72 & 9.67 & 1.87 & 2.94 & 0.55 & 5.56 & 26.16 \\
MilkyWay & 10.64 & 2.82 & 10.09 & 1.38 & 0.22 & 10.75 & 2.83 & 3.40 & 0.71 & 3.42 & 10.50 \\
Andromeda & 11.09 & 3.07 & 10.05 & 1.08 & 0.08 & 11.13 & 3.07 & 4.26 & 0.87 & 2.54 & 6.83 \\
\hline
\end{tabular}

a Total stellar mass (disk+bulge) in $M_{\odot}$.

${ }^{b}$ Disk stellar central surface density in $M_{\odot} \mathrm{pc}^{-2}$.

c Total gas mass in $M_{\odot}$

${ }^{d}$ Disk gas central surface density in $M_{\odot} \mathrm{pc}^{-2}$.

$e$ Gas fraction $\left(M_{\mathrm{g}} /\left[M_{\mathrm{g}}+M_{\mathrm{s}}\right]\right)$.

${ }^{f}$ Total baryonic mass $\left(M_{\mathrm{s}}+M_{\mathrm{g}}\right)$ in $M_{\odot}$.

$g$ Disk total central surface density in $M_{\odot} \mathrm{pc}^{-2}$.

${ }^{h}$ Disk (stellar + gaseous) scale radius in kpc.

${ }^{i}$ Disk-to-total maximum velocity ratio.

$j$ Dynamical-to-baryonic mass ratio at $5 h_{\mathrm{d}}$.

${ }^{k}$ Dynamical mass-to-light ratio in the band $B$ at $5 h_{\mathrm{d}}\left(M_{\odot} / L_{B \odot}\right)$.

the observational results with theoretical predictions. As mentioned in the introduction, the analysis of the rotation curve shapes offers a powerful method to estimate the fraction of luminous and dark matter in galaxies at a given radius $\left(3.2 h_{\mathrm{d}}\right.$, for example). The approach used here is conceptually different. In our sample, we have information only about the HI velocity line-width, $W_{20}$, which approximately corresponds to twice the maximum total rotation velocity $V_{\mathrm{t}, \mathrm{m}}$ (see introduction), but not about the rotation curve shapes. On the other hand, with the information about the disk structure that we have (assuming exponential stellar and gas distributions), the velocity component $V_{\mathrm{d}}$ due to the total disk can be calculated (Eq. (5) below), for example at the radius where $V_{\mathrm{d}}$ attains its maximum, $2.2 h_{\mathrm{d}}$. Thus, without introducing further assumptions, we may define the ratio of disk maximum velocity to total (or dynamical) maximum velocity, $\left(V_{\mathrm{d}, \mathrm{m}} / V_{\mathrm{t}, \mathrm{m}}\right)$, which can be directly compared with theoretical predictions.

The $\left(V_{\mathrm{d}, \mathrm{m}} / V_{\mathrm{t}, \mathrm{m}}\right)$ ratio is not defined at a given radius but it can be related to the dynamical-to-baryon (disk) mass ratio, $M_{\mathrm{dyn}} / M_{\mathrm{bar}}$, defined at the radius where the total rotation curve peaks, $r_{\text {peak }}$. Assuming an exponential disk $\left(M_{\text {bar }} \propto \Sigma_{\mathrm{d}, 0} h_{\mathrm{d}}{ }^{2}\right)$, one obtains:

$\left(\frac{M_{\mathrm{dyn}}}{M_{\mathrm{bar}}}\right)_{\text {peak }} \approx \frac{V_{\mathrm{t}, \mathrm{m}}{ }^{2} r_{\text {peak }} / G}{f_{\mathrm{L}} 2 \pi \Sigma_{\mathrm{d}, 0} h_{\mathrm{d}}{ }^{2}} \propto \frac{V_{\mathrm{t}, \mathrm{m}}^{2} x h_{\mathrm{d}}}{f_{\mathrm{L}}(x) \Sigma_{\mathrm{d}, 0} h_{\mathrm{d}}^{2}} \propto \frac{x}{f_{\mathrm{L}}(x)}\left(\frac{V_{\mathrm{d}, \mathrm{m}}}{V_{\mathrm{t}, \mathrm{m}}}\right)^{-2}$,

where $r_{\text {peak }}=x h_{\mathrm{d}}, f_{\mathrm{L}}$ is the fraction of the total disk mass at $r_{\text {peak }}$, and $V_{\mathrm{d}, \mathrm{m}}$ is calculated according to Eq. (5) below. So, in order to calculate $M_{\mathrm{dyn}} / M_{\mathrm{bar}}$, one needs to define the coefficients $x$ and $f_{\mathrm{L}}(x)$; they actually may change from galaxy to galaxy, depending on the disk SB, luminosity, and halo density profile $\left(x \approx 2.2\right.$ and $f_{\mathrm{L}} \approx 0.64$ for disk dominated galaxies, and probably, $x>2.2$ and $f_{\mathrm{L}}>0.64$ for halo dominated galaxies). Previous works, based on the analysis of rotation curves, have shown that $M_{\mathrm{dyn}} / M_{\mathrm{bar}}$ (at $3.2 h_{\mathrm{d}}$ ) has a strong dependence on luminosity (Persic \& Salucci 1988, 1990a; Persic et al. 1996). In Sect. 4 we will present results for $M_{\text {dyn }} / M_{\text {bar }}$. Here we concentrate on $\left(V_{\mathrm{d}, \mathrm{m}} / V_{\mathrm{t}, \mathrm{m}}\right)$, which is more directly comparable to model results than $M_{\text {dyn }} / M_{\text {bar }}$, and may offer a first idea of how the fractions of dark and luminous matter in galaxies depend on several galaxy properties. 
In order to calculate $V_{\mathrm{d}, \mathrm{m}}$ from the observations, we use the expression of $V_{\mathrm{d}}$ for a flattened exponential disk (Freeman 1970):

$V_{\mathrm{d}}^{2}(y)=4 \pi G \Sigma_{\mathrm{d}, 0} h_{\mathrm{d}} y^{2} I(y)$,

where $y=r /\left(2 h_{\mathrm{d}}\right), G$ is the gravitational constant, and $I(y)$ is a function composed of modified Bessel functions of the first and second kinds. The function (5) has its maximum at 2.2 $h_{\mathrm{d}}$. Notice that $\Sigma_{\mathrm{d}, 0}$ and $h_{\mathrm{d}}$ are not directly observed quantities (see Sect. 2.2). Although the assumptions made to calculate them from the observational data are robust, we will check a posteriori whether our results are significantly dependent on these assumptions.

According to Sacket (1997, and more references therein), the maximum disk hypothesis applied to individual $\mathrm{Sb}-\mathrm{Sc}$ galaxies implies a baryonic disk mass (including gas) which provides $(85 \pm 10) \%$ of the total rotation support at $2.2 h_{\mathrm{d}}$ $\left(2.2 h_{K}\right)$. The lower limit is occupied by galaxies with large bulge; only in these cases the bulge contribution to $V_{\mathrm{t}, \mathrm{m}}$ may become significant. From the analysis of rotation curve shapes and assuming the maximum disk hypothesis, Persic \& Salucci (1990b) reported $M_{\mathrm{bar}} / M_{\mathrm{dyn}}$ ratios at $3.2 h_{\mathrm{d}}$ increasing with $L_{B}$ from 0.3 to 0.8 . This implies that $\left(V_{\mathrm{d}} / V_{\mathrm{t}}\right)_{3.2 h_{\mathrm{d}}}$ oscillates between 0.57 and 0.91 . The $V_{\mathrm{d}} / V_{\mathrm{t}}$ ratio increases from 3.2 to $2.2 h_{\mathrm{d}}$; by using the rotation curve decompositions in Fig. 3 of Persic et al. (1996), we estimate that $\left(V_{\mathrm{d}} / V_{\mathrm{t}}\right)_{2.2 h_{\mathrm{d}}}$ would oscillate between 0.75 and 0.95 , in excellent agreement with Sacket (1997). Although $\left(V_{\mathrm{d}, \mathrm{m}} / V_{\mathrm{t}, \mathrm{m}}\right)$ is not formally equal to $\left(V_{\mathrm{d}} / V_{\mathrm{t}}\right)_{2.2 h_{\mathrm{d}}}$, as a reference we will say that the maximum hypothesis implies $\left(V_{\mathrm{d}, \mathrm{m}} / V_{\mathrm{t}, \mathrm{m}}\right) \approx 0.85 \pm 0.1$. Since the individual rotation curve decomposition methods usually take into account the disk thickness $(q>0)$, we will correct $V_{\mathrm{d}, \mathrm{m}}$ as given in Eq. (5) for disk thickness. Assuming a minor-to-major disk axis ratio $q$ of $0.2, V_{\mathrm{d}, \mathrm{m}}$ becomes approximately $5 \%$ lower than for the infinitely thin disk case (e.g., Burlak et al. 1997). We will apply this correction factor to the disk+halo composite mass models presented below as well to the observational data.

Before studying and comparing the inferred $\left(V_{\mathrm{d}, \mathrm{m}} / V_{\mathrm{t}, \mathrm{m}}\right)$ ratios with results from galaxy evolution models, we shall explore how this ratio is expected to depend on different disk and halo parameters. To this purpose, simple disk+halo composite mass models will be used, and then they will be compared to the complex evolutionary models.

\subsection{Composite mass models using analytical halo profiles}

Here we will analyze the rotation curve decomposition corresponding to exponential disks in centrifugal equilibrium inside pseudo-isothermal (e.g., de Blok \& McGaugh 1997) and Navarro et al. (1997; hereafter NFW) dark halos. A description of how the halos and disks are constructed is given in Appendix A. In Fig. 2a we present $\left(V_{\mathrm{d}, \mathrm{m}} / V_{\mathrm{t}, \mathrm{m}}\right)$ vs. the central disk surface density, $\Sigma_{\mathrm{d}, 0}$, for the virial (halo) mass $M_{\mathrm{v}}=$ $3.5 \times 10^{11} M_{\odot}$ and for the two halo models. Upper and lower curves correspond to the pseudo-isothermal and cosmological NFW halos, respectively. In the former case, the assumed core radius was $3.8 \mathrm{kpc}$ (see Appendix A). The solid lines are for the global baryon-to-halo mass ratio $f_{\mathrm{d}}=0.05$, while dashed

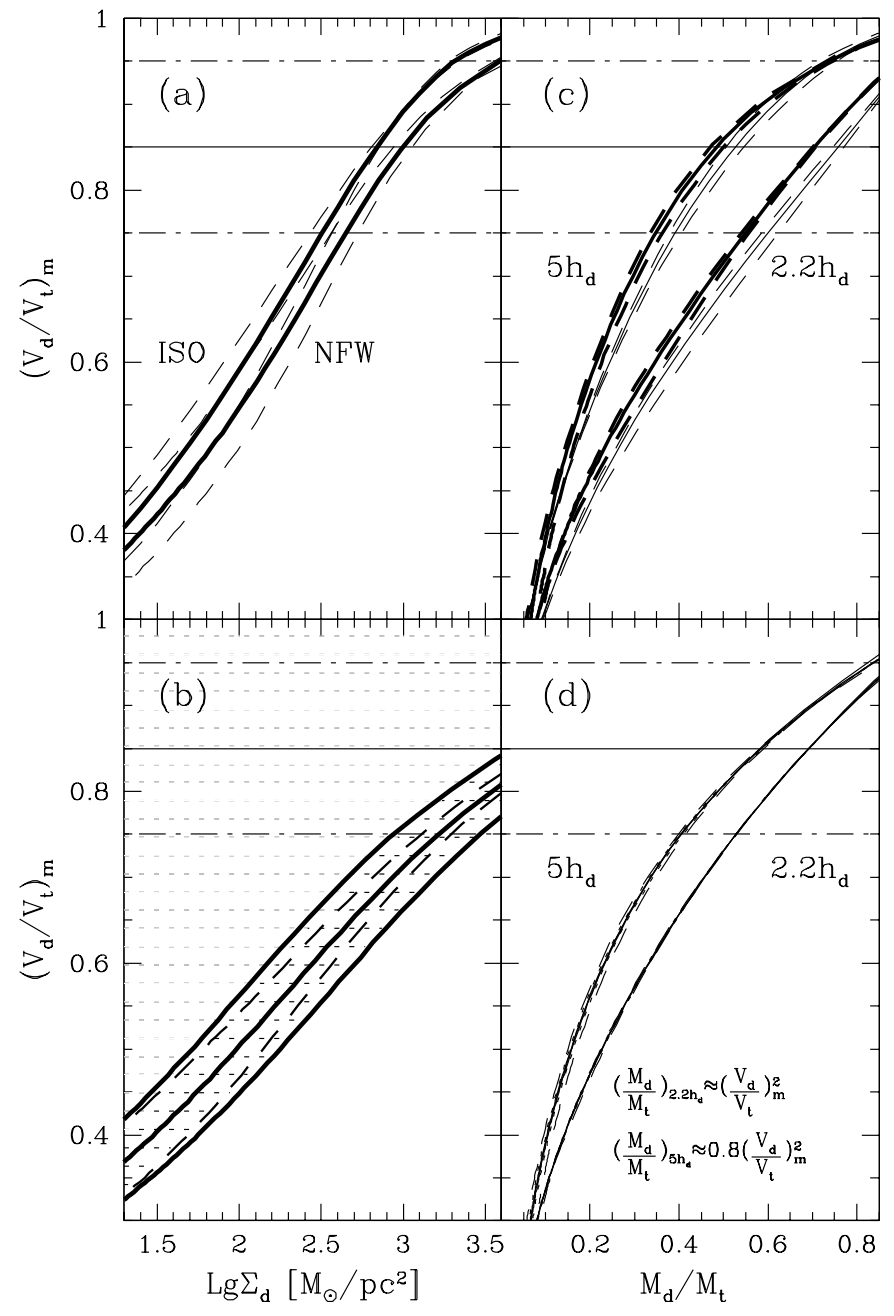

Fig. 2. a). Correlation between $\left(V_{\mathrm{d}, \mathrm{m}} / V_{\mathrm{t}, \mathrm{m}}\right)$ and $\Sigma_{\mathrm{d}, 0}$ for disks in centrifugal equilibrium inside a pseudo-isothermal (upper curves) and a NFW (lower curves) halo of $M_{\mathrm{v}}=3.5 \times 10^{11} M_{\odot}$. See details about the model parameters in the text. Solid lines are for $f_{\mathrm{d}}=0.05$, while upper and lower dashed lines around the solid curves are for $f_{\mathrm{d}}=0.08$ and 0.03 , respectively. The approximate region of maximum disk (see text) is bounded by the dot-dashed lines. b) Same as in panel a), but for the NFW model, taking into account the disk gravitational dragging over the halo. The shaded region encompasses the $\pm 1 \sigma$ scatter in concentrations around the central model. The solid upper and lower curves are for $M_{\mathrm{v}}=3.5 \times 10^{12} M_{\odot}$ and $3.5 \times 10^{10} M_{\odot}$ with the corresponding average concentration parameters and $f_{\mathrm{d}}=0.05$. c) and d). Relation between $\left(V_{\mathrm{d}, \mathrm{m}} / V_{\mathrm{t}, \mathrm{m}}\right)$ and the disk(baryon)-to-dynamical mass ratios inside $2.2 h_{\mathrm{d}}$ and $5 h_{\mathrm{d}}$ for the same models shown in the left panels. In panel c) thick and thin lines are for the NFW and pseudoisothermal halos, respectively. In panel d) the linear fittings (in LogLog) are given.

lines are for $f_{\mathrm{d}}=0.08$ (upper) and 0.03 (lower). For the NFW halos, we have used the average concentration parameter for a given mass taken from Eke et al. (2001). The $\Lambda$ CDM cosmology with $\Omega_{m}=0.3, \Omega_{\Lambda}=0.7, h=0.7$ is used here. The disk central surface density is determined mainly by the halo spin parameter $\lambda$, but depends also on $M_{\mathrm{v}}$ and $f_{\mathrm{d}}$ (see Appendix A). Figure 2 is for a range of $\lambda$ from 0.015 to 0.2 . The trend of decreasing $\left(V_{\mathrm{d}, \mathrm{m}} / V_{\mathrm{t}, \mathrm{m}}\right)$ as $\Sigma_{\mathrm{d}, 0}$ decreases is clearly seen for both halo models. Obviously, for the pseudo-isothermal halos, the 
disk velocity component dominates more than for the cuspy NFW halos.

While the pseudo-isothermal halo profile is an adhoc model introduced to fit some observed rotation curves, the NFW profile is a theoretical prediction for the structure of the halos in the CDM cosmogony. In the latter case, it is important to take into account that the inner structure of these halos is affected by the formation of the baryonic galaxy, which gravitationally contracts the original halo. The usual way to calculate this effect is by assuming a radial adiabatic invariance during the formation of the disk (e.g., Flores et al. 1993; Mo et al. 1998; see Appendix A). In Fig. 2b, the same curves for the NFW model presented in panel a are shown, but this time taking into account the halo contraction due to disk formation. Two more masses, $M_{\mathrm{v}}=3.5 \times 10^{10} M_{\odot}$ and $3.5 \times 10^{12} M_{\odot}$ are added in this panel. As one sees, when the halo model is originally of NFW type, after disk formation, even disks with high surface densities tend to be of sub-maximal type: $\left(V_{\mathrm{d}, \mathrm{m}} / V_{\mathrm{t}, \mathrm{m}}\right)$ almost never exceeds $\sim 0.75-0.80^{3}$. The effects of the halo contraction on the $\left(V_{\mathrm{d}, \mathrm{m}} / V_{\mathrm{t}, \mathrm{m}}\right)$ ratio are non-significant only for the lowest surface density disks. For the $M_{\mathrm{v}}=3.5 \times 10^{11} M_{\odot}$ model with $f_{\mathrm{d}}=0.05$, we also show the scatter produced in the $\left(V_{\mathrm{d}, \mathrm{m}} / V_{\mathrm{t}, \mathrm{m}}\right)$ $\Sigma_{\mathrm{d}, 0}$ diagram by the different halo concentrations. The shaded region encompasses $\pm 1 \sigma$ deviations in $c\left(\Delta \log c_{\mathrm{NFW}} \approx 0.18\right.$, Bullock et al. 2001; the lower limit corresponding to the $+1 \sigma$ value).

A comparison in the $\left(V_{\mathrm{d}, \mathrm{m}} / V_{\mathrm{t}, \mathrm{m}}\right)-\Sigma_{\mathrm{d}, 0}$ plane of the pseudoisothermal and NFW halos (taking into account the disk gravitational drag in the last case), shows that as $\Sigma_{\mathrm{d}, 0}$ is higher, the $\left(V_{\mathrm{d}, \mathrm{m}} / V_{\mathrm{t}, \mathrm{m}}\right)$ ratio for the pseudo-isothermal models becomes larger than that for the contracted NFW models. Thus, it is at high surface densities (SBS) where the differences in the $\left(V_{\mathrm{d}, \mathrm{m}} / V_{\mathrm{t}, \mathrm{m}}\right)$ ratio are more sensitive to whether the halo is cuspy or shallow. On the other hand, neither the concentration nor the mass introduce significant differences in $\left(V_{\mathrm{d}, \mathrm{m}} / V_{\mathrm{t}, \mathrm{m}}\right)$.

For the NFW models with $M_{\mathrm{v}}=3.5 \times 10^{10} M_{\odot}$ (upper thin solid line) and $3.5 \times 10^{12} M_{\odot}$ (lower thin solid line), we also have used the average NFW concentrations corresponding to these masses (Eke et al. 2001) and $f_{\mathrm{d}}=0.05$. Massive systems result with lower $\left(V_{\mathrm{d}, \mathrm{m}} / V_{\mathrm{t}, \mathrm{m}}\right)$ ratios than the less massive ones, which apparently would imply that the former are more dark matter dominated than the latter. In fact, the average concentration for less massive halos is higher than for the more massive ones. However, it is important to have in mind that the parameters of the modeled disks are connected to the scale of the halo: $h_{\mathrm{d}}$ and $\Sigma_{\mathrm{d}, 0}$ depend on $M_{\mathrm{v}}$ (see Appendix A). For a given $\lambda$ (constant $\Sigma_{\mathrm{d}, 0}$ ), $h_{\mathrm{d}} \propto V_{\mathrm{t}, \mathrm{m}}{ }^{\beta}$, with $\beta \approx 1.5$, i.e. shallower than 2 (Firmani \& Avila-Reese 2000, hereafter FA00). For constant $\Sigma_{\mathrm{d}, 0},\left(V_{\mathrm{d}, \mathrm{m}} / V_{\mathrm{t}, \mathrm{m}}\right) \propto h_{\mathrm{d}}^{0.5} / V_{\mathrm{t}, \mathrm{m}}$. Therefore, $\left(V_{\mathrm{d}, \mathrm{m}} / V_{\mathrm{t}, \mathrm{m}}\right) \propto$ $V_{\mathrm{t}, \mathrm{m}}{ }^{-0.25}$, i.e. $\left(V_{\mathrm{d}, \mathrm{m}} / V_{\mathrm{t}, \mathrm{m}}\right)$ decreases with increasing $V_{\mathrm{t}, \mathrm{m}}\left(\right.$ or $\left.M_{\mathrm{v}}\right)$. This effect is partially compensated by the fact that more massive halos are less concentrated (lower $\left(V_{\mathrm{d}, \mathrm{m}} / V_{\mathrm{t}, \mathrm{m}}\right)$ ). The final result is a small dependence of $\left(V_{\mathrm{d}, \mathrm{m}} / V_{\mathrm{t}, \mathrm{m}}\right)$ on mass. For a

\footnotetext{
${ }^{3}$ As mentioned above, the $V_{\mathrm{d}, \mathrm{m}}$ calculated using Eq. (5) is lowered by $5 \%$ in order to take into account for the disk thickness $(q=0.2)$. This correction enters also in the total rotation velocity because this last is the sum in quadrature of the disk and halo velocity components.
}

variation of 2 orders of magnitude in mass, the change in $\left(V_{\mathrm{d}, \mathrm{m}} / V_{\mathrm{t}, \mathrm{m}}\right)$ is around $16 \%$ for a medium value of $\Sigma_{\mathrm{d}, 0}=$ $400 M_{\odot} / \mathrm{pc}^{2}$ (Fig. 2b). The change of $\left(V_{\mathrm{d}, \mathrm{m}} / V_{\mathrm{t}, \mathrm{m}}\right)$ for a $\pm 1 \sigma$ in concentration is close to $12 \%$ for the same central surface density.

The dependence of the $\left(V_{\mathrm{d}, \mathrm{m}} / V_{\mathrm{t}, \mathrm{m}}\right)$ ratio on $f_{\mathrm{d}}$ is also small. Curves with dashed lines in Fig. $2 b$ are for the same central model $\left(M_{\mathrm{v}}=3.5 \times 10^{11} M_{\odot}\right.$ and average concentration parameter) but $f_{\mathrm{d}}$ from 0.08 (upper curve) to $f_{\mathrm{d}}=0.03$ (lower curve). The $\left(V_{\mathrm{d}, \mathrm{m}} / V_{\mathrm{t}, \mathrm{m}}\right)$ ratio does not change more than $10 \%$ within this range of $f_{\mathrm{d}}$ for $\Sigma_{\mathrm{d}, 0}$ around $400 M_{\odot} / \mathrm{pc}^{2}$.

The $\left(V_{\mathrm{d}, \mathrm{m}} / V_{\mathrm{t}, \mathrm{m}}\right)$ ratio is directly related to the disk and halo mass fractions. In order to gain a more quantitative feeling of the $\left(V_{\mathrm{d}, \mathrm{m}} / V_{\mathrm{t}, \mathrm{m}}\right)$ ratio, Fig. 2, panels $\mathrm{c}$ and $\mathrm{d}$ show the corresponding disk-to-dynamical mass ratios at $2.2 h_{\mathrm{d}}$ and $5 h_{\mathrm{d}}$ for the same curves shown in panels a and $b$. When these ratios are 0.5 , the disk and the halo masses are equal at $2.2 h_{\mathrm{d}}$ and $5 h_{\mathrm{d}}$, respectively. The rotation curve peaks typically at $2.2 h_{\mathrm{d}}$, while $5 h_{\mathrm{d}}$ corresponds approximately to the optical disk radius. For the gravitationaly contracted NFW halo model (panel d), the maximum disk case $\left(\left(V_{\mathrm{d}, \mathrm{m}} / V_{\mathrm{t}, \mathrm{m}}\right)>0.75\right)$ implies that $53 \%$ or more of the dynamical mass at $2.2 h_{\mathrm{d}}$ should be in the disk. At $5 h_{\mathrm{d}}$ this lower limit decreases down to $\approx 40 \%$. Such high values can be attained only for ultra-high surface density disks. It is interesting to note that, for the NFW halos with contraction and for a large range of disk masses and surface densities, $\left(V_{\mathrm{d}, \mathrm{m}} / V_{\mathrm{t}, \mathrm{m}}\right) \approx\left(M_{\mathrm{dyn}} / M_{\mathrm{bar}}\right)_{2.2 h_{\mathrm{d}}}^{-1 / 2}=\left(V_{\mathrm{d}} / V_{\mathrm{t}}\right)_{2.2 h_{\mathrm{d}}}$.

\subsection{Self-consistent galaxy evolution models}

In previous papers (Avila-Reese et al. 1998; FA00; Avila-Reese \& Firmani 2000; see also van den Bosch 1998, 2000, 2002) semi-numerical models of disk galaxy formation and evolution in the hierarchical $\Lambda \mathrm{CDM}$ scenario were developed. These models include self-consistently halo formation and evolution, disk star formation (SF) and feedback processes, the gravitational dragging of the halo due to disk formation, secular bulge formation and other evolution processes. The initial and border conditions of the models are $M_{\mathrm{v}}, f_{\mathrm{d}}$, the halo mass aggregation history (MAH) and the spin parameter $\lambda$. A short description of the main ingredients of the models is presented in Appendix B.

Before analyzing the predictions of these models regarding the $\left(V_{\mathrm{d}, \mathrm{m}} / V_{\mathrm{t}, \mathrm{m}}\right)$ ratio, it is important to note the following three effects. (i) The halo contraction due to disk formation in the models was calculated for elliptical orbits, i.e. the adiabatic invariance was not limited to only radial orbits, as was done in the previous subsection. Particles within the virialized $\Lambda \mathrm{CDM}$ halos have orbits with ratios of apapsis-to-periapsis around 0.15-0.40 (Ghigna et al. 1998). Therefore, the adiabatic halo contraction for the evolutionary models presented here is of lower amplitude than in the case of assuming radial orbits (previous subsection). (ii) The disks formed within $\Lambda \mathrm{CDM}$ halos, assuming detailed angular momentum conservation, have actually some excess in the center (cusp) w.r.t. the exponential surface density distribution (FA00; Avila-Reese \& Firmani 2002; see also Bullock et al. 2001; van den Bosch 2002), which give rise to a bulge formed secularly (Avila-Reese \& Firmani 2000). The effects (i) and (ii) imply that $V_{\mathrm{d}, \mathrm{m}}$ and the $\left(V_{\mathrm{d}, \mathrm{m}} / V_{\mathrm{t}, \mathrm{m}}\right)$ ratio measured in our evolutionary models should be larger than 
in the simple composite mass models, where the orbits were assumed radial and the disk surface density exponential (see previous subsection). (iii) Our model disks are not infinitely thin; they have a vertical density and velocity structure determined by the SF, feedback and infall mechanisms included in the modelation. The gravitational potential of the disk is calculated taking into account its three-dimensional structure. A comparison of the $V_{\mathrm{d}, \mathrm{m}}$ measured in the model galaxies with the $V_{\mathrm{d}, \mathrm{m}}$ calculated according to Eq. (5) shows that the former is on average $\sim 7 \%$ smaller than the latter. In fact we have corrected (uniformly) the composite mass models (and the observational data) to take into account the disk thickness. The $5 \%$ correction in $V_{\mathrm{d}, \mathrm{m}}$ calculated according to Burlak et al. (1997) is close to the $7 \%$ measured on average in the evolutionary models.

In Fig. 3a we present the results for 63 models grouped around three halo masses, $M_{\mathrm{v}} \approx 3.5 \times 10^{10}, 3.5 \times 10^{11}$, and $3.5 \times 10^{12} M_{\odot}$ (triangles, squares and circles, respectively) with MAHs and $\lambda$ 's obtained randomly from their corresponding (cosmological) statistical distributions, and $f_{\mathrm{d}}=0.05$ (FA00; Appendix B). The solid curves are the same curves plotted in Fig. $3 \mathrm{~b}$ for the three masses, the average concentration parameters, and $f_{\mathrm{d}}=0.05$. The evolutionary models seem to lie slightly higher than the corresponding composite mass models in the $\left(V_{\mathrm{d}, \mathrm{m}} / V_{\mathrm{t}, \mathrm{m}}\right)$ vs. $\Sigma_{\mathrm{d}, 0}$ plane, in particular at ultra-high surface densities, where the disk cusps are more pronounced. Although an attempt to introduce a correction due to disk thickness has been done for the composite mass models, it is important to keep in mind the other two effects mentioned above as well several evolution processes included in the modelation. Nevertheless, the simple disk +halo composite mass models offer a reasonable description of results from the evolutionary models and deserve to be included. These models can be easily reproduced by other workers in the field interested in using these kind of theoretical results.

The model $\left(V_{\mathrm{d}, \mathrm{m}} / V_{\mathrm{t}, \mathrm{m}}\right)$ ratio is determined mainly by the spin parameter $\lambda$ (FA00), therefore $\left(V_{\mathrm{d}, \mathrm{m}} / V_{\mathrm{t}, \mathrm{m}}\right)$ correlates mainly with $\Sigma_{\mathrm{d}, 0}$ as seen in Fig. 3a. The models show also a slight dependence of the $\left(V_{\mathrm{d}, \mathrm{m}} / V_{\mathrm{t}, \mathrm{m}}\right)$ ratio on mass (see the segregation of triangles, squares and circles in Fig. 3a). The reason of this dependence has been already explained in the previous subsection. For a given mass, the scatter seen in Fig. 3a is due to the scatter in the halo structures. This scatter has its origin in the dispersion of the MAHs (Avila-Reese et al. 1998; Wechsler et al. 2002). For perfect NFW halos, this scatter is completely described by the scatter in the NFW concentrations. Thus, more concentrated halos and more massive galaxies tend to have lower $\left(V_{\mathrm{d}, \mathrm{m}} / V_{\mathrm{t}, \mathrm{m}}\right)$ ratios for a given $\Sigma_{\mathrm{d}, 0}$. The differences are actually small.

\subsection{Observational data}

In Fig. $3 \mathrm{a}$ we plot the $\left(V_{\mathrm{d}, \mathrm{m}} / V_{\mathrm{t}, \mathrm{m}}\right)$ ratio vs. $\Sigma_{\mathrm{d}, 0}$ inferred from the compiled observations in Sect. 2. The scatter is large and before comparing to models, we shall explore whether the observed correlation is significant and direct. In Fig. 3a, four galaxies have been identified as outliers (shown with their names in the plots). These galaxies were excluded from any statistical analysis.

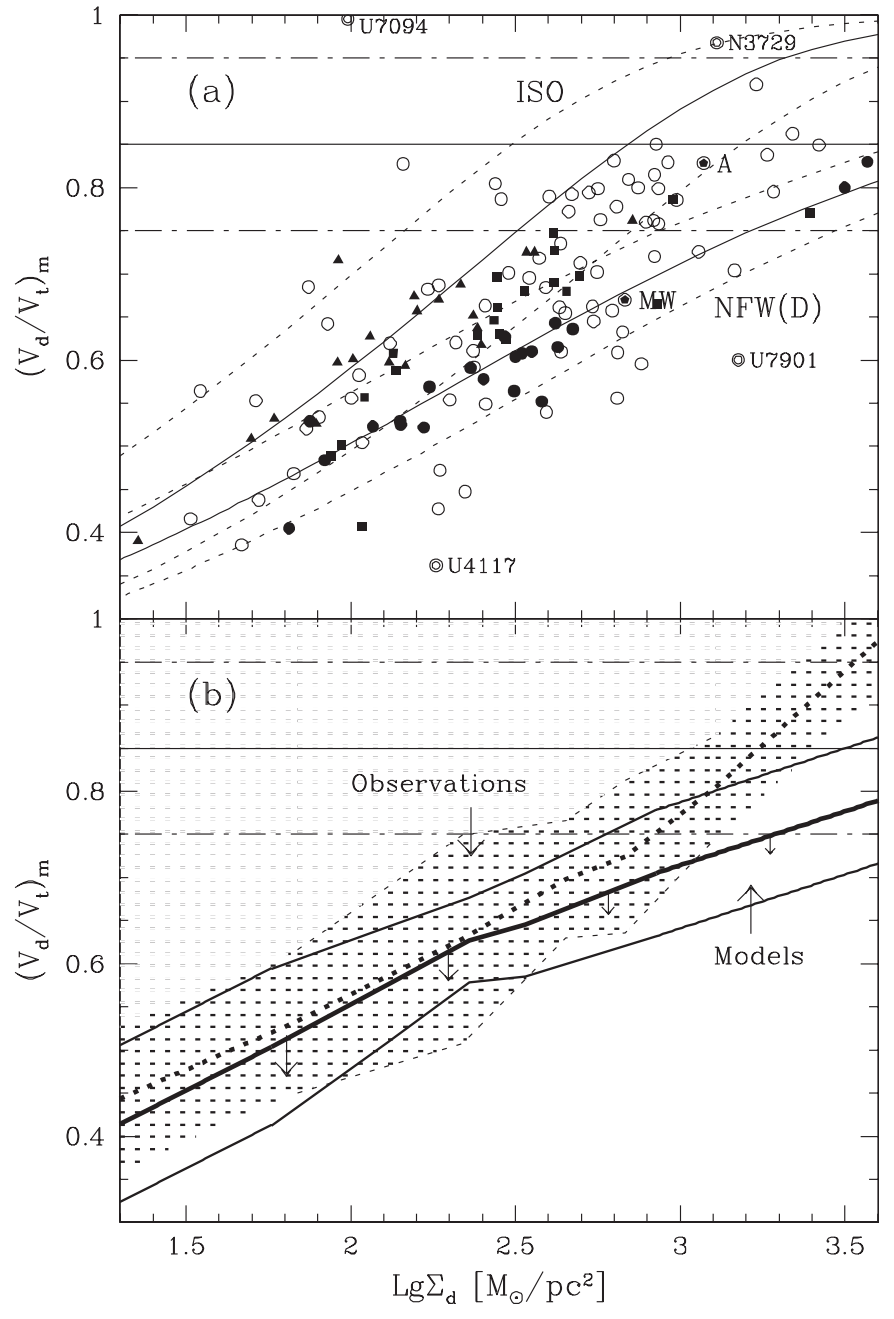

Fig. 3. a) Same as in Fig. 2 but for observations (empty circles) and evolutionary model results (solid triangles, squares, and circles corresponding to $M_{\mathrm{v}}=3.5 \times 10^{10} M_{\odot}, 3.5 \times 10^{11} M_{\odot}$, and $3.5 \times 10^{12} M_{\odot}$, respectively). The models are for $f_{\mathrm{d}}=0.05$. Four galaxies, which are outliers in this plot, as well as the Milky Way and Andromeda galaxies are indicated by their names. For indication, the composite mass models presented in Fig. 2 for the pseudo-isothermal halo (upper curves) and the NFW halo with gravitational drag (lower curves) are also shown. The solid lines are for $M_{\mathrm{v}}=3.5 \times 10^{11} M_{\odot}$ while the dashed ones are for $3.5 \times 10^{10} M_{\odot}$ (upper curves) and $3.5 \times 10^{12} M_{\odot}$ (lower curves). b) Means per $\Sigma_{\mathrm{d}, 0}$ bins and their corresponding standard deviations for the observational data (thick dashed line and shaded area) and the model results (thick solid line and empty frame) showed in panel a). The arrows from the thick curve are an estimate of how much the models would decrease if $f_{\mathrm{d}}$ is decreased to 0.03 .

In a first exploratory phase, we look for any correlation of the $\left(V_{\mathrm{d}, \mathrm{m}} / V_{\mathrm{t}, \mathrm{m}}\right)$ ratio $\left(\log \left(V_{\mathrm{d}, \mathrm{m}} / V_{\mathrm{t}, \mathrm{m}}\right)\right)$ with several galaxy properties (independent variables): $\log \Sigma_{\mathrm{d}, 0}, \log h_{\mathrm{d}}\left(\right.$ or $\left.\log M_{\mathrm{bar}}\right)$, $(B-K)$ color, morphological type $T$, and $\log f_{\mathrm{g}}$. We applied backward step-wise multiple linear regressions, where Ftests are used to compute the significance of each independent. The elimination of non-significant variables leads us to a bidimensional linear correlation, where the two more significant independents are $\Sigma_{\mathrm{d}, 0}$ and $T$, although it could also be $\Sigma_{\mathrm{d}, 0}$ and $(B-K)$. The correlation is:

$\left(V_{\mathrm{d}, \mathrm{m}} / V_{\mathrm{t}, \mathrm{m}}\right) \propto \Sigma_{\mathrm{d}, 0} 0.20 \pm 0.02 T^{0.01 \pm 0.003}$, 
with the intercept equal to $10^{-0.72 \pm 0.06}$ and an adjusted correlation coefficient $R=0.79\left(\Sigma_{\mathrm{d}, 0}\right.$ is in unities of $\left.M_{\odot} \mathrm{pc}^{-2}\right)$. The dominant correlation is with $\Sigma_{\mathrm{d}, 0}$. Notice that although a direct linear regression renders $\left(V_{\mathrm{d}, \mathrm{m}} / V_{\mathrm{t}, \mathrm{m}}\right) \propto \Sigma_{\mathrm{d}, 0} 0.14 \pm 0.02$, with an intercept of $10^{-0.54 \pm 0.04}$ and $R=0.75$, as is seen in Eq. (6), the correlation significance is improved slightly introducing $T$, although the slope for $T$ is negligible. The reason for this is that $\Sigma_{\mathrm{d}, 0}$ and $T$ are not completely independent among them; $\Sigma_{\mathrm{d}, 0}$ (anti)correlates slightly with $T$.

The most important result of our analysis is that $\left(V_{\mathrm{d}, \mathrm{m}} / V_{\mathrm{t}, \mathrm{m}}\right)$ depends mainly on $\Sigma_{\mathrm{d}, 0}$. In the past, the conclusions of various observational studies were that the disk and halo mass fractions in the optical parts of disk galaxies depend on the SB and/or luminosity (or scale radius). Applying a direct linear multidimensional regression to $\left(V_{\mathrm{d}, \mathrm{m}} / V_{\mathrm{t}, \mathrm{m}}\right)$ with $\Sigma_{\mathrm{d}, 0}$ and $h_{\mathrm{d}}$ as independents, we find that:

$\left(V_{\mathrm{d}, \mathrm{m}} / V_{\mathrm{t}, \mathrm{m}}\right) \propto \Sigma_{\mathrm{d}, 0}{ }^{0.12 \pm 0.02} h_{\mathrm{d}}^{-0.07 \pm 0.03}$,

with $R=0.75$. The large uncertainty in the slope of $h_{\mathrm{d}}$ shows that the significance of this variable is small, in agreement with the result of the F-test.

We arrive to similar conclusions by making use of the fundamental plane (FP) of disk galaxies. An extensive analysis of the luminous and baryonic FPs for our sample will be presented elsewhere. For the baryonic case and applying the direct linear regression fitting, we obtain that $V_{\mathrm{t}, \mathrm{m}} \propto \Sigma_{\mathrm{d}, 0} 0.37 \pm 0.02 h_{\mathrm{d}} 0.57 \pm 0.03$ with $R=0.95$. Combining this relation with the definition of $\left(V_{\mathrm{d}, \mathrm{m}} / V_{\mathrm{t}, \mathrm{m}}\right) \propto\left[\Sigma_{\mathrm{d}, 0} h_{\mathrm{d}}\right]^{1 / 2} / V_{\mathrm{t}, \mathrm{m}}$, Eq. (7) is recuperated.

We explore now any dependence of the $\left(V_{\mathrm{d}, \mathrm{m}} / V_{\mathrm{t}, \mathrm{m}}\right)$ ratio on more direct observational quantities. We repeat the step-wise multiple regressions using now as independents $\log \Sigma_{K, 0}$ and $\log h_{K}$ instead of $\log \Sigma_{\mathrm{d}, 0}$ and $\log h_{\mathrm{d}}$, besides $(B-K), T$, and $\log f_{\mathrm{g}}$. The statistical analysis shows again that only two independents are significant: $\Sigma_{K, 0}$ and $(B-K)$ or $\Sigma_{K, 0}$ and $T$. The most significant correlation is:

$\left(V_{\mathrm{d}, \mathrm{m}} / V_{\mathrm{t}, \mathrm{m}}\right) \propto \Sigma_{K, 0}{ }^{0.16 \pm 0.02}(B-K)^{-0.08 \pm 0.02}$,

with $R=0.76$. The regressions for $\Sigma_{K, 0}$ and $T$, and $\Sigma_{K, 0}$ and $h_{K}$ give: $\left(V_{\mathrm{d}, \mathrm{m}} / V_{\mathrm{t}, \mathrm{m}}\right) \propto \Sigma_{K, 0}{ }^{0.17 \pm 0.02} T^{0.014 \pm 0.004}(R=0.75)$, and $\left(V_{\mathrm{d}, \mathrm{m}} / V_{\mathrm{t}, \mathrm{m}}\right) \propto \Sigma_{K, 0}{ }^{0.11 \pm 0.01} h_{K}{ }^{-0.09 \pm 0.03}(R=0.72)$, respectively. In the latter case, note the large uncertainty in the slope of $h_{\mathrm{d}}$. The trends seen above (Eq. (7)) with the composite quantities $\Sigma_{\mathrm{d}, 0}$ and $h_{\mathrm{d}}$ remain approximately similar when using the observables $\Sigma_{K, 0}$ and $h_{K}$. We have also repeated the statistical analysis, this time neglecting the gas contribution, that is, $V_{\mathrm{d}, \mathrm{m}}$ was calculated only from $\Sigma_{\mathrm{s}, 0}$ and $h_{\mathrm{s}}\left(=h_{K}\right)$. In this case, the correlation of $\left(V_{\mathrm{d}, \mathrm{m}} / V_{\mathrm{t}, \mathrm{m}}\right)$ with the surface density becomes even slightly steeper than in the case including the disk gas parameters. Therefore, our conclusion about the dependence of $\left(V_{\mathrm{d}, \mathrm{m}} / V_{\mathrm{t}, \mathrm{m}}\right)$ on the disk surface density and its small dependence on the scale radius is robust w.r.t. the the assumptions made to calculate $\Sigma_{\mathrm{d}, 0}$ and $h_{\mathrm{d}}$ from the observational data (see Sect. 2.2): the stellar $\mathrm{M} / \mathrm{L}$ ratio $\gamma_{K}$, and the disk gas parameters, inferred from the total gas mass.

It is important to remark that if the galaxy sample is restricted to a relatively small range of luminosities and/or SBs, then apparent anti-correlations between $\left(V_{\mathrm{d}, \mathrm{m}} / V_{\mathrm{t}, \mathrm{m}}\right)$ and $h_{\mathrm{d}}$, and luminosity or mass appear (in Figs. 7 and 8 we show related effects for the mass-to-luminosity ratio). Therefore, for samples limited in luminosity and/or $\mathrm{SB}$, the $\left(V_{\mathrm{d}, \mathrm{m}} / V_{\mathrm{t}, \mathrm{m}}\right)$ ratio would seem to be larger for smaller galaxies. In fact, even for a complete sample, a slight trend in this direction appears, but it is probably due to the dependence of $\left(V_{\mathrm{d}, \mathrm{m}} / V_{\mathrm{t}, \mathrm{m}}\right)$ on $\Sigma_{\mathrm{d}, 0}$ and the fact that smaller galaxies are typically also of lower SB.

\subsection{Observations and models: Maximum disk?}

Observations and models show that the $\left(V_{\mathrm{d}, \mathrm{m}} / V_{\mathrm{t}, \mathrm{m}}\right)$ ratio changes among galaxies mainly depending on their surface densities (brightnesses): it decreases monotonically as the galaxy is of lower surface density (Fig. 3a). The horizontal lines in Fig. 3a show the range of maximum disk according to Sacket (1997), $\left(V_{\mathrm{d}} / V_{\mathrm{t}}\right)_{2.2 h_{\mathrm{d}}}=0.85 \pm 0.10$, which for the $\left(V_{\mathrm{d}, \mathrm{m}} / V_{\mathrm{t}, \mathrm{m}}\right)$ ratio should be considered only as an approximation for low SBs). Notice that some fraction of the $o b$ served galaxies of ultra-high and high $S B$ are described by maximum or marginally maximum disks, while LSB galaxies tend to be sub-maximum disks. Indeed several detailed observational studies of rotation curve shapes and SB profiles for HSB galaxies show that they tend to be maximum disks (e.g., Corsini et al. 1999; Salucci \& Persic 1999; Palunas \& Williams 2000). Theoretical arguments, including the swing amplifier constraints or bar slowing down due to dynamical friction also suggest a dominion of luminous matter in the center of HSB galaxies (see Bosma 2002 for a review and references therein). On the other hand, for LSB galaxies, a maximum disk solution demands too high stellar M/L ratios, which for reasonable stellar population models imply non-realistic color indexes for the disks (de Blok et al. 1997, 2001). The results in Fig. 3 complement all these previous works, unifying HSB and LSB galaxies in a continuity of decreasing luminous-to-total mass ratio within the optical part of the galaxy as the disk SB decreases. Nevertheless, this ratio varies significantly even for a fixed SB. Below we explore whether other galaxy properties play any significant role in defining the luminous-to-dark matter ratio.

From the theoretical side, for disks formed within $\Lambda \mathrm{CDM}$ halos assuming $f_{\mathrm{d}}=0.05$, only those with the highest surface densities could be described by the maximum disk solution. Galaxy models with moderate $\Sigma_{\mathrm{d}, 0}\left(\sim 400 M_{\odot} \mathrm{pc}^{-2}\right)$ have sub-maximal disks, with $\left(V_{\mathrm{d}, \mathrm{m}} / V_{\mathrm{t}, \mathrm{m}}\right) \approx 0.66 \pm 0.06$ (for the NFW halos, $\left(V_{\mathrm{d}, \mathrm{m}} / V_{\mathrm{t}, \mathrm{m}}\right) \approx\left(V_{\mathrm{d}} / V_{\mathrm{t}}\right)_{2.2 h_{\mathrm{d}}}$ almost ever, as shown in Fig. 2d). If $f_{\mathrm{d}}$ is decreased to the more realistic values of 0.03-0.02 (Avila-Reese et al. 2002), then models result with even lower $\left(V_{\mathrm{d}, \mathrm{m}} / V_{\mathrm{t}, \mathrm{m}}\right)$ ratios. The arrows in panel $\mathrm{b}$ of Fig. 3 indicate how much the $\left(V_{\mathrm{d}, \mathrm{m}} / V_{\mathrm{t}, \mathrm{m}}\right)$ ratio of the models would decrease if instead of $f_{\mathrm{d}}=0.05, f_{\mathrm{d}}=0.03$ is used. In this panel the means per $\Sigma_{\mathrm{d}, 0}$ bins and their corresponding standard deviations are also plotted. The shaded region is for observations, while the region within solid lines is for the models. The thick dotted and solid lines connect the bin means of both groups, respectively.

Observations and models show a large and roughly similar scatter in the $\left(V_{\mathrm{d}, \mathrm{m}} / V_{\mathrm{t}, \mathrm{m}}\right)-\Sigma_{\mathrm{d}, 0}$ plot (Fig. 3). For the former, part of this scatter is due to the combination of observational uncertainties in the measurements of $V_{\mathrm{t}, \mathrm{m}}, \Sigma_{K, 0}, h_{K}$, and 
$M_{\mathrm{HI}}$ propagated in the calculations of $\left(V_{\mathrm{d}, \mathrm{m}} / V_{\mathrm{t}, \mathrm{m}}\right)$ and $\Sigma_{\mathrm{d}, 0}$ (see Graham 2002 for uncertainty estimates for some of the observational data). The residuals of the observational $\left(V_{\mathrm{d}, \mathrm{m}} / V_{\mathrm{t}, \mathrm{m}}\right)$ $\Sigma_{\mathrm{d}, 0}$ relation do not correlate in a significant way with any other galaxy parameter, like $f_{\mathrm{g}}, M_{\mathrm{bar}}, V_{\mathrm{t}, \mathrm{m}}$, morphological type, integral $(B-K)$ color. Nevertheless, we find some trend for redder, earlier type, and more massive galaxies to have on average smaller $\left(V_{\mathrm{d}, \mathrm{m}} / V_{\mathrm{t}, \mathrm{m}}\right)$ ratios. The trends with the type and color would increase slightly if one takes into account that, as the galaxy is of earlier type and redder, the thicker its disk is. The thicker the disk, the lower the $\left(V_{\mathrm{d}, \mathrm{m}} / V_{\mathrm{t}, \mathrm{m}}\right)$ ratio is. Notice that the observations have been uniformly corrected for disk thickness for all the galaxies $\left(5 \%\right.$ in $\left.\left(V_{\mathrm{d}, \mathrm{m}} / V_{\mathrm{t}, \mathrm{m}}\right)\right)$. The trends just mentioned can be explained by our models.

For the models, the scatter in the $\left(V_{\mathrm{d}, \mathrm{m}} / V_{\mathrm{t}, \mathrm{m}}\right)-\Sigma_{\mathrm{d}, 0}$ relation for constant $f_{\mathrm{d}}$ is produced mainly by variations in the halo structure (concentration) and the mass (see Fig. 2b). Since more concentrated halos are the product of earlier mass assembling (Avila-Reese et al. 1998), the disks formed within these halos with a gas accretion rate proportional to the MAH, will be redder. Thus, for a given $\Sigma_{\mathrm{d}, 0}$, redder disks are expected to have lower $\left(V_{\mathrm{d}, \mathrm{m}} / V_{\mathrm{t}, \mathrm{m}}\right)$ ratios. The weak correlation with the mass was already explained in Sect. 3.1. These effects can be interpreted as weak deviations from homology and show a connection between halo structure and luminous evolution. Unfortunately, as mentioned above, the observational uncertainty is too large to allow a direct comparison of models and observations regarding the correlations of the residuals. Nevertheless, at least the trends seem to be in the same direction, showing the good predictive capability of the galaxy evolutionary models of FA2000.

The comparison of observations and models in panels a and $b$ of Fig. 3 shows some agreement among them, but on average models fall below observations. For $f_{\mathrm{d}}=0.03$, the difference increases (see the arrows in Fig. 3b). In panel a we also reproduce the line corresponding to the pseudo-isothermal model with $f_{\mathrm{d}}=0.05\left(M_{\mathrm{v}}=3.5 \times 10^{11} M_{\odot}, r_{\mathrm{c}}=3.9 \mathrm{kpc}\right)$ presented in Fig. 3a, and add results for two other masses: $M_{\mathrm{v}}=3.5 \times 10^{10} M_{\odot}\left(r_{\mathrm{c}}=1.9 \mathrm{kpc}\right.$, upper line $)$ and $M_{\mathrm{v}}=$ $3.5 \times 10^{12} M_{\odot}\left(r_{\mathrm{c}}=7.7 \mathrm{kpc}\right.$, lower line $)$; the core radii are in accordance to inferences by Firmani et al. (2001), see Sect. 3.1. As was noted previously (Figs. 2a and 2b), the major difference between cuspy, gravitationally contracted, NFW and pseudo-isothermal halos in the $\left(V_{\mathrm{d}, \mathrm{m}} / V_{\mathrm{t}, \mathrm{m}}\right)-\Sigma_{\mathrm{d}, 0}$ plane is at high surface densities. The observations lie actually in between both halo models, specially at high surface densities.

The galaxies formed within $\Lambda \mathrm{CDM}$ halos seem to be somewhat less dominated by disk than observations suggest, specially when $f_{\mathrm{d}}=0.03$. The average difference in this case would be roughly of $10 \%$. On the other hand, a pseudoisothermal halo yields a $\left(V_{\mathrm{d}, \mathrm{m}} / V_{\mathrm{t}, \mathrm{m}}\right)$ ratio larger than observational inferences for HSB galaxies (and a steeper increase of the $\left(V_{\mathrm{d}, \mathrm{m}} / V_{\mathrm{t}, \mathrm{m}}\right)$ ratio with $\left.\Sigma_{\mathrm{d}, 0}\right)$. These differences might decrease by using smaller core radii than assumed here or by introducing the disk gravitational drag over the pseudoisothermal halo. The fact that the $\left(V_{\mathrm{d}, \mathrm{m}} / V_{\mathrm{t}, \mathrm{m}}\right)$ ratio continues increasing with $\Sigma_{\mathrm{d}, 0}$ for ultra-HSB observed galaxies could be pointing to that either the disk gravitational drag on the halo is not as efficient as in the models (the gravitational drag is the responsible that $\left(V_{\mathrm{d}, \mathrm{m}} / V_{\mathrm{t}, \mathrm{m}}\right)$ tends to be constant as $\Sigma_{\mathrm{d}, 0}$ increases to high values) or to that some physical process is expanding continuously the halo core (self-interacting CDM for instance).

We conclude that the predictions of the $\Lambda \mathrm{CDM}$ scenario regarding the amounts of dark and luminous matter in disk galaxies are in marginal agreement with observations, specially if $f_{\mathrm{d}}<0.05$. However, the way in which the amounts of dark and luminous matter change as different galaxy properties vary $\left(\Sigma_{\mathrm{d}, 0}\right.$ in particular) is in good agreement with observations. It seems that minor modifications to the inner structure of the $\Lambda \mathrm{CDM}$ halos is enough to conciliate models with observations. In FA00 we have shown that the introduction of soft cores in agreement with inferences from the rotation curves of LSB galaxies, produce a $\sim 10 \%$ increase of the $\left(V_{\mathrm{d}, \mathrm{m}} / V_{\mathrm{t}, \mathrm{m}}\right)$ ratio (on average) for HSB galaxies. These models would be in better agreement with observations in Fig. 3.

\section{Dynamical-to-baryonic mass and mass-to-light ratios}

In the previous section we have investigated the $\left(V_{\mathrm{d}, \mathrm{m}} / V_{\mathrm{t}, \mathrm{m}}\right)$ ratio from observed and model galaxies and determined how it depends on different galaxy properties. As shown in Fig. 2, panels $\mathrm{c}$ and $\mathrm{d},\left(V_{\mathrm{d}, \mathrm{m}} / V_{\mathrm{t}, \mathrm{m}}\right)$ seems to be a good tracer of the amounts of luminous and dark matter in disk galaxies. In this section we will study directly the dynamical-to-baryonic and dynamical-to-stellar mass ratios, $M_{\mathrm{dyn}} / M_{\mathrm{bar}}$ and $M_{\mathrm{dyn}} / M_{\mathrm{s}}$, as well as the mass-to-light ratios. The latter is the ratio most directly obtained from observations and it has been determined previously in the literature, mainly in the $B$-band (e.g., Salucci et al. 1991; Karachentsev 1991; Roberts \& Haynes 1994; Graham 2002). We remark that $L_{K}$ and $L_{B}$ refer to the total galaxy luminosities, i.e. disk+bulge. Therefore, $M_{\mathrm{S}}$ and $M_{\mathrm{bar}}$ are the total disk+bulge stellar and baryonic masses, respectively. In general, while the $\left(V_{\mathrm{d}, \mathrm{m}} / V_{\mathrm{t}, \mathrm{m}}\right)$ ratio refers to the disk contribution ( $V_{\mathrm{d}, \mathrm{m}}$ has been calculated from the disk SB profile (Eq. (5)), the $M_{\text {dyn }} / M_{\text {bar }}$ ratio refers to the total galaxy contribution (disk + bulge). Thus, from the observational point of view, the information contained in $M_{\text {dyn }} / M_{\text {bar }}$ is slightly different to that contained in $\left(V_{\mathrm{d}, \mathrm{m}} / V_{\mathrm{t}, \mathrm{m}}\right)$.

In Sect. 3, Eq. (4), we have shown the theoretical relation between our observational quantity $\left(V_{\mathrm{d}, \mathrm{m}} / V_{\mathrm{t}, \mathrm{m}}\right)$ and $M_{\mathrm{dyn}} / M_{\mathrm{bar}}$ defined at $r_{\text {peak }}$, the radius where the rotation curve peaks. For disk-dominated galaxies, the radii where the disk and total velocities peak are approximately the same, at $2.2 h_{\mathrm{d}}(x=2.2)$, and the disk mass fraction at this radius is $f_{\mathrm{L}}=0.64$. For halodominated galaxies, the departure of $x$ and $f_{\mathrm{L}}$ from 2.2 and 0.64 will depend mainly on the halo mass distribution; in any case, both will increase, in such a way that the ratio $x / f_{\mathrm{L}}$ will only slightly change (increase). For example, for NFW halos, $x / f_{\mathrm{L}}$ remains almost constant $(\approx 2.2 / 0.64)$ for almost any case (see Fig. 2d). Thus, we may estimate approximately the $M_{\text {dyn }} / M_{\text {bar }}$ ratio at $2.2 h_{\mathrm{d}}$ by multyplying $M_{\mathrm{dyn}} / M_{\mathrm{bar}} \equiv\left(V_{\mathrm{t}, \mathrm{m}}{ }^{2} h_{\mathrm{d}} / G\right) / M_{\mathrm{bar}}$ by $2.2 / 0.64=3.44$. We may also estimate $M_{\mathrm{dyn}} / M_{\mathrm{bar}}$ at $5 h_{\mathrm{d}}=5 r_{\text {peak }} / x$ (roughly the optical radius of disks). For this, we have to assume that the rotation curve remains flat from its maximum up to this radius, and $M_{\mathrm{dyn}}$ should be multiplied by 5 ; for an exponential disk, the mass at $5 h_{\mathrm{d}}$ is close to the 
total disk mass (99\%). In fact, the outer slope of the rotation curves changes, mainly depending on the disk surface density and luminosity. While some LSB and low-luminosity galaxies may have still increasing rotation curves at $\sim 5 h_{\mathrm{d}}$, HSB and luminous galaxies may present decreasing shapes at this radius (e.g., Casertano \& van Gorkom 1991; Persic et al. 1996; Verheijen 1997). Galaxies with increasing rotation curves at the last measured point were excluded from our sample. The highest SB and luminosity galaxies may have on average a rotation velocity at $5 h_{\mathrm{d}}$ smaller than $V_{\mathrm{t}, \mathrm{m}}$ by a $10-20 \%$. Therefore, the $M_{\text {dyn }} / M_{\text {bar }}$ ratio at $5 h_{\mathrm{d}}$ presented below could be overestimated by this factor for the highest SB and luminosity galaxies.

\subsection{Results}

In panels a-c of Fig. 4 we plot $\left(V_{\mathrm{t}, \mathrm{m}}{ }^{2} h_{\mathrm{d}} / G\right) / M_{\mathrm{bar}}$ vs. $\Sigma_{\mathrm{d}, 0}$, $\left(V_{\mathrm{t}, \mathrm{m}}{ }^{2} h_{\mathrm{d}} / G\right) / M_{\mathrm{s}}$ vs. $\Sigma_{\mathrm{s}, 0}$, and $\left(V_{\mathrm{t}, \mathrm{m}}{ }^{2} h_{\mathrm{d}} / G\right) / L_{K}$ vs. $\Sigma_{K, 0}$, respectively. The axis to the right of each panel was shifted up by a factor of 5 to resemble approximately $M_{\mathrm{dyn}} / M_{\mathrm{bar}}, M_{\mathrm{dyn}} / M_{\mathrm{s}}$, and $M_{\mathrm{dyn}} / L_{K}$ at $5 h_{\mathrm{d}}$, respectively. To obtain these ratios at $2.2 h_{\mathrm{d}}$, the shift should be approximately by a factor of 3.44 . Similar to $\left(V_{\mathrm{d}, \mathrm{m}} / V_{\mathrm{t}, \mathrm{m}}\right)$, the $M_{\mathrm{dyn}} / M_{\mathrm{bar}}$ ratio presents a dependence on $\Sigma_{\mathrm{d}, 0}$, with a poor correlation with $h_{\mathrm{d}}$. Applying a direct multidimensional linear regression to $M_{\mathrm{dyn}} / M_{\mathrm{bar}}$ as a function of $\Sigma_{\mathrm{d}, 0}$ and $h_{\mathrm{d}}$ (Log-Log), we arrive to the same conclusion of the previous section: the dynamical-to-baryonic mass ratio in disk galaxies depends mainly on $\Sigma_{\mathrm{d}, 0}$ but weakly on scale $\left(h_{\mathrm{d}}\right.$ or $\left.M_{\mathrm{bar}}\right)$. The correlation obtained from the observations is

$M_{\text {dyn }} / M_{\text {bar }} \propto \Sigma_{\text {d, } 0}{ }^{-0.31 \pm 0.03} h_{\mathrm{d}}^{-0.05 \pm 0.05}$,

with $R=0.8$. This is consistent with Eq. (7) if $M_{\text {dyn }} / M_{\text {bar }} \propto$ $\left(V_{\mathrm{d}, \mathrm{m}} / V_{\mathrm{t}, \mathrm{m}}\right)^{-2}$.

The direct linear regression of $\log \left(M_{\mathrm{dyn}} / M_{\mathrm{bar}}\right)$ with

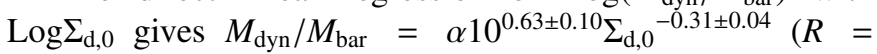
0.74), with $\alpha=5$ (solid line in Fig. 4) and $\alpha=3.44$ for this ratio defined at 5 and $2.2 h_{\mathrm{d}}$, respectively. The ratio of baryon to dark matter, $M_{\mathrm{bar}} / M_{\mathrm{dm}}$, is related to $M_{\mathrm{dyn}} / M_{\mathrm{bar}}$ by: $M_{\mathrm{bar}} / M_{\mathrm{dm}}=1 /\left(M_{\mathrm{dyn}} / M_{\mathrm{bar}}-1\right)$. Thus, on average the $\left(M_{\mathrm{bar}} / M_{\mathrm{dm}}\right)_{2.2 h_{\mathrm{d}}}$ ratio for ultra-HSB $\left(\Sigma_{\mathrm{d}, 0} \approx 2000 M_{\odot} \mathrm{pc}^{-2}\right)$, moderate-HSB $\left(\Sigma_{\mathrm{d}, 0} \approx 400 M_{\odot} \mathrm{pc}^{-2}\right)$, and very-LSB $\left(\Sigma_{\mathrm{d}, 0} \approx\right.$ $50 M_{\odot} \mathrm{pc}^{-2}$ ) galaxies are 2.5, 0.8 and 0.25 , respectively. For the MW and M31 galaxies, $\left(M_{\mathrm{bar}} / M_{\mathrm{dm}}\right)_{2.2}=0.74$ and 1.34, respectively.

The anti-correlation of $M_{\text {dyn }} / M_{\text {bar }}$ with the surface density becomes steeper (and tighter) when one passes from the baryonic to the stellar disk or the $K$-band (panels b and c). A direct multidimensional lineal regression shows that $M_{\mathrm{dyn}} / M_{\mathrm{S}} \propto \Sigma_{\mathrm{s}, 0}{ }^{-0.51 \pm 0.04} h_{K}{ }^{-0.13 \pm 0.06}$ and $M_{\mathrm{dyn}} / L_{K} \propto$ $\Sigma_{K, 0}{ }^{-0.56 \pm 0.03} h_{K}{ }^{-0.16 \pm 0.06}$ ( $R=0.86$ and 0.89 , respectively). This change is because galaxies with lower surface densities have typically higher gas fractions than the higher surface density ones. A contrary effect happens when one passes from stellar masses and densities to the $B$-band luminosities and SBs: lower surface density galaxies are typically more luminous in the $B$-band (bluer) than the higher surface density galaxies with the same masses. Therefore, the $M_{\mathrm{dyn}} / L_{B}$ vs. $\Sigma_{B, 0}$ relation (Fig. 5a) becomes shallower and noisier than the $M_{\text {dyn }} / M_{\mathrm{s}}$ vs. $\Sigma_{\mathrm{s}, 0}$ relation. Nevertheless, we find that $M_{\mathrm{dyn}} / L_{B}$

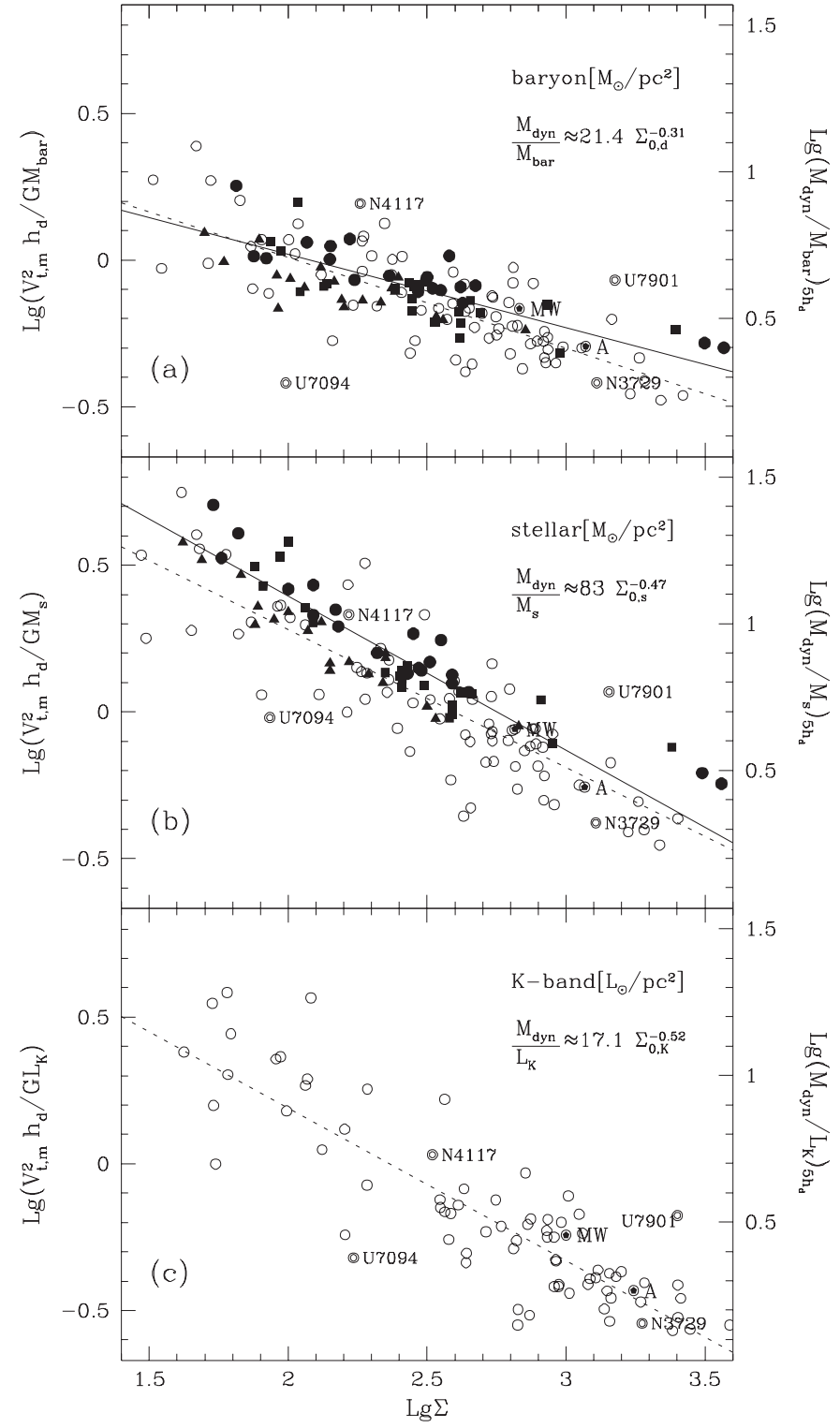

Fig. 4. a) Dynamical-to-baryonic mass ratio vs. the baryonic surface density for observations (empty circles) and models (same symbols as in Fig. 3). The left axis is the quantity inferred directly from the observational data, while the right axis is this quantity multiplied by 5 ; in the assumption that the rotation curve remains constant up to $5 h_{\mathrm{d}}$, this corresponds to $M_{\mathrm{dyn}} / M_{\text {bar }}$ at $5 h_{\mathrm{d}}$. Solid and dotted lines are the linear regressions for models and observations, respectively. For observations, the fitting (at $5 h_{\mathrm{d}}$ ) is showed in the upper right. b) Same as in panel a) but for the stellar mass and surface density. c) Same as in panel a) but for the $K$-band luminosity and SB. Only observations are showed in this panel.

still (anti)correlates significantly with $\Sigma_{B, 0}$, and does not correlate with $h_{B}$ (Fig. 6a). A multidimensional linear regression shows that

$M_{\mathrm{dyn}} / L_{B} \propto \Sigma_{B, 0}{ }^{-0.37 \pm 0.04} h_{B}^{0.03 \pm 0.06}$

with $R=0.77$.

It should be noticed that if the sample is strongly restricted in magnitudes, a spurious correlation of $M_{\mathrm{dyn}} / L_{B}$ with $h_{B}$ appears. For instance, if the data in Fig. $6 a\left(M_{\text {dyn }} / L_{B}\right.$ vs. $\left.h_{B}\right)$ are sorted into three groups according to their $B$-band magnitudes, 
then each group show an apparent correlation. We also have calculated the residuals of the $M_{\mathrm{dyn}} / L_{B}-\Sigma_{B, 0}$ and $h_{B}-\Sigma_{B, 0}$ relations. There is not any dependence among them. Contrary to this, and using the FP, Graham (2002) arrived to the conclusion that $M_{\text {dyn }} / L_{B}$ does not depend on $\Sigma_{B, 0}$ but with $h_{B}$. In Sect. 4.2 we discuss the reasons of this apparent difference.

The correlations we have found for $M_{\mathrm{dyn}} / L_{K}$ and $\Sigma_{K, 0}$, and for $M_{\mathrm{dyn}} / L_{B}$ and $\Sigma_{B, 0}$ imply the existence of a dependence of $\mathrm{L}_{B} / \mathrm{L}_{K}$ on both $\Sigma_{B, 0} / \Sigma_{K, 0}$ and $\Sigma_{K, 0}$. With some scatter, this dependence can be written as

$(B-K) \approx 0.36(B-K)_{0}-0.19 \mu_{K, 0}$,

which agrees with a direct comparison of these observable parameters. The disk center is typically redder than the average disk color and the difference increases for redder galaxies (e.g., de Jong 1996c; Verheijen 1997).

In Fig. 7a we explore whether the $M_{\text {dyn }} / M_{\text {bar }}$ ratio (defined at $\left.5 h_{\mathrm{d}}\right)$ correlates with the integral $(B-K)$ color. Apparently there is no significant direct correlation, but a weak anticorrelation (see also Graham 2002). This is mostly because redder galaxies are commonly of higher SB. As was seen above, galaxies with higher SB are less dark matter dominated than galaxies with lower SB. However, for a given SB, one expects that redder galaxies (assembled earlier) are more dark matter dominated, at a given SB, than the bluer galaxies (Avila-Reese \& Firmani 2000). This is because dark halos assembled by an early collapse are more concentrated than halos assembled by extended accretion. In Fig. 7b, results from the same evolutionary models presented in Sect. 3 are shown in terms of the $(B-V)$ color. The data were sorted into three groups according to their disk surface densities. For the lower surface density models, $M_{\text {dyn }} / M_{\text {bar }}$ correlates with the color as expected. For higher surface densities the correlation tends to disappear because of the effect of the surface density and the "saturation" of the dark-to-baryonic mass ratio at high SBs. This is due to the disk gravitational drag over the halos (otherwise, the bluer HSB models could have lower $M_{\text {dyn }} / M_{\text {bar }}$ ratios). In panel a the observational data has also been sorted into the same three groups according to their disk surface densities as in panel $b$. The trend for the low surface density galaxies is similar than that for the models. At high surface densities observations also agree roughly with models. However, several of the highest surface density and reddest galaxies appear with lower $M_{\text {dyn }} / M_{\text {bar }}$ ratios than models. These galaxies, having the most dominant disks and (probably) the more concentrated halos, reveal that dark matter is not as dominant as in the case of disks formed within NFW halos.

In Fig. 4, panels a and b, the data from the evolutionary models are also included (solid symbols; but notice that there are no model predictions in the $K$ band). Solid and dashed lines are direct linear regressions to the observational and model data, respectively. As in the case of the $\left(V_{\mathrm{d}, \mathrm{m}} / V_{\mathrm{t}, \mathrm{m}}\right)$ ratio, models agree marginally with observations, yielding on average higher $M_{\mathrm{dyn}} / M_{\mathrm{bar}}$ and $M_{\mathrm{dyn}} / M_{\mathrm{s}}$ ratios than observations, specially for the highest surface density galaxies. For example, for the groups of ultra-HSB and moderate-HSB galaxies mentioned above, the model $\left(M_{\mathrm{bar}} / M_{\mathrm{dm}}\right)_{2.2 h_{\mathrm{d}}}$ ratios are on average 1.4 and 0.6 (with a large scatter), which means approximately

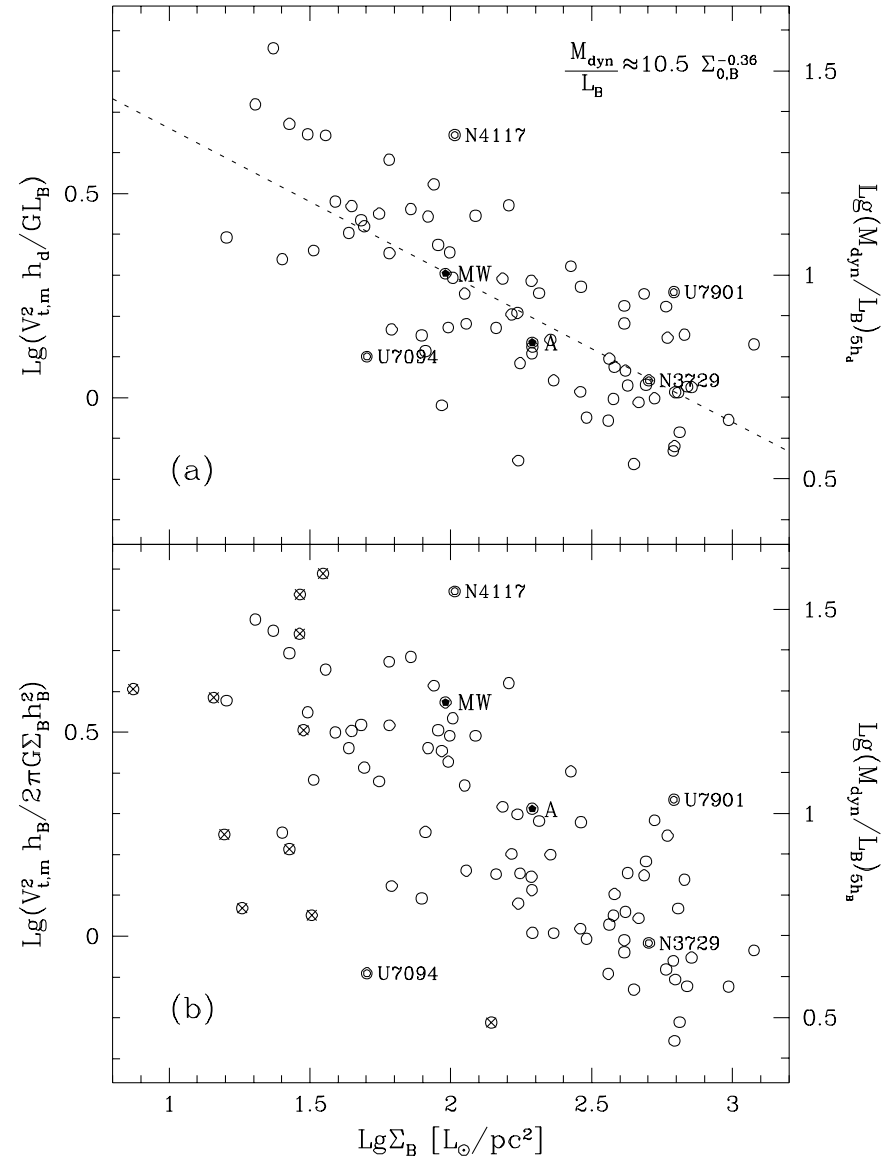

Fig. 5. a) Same as Fig. 4, but for $B$-band luminosity and SB, and only for observational data. b) Same as panel a) but using the definitions of Graham (2002; see text) and including some LSB galaxies with large bulges also considered by Graham (circles with crosses). Notice how the scatter in the correlation seen in panel a) increases in panel b).

1.8 and 1.25 times more dark matter dominated than observations, respectively. For the LSB galaxies, the ratios are roughly similar. The differences between observations and models increase if $f_{\mathrm{d}}$ is fixed to values smaller than 0.05 . The halos of observed galaxies have on average less dark matter content in the center than the $\Lambda \mathrm{CDM}$ model suggests. It should be also noted that the differences between models and observations decrease when passing from stellar to baryonic (stars+gas) parameters. This is because models have slightly higher gas fractions than observations (FA00). In our models all the gas is cold, while the observations suggest that some fraction of the gas may be ionized in the outer disks. Therefore, this gas in not detected in the HI observations.

\subsection{Comparison with previous works}

Using the tilt of the optimal fundamental plane (FP) of disk galaxies, Graham (2002) concluded that $M_{\mathrm{dyn}} / L_{B}$ depends on $h_{B}$ rather than on $\Sigma_{B, 0}$. Since we share almost the same observational sources, our results broadly agree with those of Graham (2002) if we follow his FP analysis. In this indirect way to estimate the dependence of $M_{\mathrm{dyn}} / L_{B}$ on $\Sigma_{B, 0}$ and $h_{B}$, the multidimensional regression is applied not to $M_{\mathrm{dyn}} / L_{B}$, but to $V_{\mathrm{t}, \mathrm{m}}$ (the FP). This is one of the reasons behind the different conclusions achieved in Graham's paper. From his data, one 


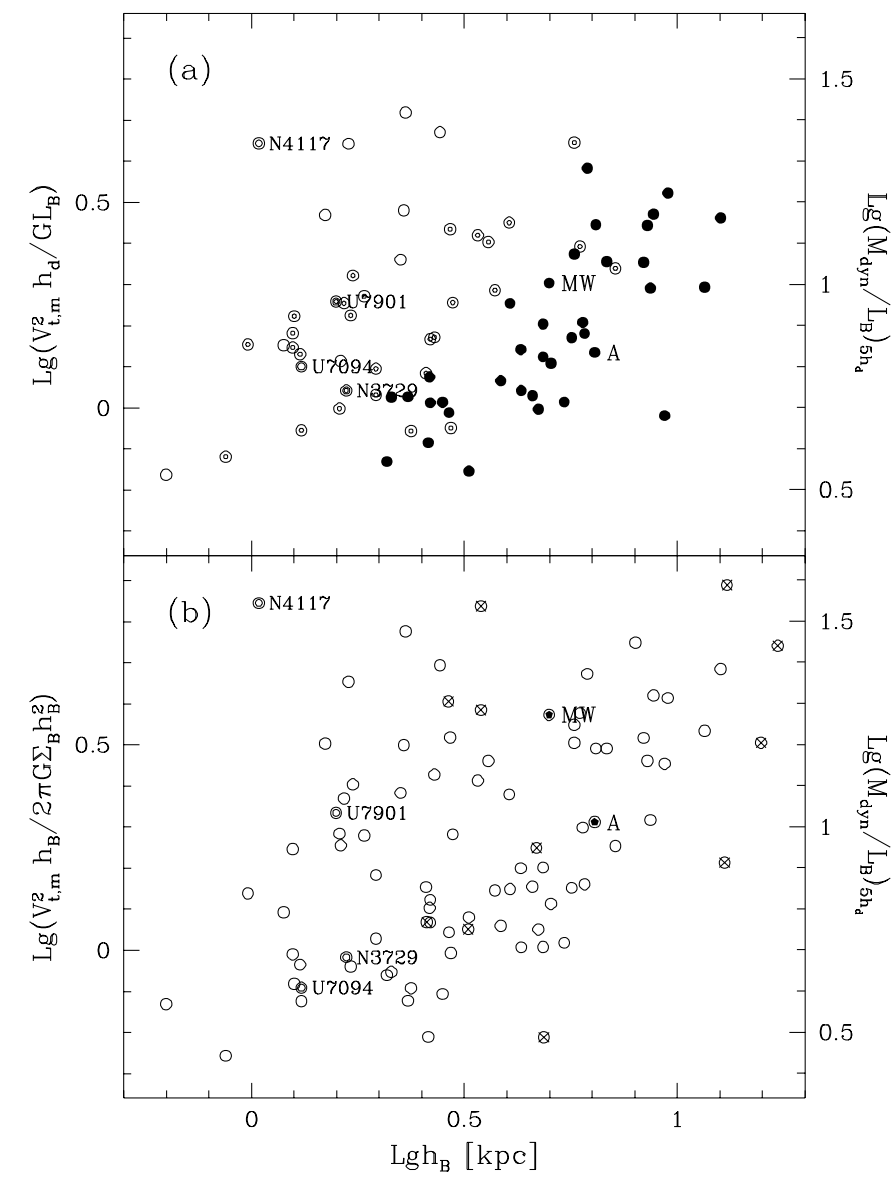

Fig. 6. a) Same as in Fig. 5a but for $h_{B}$ instead of $\Sigma_{B, 0}$ in the $x$-axis. The data are sorted into three groups according to their $B$-band magnitudes. The corresponding ranges are: $4.0 \times 10^{8}-2.5 \times 10^{9} L_{B}$ (open symbols), $2.5 \times 10^{9}-1.6 \times 10^{10} L_{B \odot}$ (targeted symbols), and $1.6 \times 10^{10}-1.0 \times 10^{11} L_{B_{\odot}}$ (solid symbols). Although for all the data is not significant, for the data sorted in luminosity ranges, a correlation between the mass-to-light ratio and $h_{B}$ appears. b) Same as in Fig. $5 \mathrm{~b}$ but for $h_{B}$ instead of $\Sigma_{B, 0}$ in the $x$-axis. A correlation appears for all the data in this panel (calculated according to Graham 2002).

sees that if the mass-to-light ratio dependence on $\Sigma_{B, 0}$ and $h_{B}$ is determined directly ("kinematic" $M / L$ ratio) instead of through the FP (the "photometric" $M / L$ ratio), then the dependences on $\Sigma_{B, 0}$ and $h_{B}$ increases and decreases slightly, respectively (see his Fig. 4), and the result approaches to what we have reported in the previous subsection.

Other reasons to explain the difference in results are the way to calculate $M_{\text {dyn }}$ and $L_{B}$. Graham (2002) uses the disk scale radius in the current band while we define a unique physical $M_{\mathrm{dyn}}$, using the baryonic disk scale radius $h_{\mathrm{d}}$. Rather than the measured $L_{B}$, Graham uses $\Sigma_{B, 0} h_{B}{ }^{2}$. Figures 5 and 6 , panel $b$, show our data using the same definitions as in Graham (2002). We also include in these plots LSB galaxies with large bulges from the sample of Beijersbergen (1999) and de Blok et al. (1998) (circles with crosses; for these galaxies there is available information only in the $B$-band), which were used by Graham. It is clearly seen that the dependence of $M_{\text {dyn }} / L_{B}$ on $\Sigma_{B, 0}$ becomes weaker in panel b of Fig. 5 than in panel a. On the other hand, as in Graham (2002), some correlation with $h_{B}$ appears now (compare panels a and b of Fig. 6). Thus, our results agree broadly with those of Graham (2002) when we apply to the data his technique and definitions, and when we include the peculiar sub-sample of LSB galaxies with large bulges.

Nevertheless, we think that our analysis and the conclusion in Sect. 4.1 that $M_{\text {dyn }} / L_{B}$ anti-correlates with $\Sigma_{B, 0}$ and does not depend on $h_{B}$ or $L_{B}$ is physically correct. If we use the result of Graham (2002), it is easy to show that $(B-R) \approx-0.25(B-$ $R)_{0}-\left(0.09 \mu_{B}+0.12 M_{B}\right)$, which implies an anti-correlation of the central color with the integral color of galaxies, contrary to that inferred from direct observational comparisons.

For a sample of 114 spirals, Karachentsev (1991) has found that the ratio of dynamical mass-to- $L_{B}$ does not depend on $L_{B}$ but does anti-correlate with the $B$-band SB, in agreement with our result (see also Giovanardi \& Hunt 1988). The anticorrelation in the data of Karachentsev $\left(M_{\text {dyn }} / L_{B} \propto \Sigma_{B, 0}^{-s}\right.$, $s \approx 0.5)$ is steeper than we have found. We expect that the slope $s$ would decrease if the velocity-dependent internal extinction correction applied here is applied to those data.

Salucci et al. (1991) inferred the ratio $M_{\text {dyn }} / M_{\text {bar }}$, by using $B$-band data for a sample of HSB galaxies and assuming constant central SB. They concluded that this ratio decreases roughly as $L_{B}^{-0.4}$. However, as we have mentioned in Sect. 3.3, a spurious correlation among $\left(V_{\mathrm{d}, \mathrm{m}} / V_{\mathrm{t}, \mathrm{m}}\right)$ and luminosity (mass) appears if the sample is restricted in SB. The same applies for the $M_{\text {dyn }} / M_{\text {bar }}$ ratio. Salucci et al. (1991) assumed constant SB and their sample was indeed limited to HSB galaxies only. Furthermore, we expect that the correlation with $L_{B}$ in Salucci et al. will diminish if a luminosity-dependent internal extinction coefficient is applied to the data.

Finally, from the analysis of a sample of optical rotation curves from HSB late-type spirals, Persic \& Salucci (1988, 1990) have found that $M_{\mathrm{dyn}} / M_{\mathrm{bar}}$ at $3.2 h_{\mathrm{d}}$ decreases with $L_{B}$ as $L_{B}^{-0.4}$. The method is conceptually different from that used here and it is based on a different observational information. Our results (observational and theoretical) show that $\left(M_{\mathrm{dyn}} / M_{\mathrm{bar}}\right)_{2.2 h_{\mathrm{d}}}$ does not correlate with $M_{\text {bar }}$ or $L_{B}$. The main assumption we have made to infer the $M_{\mathrm{dyn}} / M_{\mathrm{bar}}$ ratio at $2.2 h_{\mathrm{d}}$ from observations is that $x / f_{\mathrm{L}}(x)$ in Eq. (4) remains almost constant with the SB or luminosity. This is indeed true for disks formed inside CDM halos (or for any halo with a circular velocity that does not increases significantly after the maximum of the disk component $)$, where $\left(V_{\mathrm{d}, \mathrm{m}} / V_{\mathrm{t}, \mathrm{m}}\right) \approx\left(V_{\mathrm{d}} / V_{\mathrm{t}}\right)_{2.2 h_{\mathrm{d}}}$ as seen in Fig. $2 \mathrm{~d}$. Since our analysis in Sect. 3 of the trends of $\left(V_{\mathrm{d}, \mathrm{m}} / V_{\mathrm{t}, \mathrm{m}}\right)$ on several galaxy parameters has shown a reasonable agreement between observations and models, we may speculate that the galaxy halos do not depart significantly from the NFW mass distribution, in particular at intermediate radii. Nevertheless, more tests are needed in order to see whether $x / f_{\mathrm{L}}(x)$ indeed does not depend on SB/luminosity.

A more direct comparison of the results from our observational sample and the inferences of Persic and collaborators can be done through the $\left(V_{\mathrm{d}, \mathrm{m}} / V_{\mathrm{t}, \mathrm{m}}\right)$ ratio, a quantity that was calculated from the available data without introducing any assumption about $x / f_{\mathrm{L}}(x)$. The analysis presented in Sect. 3.3 shows that $\left(V_{\mathrm{d}, \mathrm{m}} / V_{\mathrm{t}, \mathrm{m}}\right)$ for our sample does not depend significantly on mass or luminosity, but on SB. On the opposite, the mass decomposition of the universal rotation curve implies a strong correlation of $\left(V_{\mathrm{d}, \mathrm{m}} / V_{\mathrm{t}, \mathrm{m}}\right)$ with luminosity (see Fig. 6 in 

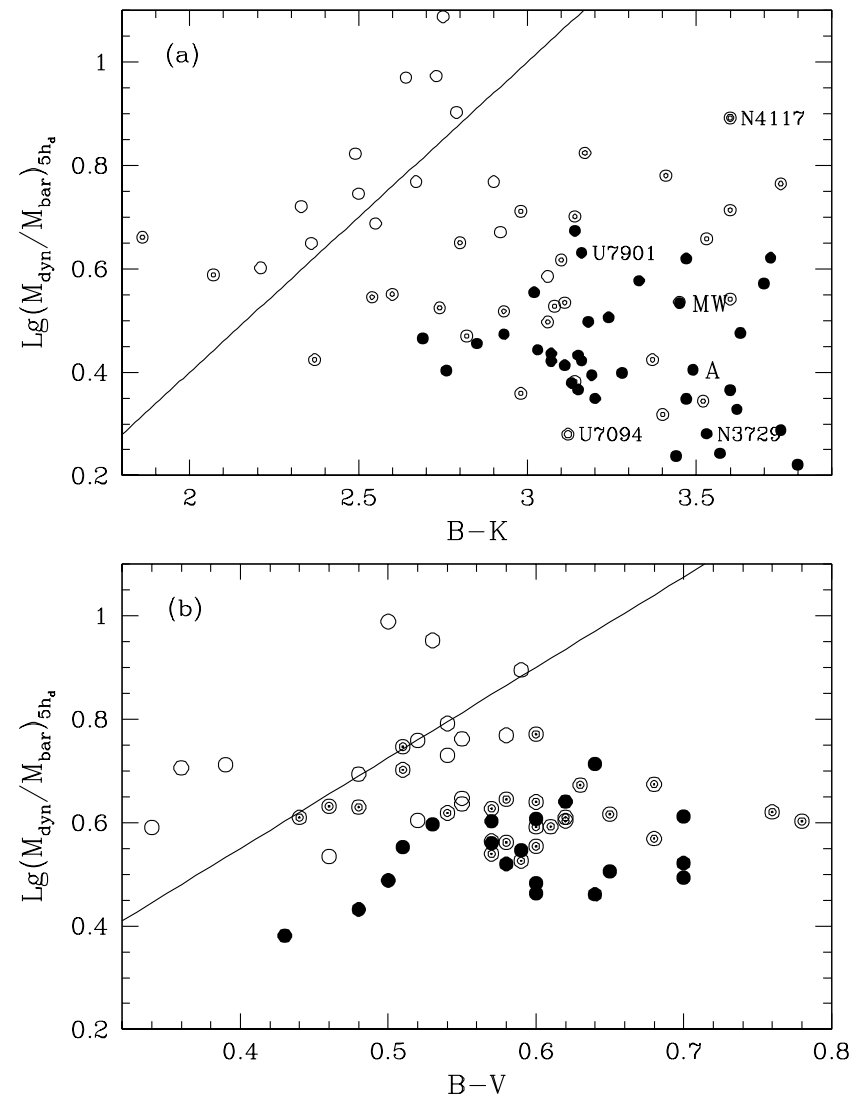

Fig. 7. a) Approximate dynamical-to-baryonic mass ratio at $5 h_{\mathrm{d}}$ vs. integral $(B-K)$ color for observational data. No correlation, if any, an anti-correlation, is seen. However, for limited ranges of surface density, a correlation appears as this density is lower. The data in the plot were sorted into three groups according to their disk central surface densities: open, targeted and solid symbols are for low, intermedium and high surface densities, respectively (we use surface densities instead of $L_{K}$ luminosity in order to have the same ranges for the model data presented in panel b). The solid line is an eye-fit to the low surface density group. b) Same as in panel a) but for the model data and for $(B-V)$ instead of $(B-K)$. See text for explanations of this figure.

Persic et al. 1996), while the correlation with SB was not explored in that work.

The investigation in detail of the differences on the results obtained with the two different methods is out of the scope of the present paper. Our results suggest that the SB should be taken into account in the kinematical method as an important parameter for the shape of the rotation curves. On the other hand, we notice that, according to our sample data, the $h_{B} / h_{\mathrm{d}}$ (or $h_{B} / h_{K}$ ) ratio increases on average with $L_{B}$. The results of Persic $\&$ Salucci $(1988,1990)$ and Persic et al. (1996) are based on the determination of the slope of the rotation curve at 3.2 scale radii. They used the optical (mostly $B$-band) disk scale radii instead of $h_{K}$ or $h_{\mathrm{d}}$; the latter are more directly related to the gravitational field of the disk than $h_{B}$. Since the slope of the rotation curve is sensible to the radius at which it is measured, a systematic shift of this radius toward larger values as the luminosity increases, will introduce an (extra) anti-correlation of this slope with the luminosity, if the the rotation curves are actually decreasing as the galaxy is more luminous or of higher $\mathrm{SB}$. We speculate that, by using the more physical $h_{K}$ or $h_{\mathrm{d}}$ radii rather than $h_{B}$ or $h_{I}$, the anti-correlation of the rotation curve slope at 3.2 scale radii with $L_{B}$ found by the mentioned authors will become weaker.

In general, most of the previous works on the dark and luminous contents in disk galaxies did not take into account that these contents vary significantly among galaxies, depending on the disk SB. We have shown that the ratio of totalto-luminous matter content within the optical regions of the galaxies decreases as the SB increases, without any significant (anti)correlation with galaxy scale.

\section{Summarizing discussion}

We have presented a compilation of normal disk galaxies with available photometric parameters in both the $B$ and $K$ bands, rotation curves and/or HI-velocity line-widths, and HI integral fluxes. A total of 78 galaxies have been selected as useful for our analysis. We made the data (luminosities, disk central SBs and scale radii, maximum rotation velocity, disk gaseous mass) uniform and used them to further calculate several stellar and baryonic galaxy parameters. With this sample we explored the ratios of dark to luminous mass (and luminosity) within disk galaxies and how these ratios vary with the galaxy properties. The inferences obtained here are alternative to other more direct methods, such as the rotation curve decomposition and the statistical analysis of rotation curve shapes. A detailed comparison of our results with those of more direct methods is not within the scope of the present paper, but deserves a future analysis. In this paper, our observational results were compared with model predictions and analyzed further in the light of the behavior of the models. We next discuss the main conclusions of this work.

- The $\left(V_{\mathrm{d}, \mathrm{m}} / V_{\mathrm{t}, \mathrm{m}}\right)$ ratio inferred from observations depends mainly on the disk central surface density, $\Sigma_{\mathrm{d}, 0}\left(\right.$ or $\left.\Sigma_{K, 0}\right)$, increasing approximately as $\Sigma_{\mathrm{d}, 0} 0.15$. Only a fraction of HSB and ultra-HSB galaxies in our sample fall in the range of maximum $\operatorname{disk}\left(\left(V_{\mathrm{d}, \mathrm{m}} / V_{\mathrm{t}, \mathrm{m}}\right) \approx 0.85 \pm 0.10\right)$; as the disk central surface density decreases, the galaxies become more dark matter dominated, with the lowest SB galaxies having $\left(V_{\mathrm{d}, \mathrm{m}} / V_{\mathrm{t}, \mathrm{m}}\right)$ ratios around 0.5 . The MW and $\mathrm{M} 31$ galaxies have $\left(V_{\mathrm{d}, \mathrm{m}} / V_{\mathrm{t}, \mathrm{m}}\right)=0.67$ and 0.84 , respectively. In previous works, by using the analysis of rotation curve shapes, a "democratic" solution to the discussion about maximum or sub-maximum disk was found, in the sense that there are galaxies with rotation curves highly dominated by the disk component and other ones dominated by the halo component (e.g., Persic et al. 1996). The main driving parameter in these works was the luminosity or mass. Our results show instead that the driving parameter is the disk surface brightness or density.

- Although the $\left(V_{\mathrm{d}, \mathrm{m}} / V_{\mathrm{t}, \mathrm{m}}\right)$ ratio depends mainly on $\Sigma_{\mathrm{d}, 0}$ $\left(\Sigma_{K, 0}\right)$, a large scatter is observed in the $\left(V_{\mathrm{d}, \mathrm{m}} / V_{\mathrm{t}, \mathrm{m}}\right)-\Sigma_{\mathrm{d}, 0}$ plot. Interestingly, we find some trend of decreasing $\left(V_{\mathrm{d}, \mathrm{m}} / V_{\mathrm{t}, \mathrm{m}}\right)$ as the galaxy is redder, of earlier type and more luminous (massive). These results hint us about a connexion between the luminous and morphological galaxy properties to the dark halo properties. The latter ones are related to the initial (cosmological) conditions.

- To gain a deeper understanding of the observations, we have compared them with models of disk galaxy formation and 
evolution in the $\Lambda$ CDM cosmogony (FA00). For a disk-to-total halo mass ratio $f_{\mathrm{d}}=0.05$, the models agree marginally with the observational data in the $\left(V_{\mathrm{d}, \mathrm{m}} / V_{\mathrm{t}, \mathrm{m}}\right)$ vs. $\Sigma_{\mathrm{d}, 0}$ plane (Fig. 3$)$. Models have on average slightly lower $\left(V_{\mathrm{d}, \mathrm{m}} / V_{\mathrm{t}, \mathrm{m}}\right)$ ratios than observations, the difference becoming larger for the highest values of $\Sigma_{\mathrm{d}, 0}$. For $f_{\mathrm{d}}=0.03$, the differences increase slightly. These differences suggest that real galaxies may have less dark matter in the center than disks formed within $\Lambda \mathrm{CDM}$ halos. The observations in the $\left(V_{\mathrm{d}, \mathrm{m}} / V_{\mathrm{t}, \mathrm{m}}\right)-\Sigma_{\mathrm{d}, 0}$ plot lie indeed in between models with cuspy NFW halos and pseudo-isothermal halos with shallow cores, specially at the highest surface densities, where the differences between both halo models become large. Nevertheless, the differences between observations and the $\Lambda \mathrm{CDM}$ models are modest, and the dependences of $\left(V_{\mathrm{d}, \mathrm{m}} / V_{\mathrm{t}, \mathrm{m}}\right)$ on different galaxy properties (in particular $\left.\Sigma_{\mathrm{d}, 0}\right)$ for observations and these models agree rather well (e.g., see thick dotted and solid lines in Fig. 3b). Therefore, only a minor modification to the inner structure of the $\Lambda \mathrm{CDM}$ halos seems to be necessary to remedy the differences with the observations.

A possible mechanism to form soft cores in the CDM halos, without altering their MAHs and outer structures, is the gravothermal expansion produced in the core if the CDM particles are allowed to self-interact. Cosmological $N$-body simulations for self-interacting CDM particles with a modest cross section inversely proportional to the particle relative velocity, have shown to be an attractive alternative to produce soft cores in agreement with observational inferences from dwarfs to galaxy cluster scales, keeping at the same time the successful predictions of the "standard" $\Lambda$ CDM paradigm (Colín et al. 2002). The constant expansion of the core could explain why the $\left(V_{\mathrm{d}, \mathrm{m}} / V_{\mathrm{t}, \mathrm{m}}\right)$ ratios of ultra-HSB galaxies is so high in spite of the strong gravitational drag of the disk on the halo (see Fig. 2). Models of disk galaxy evolution within the self-interacting $\Lambda \mathrm{CDM}$ halos will show whether self-interaction leads to a better agreement with observations than in the case of collisionless CDM particles.

- The models show that, eventhough more massive (luminous) systems have typically less concentrated dark halos than smaller ones, for a given $\Sigma_{\mathrm{d}, 0}$, the former are more dark matter dominated (smaller $\left(V_{\mathrm{d}, \mathrm{m}} / V_{\mathrm{t}, \mathrm{m}}\right)$ ratios) than the latter. This trend, also seen in observations, is easily explained in the light of the models (Sects. 3.1 and 3.2.2). On the other hand, for a given mass, the $\left(V_{\mathrm{d}, \mathrm{m}} / V_{\mathrm{t}, \mathrm{m}}\right)$ ratio of disks in more concentrated halos is lower than in the less concentrated ones. More concentrated halos are typically the result of earlier mass assembling, therefore the disks formed within them are redder because the gas infall rate at latter epochs is low. Thus, the trend of redder galaxies presenting smaller $\left(V_{\mathrm{d}, \mathrm{m}} / V_{\mathrm{t}, \mathrm{m}}\right)$ ratios, at least for LSB galaxies, is understandable in the models and it might explain the same trend found for the observations. In general, the scatter of the observational data in the $\left(V_{\mathrm{d}, \mathrm{m}} / V_{\mathrm{t}, \mathrm{m}}\right)-\Sigma_{\mathrm{d}, 0}$ plane is only slightly larger than for the models (Fig. 3b). Most of this scatter is explained in the light of the models, and the rest is perhaps due to the observational uncertainties.

- We also have presented the dynamical-to-baryon mass ratio of models and observations (Fig. 4). To estimate this ratio at 2.2 and $5 \times h_{\mathrm{d}}$ from the kind of observations we have used here, one has to assume that $V_{\mathrm{t}, \mathrm{m}}$ occurs at $2.2 h_{\mathrm{d}}$ for all the galaxies, or that $V_{\mathrm{t}, \mathrm{m}}$ remains constant up to $5 h_{\mathrm{d}}$, respectively. The $M_{\text {dyn }} / M_{\text {bar }}$ ratio of observations and models depends mainly on $\Sigma_{\mathrm{d}, 0}$ and does not depend on scale $\left(h_{\mathrm{d}}\right.$ or $\left.M_{\mathrm{bar}}\right)$. These trends sharpen when using $\left(M_{\mathrm{s}}, \Sigma_{\mathrm{s}, 0}\right)$ and $\left(L_{K}, \Sigma_{K, 0}\right)$ instead of $\left(M_{\mathrm{bar}}, \Sigma_{\mathrm{d}, 0}\right)$. But when passing to $\left(L_{B}, \Sigma_{B, 0}\right)$, the relation between $M_{\mathrm{dyn}} / L_{B}$ and $\Sigma_{B, 0}$ becomes shallower and noisier than in the other cases $\left(M_{\mathrm{dyn}} / M_{\mathrm{s}}\right.$ vs. $\Sigma_{\mathrm{s}, 0}$, for example). Nevertheless, we find that $M_{\text {dyn }} / L_{B}$ still (anti)correlates significantly with $\Sigma_{B, 0}$, and does not correlate with $h_{B}$ (or $L_{B}$ ). The $M_{\mathrm{dyn}}$-to- $M_{\mathrm{bar}}$, $-M_{\mathrm{S}},-L_{K}$, and $-L_{B}$ ratios at $5 h_{\mathrm{d}}$ for the lowest SB galaxies are approximately $8,14,10.5$ and 18 , respectively, while for the highest SB galaxies are 2, 2, 1.5, and 5, respectively (Figs. 5 and 6). The $M_{\mathrm{dyn}} / M_{\mathrm{bar}}$ ratio for the highest SB model galaxies (maximum difference with observations) is around 2.4. This means that for the highest SB galaxies, models predict halos $\sim 1.7$ more massive at $5 h_{\mathrm{d}}$ than inferred from observations (at $2.2 h_{\mathrm{d}}$ this difference is of $\sim 1.8$ ).

- The $M_{\text {dyn }} / M_{\text {bar }}$ ratio does not correlate with $L_{B}$ (or $M_{\text {bar }}$ ) as was found previously in works based on the statistical analysis of rotation curve shapes, where the determination of the rotation curve slope at some typical radius $\left(3.2 h_{B}\right.$ in these works) is crucial. We have noted that the correlation of $h_{B} / h_{\mathrm{d}}\left(\right.$ or $\left.h_{B} / h_{K}\right)$ on $L_{B}$ could contribute significantly to an anti-correlation of the slope with $L_{B}$, in the understanding that $h_{\mathrm{d}}$ (or $h_{K}$ ) is the physical radius to be used. We also note that the strong dependence of the $\left(V_{\mathrm{d}, \mathrm{m}} / V_{\mathrm{t}, \mathrm{m}}\right)$ ratio on luminosity inferred from the universal rotation curve is not reproduced by our data analysis. The point is that two different methods, using different samples, arrive at different conclusions: we find that the fractions of dark and luminous matter within the disks depend mainly on the SB or surface density, while in works based on the analysis of the rotation curve shapes, the main parameter is the luminosity or mass. In fact luminosity and SB are correlated, but the determination of which of them is the dominant is an important issue to understand galaxy formation and evolution. If the luminosity (or mass) is the main parameter, then disk formation in $\Lambda \mathrm{CDM}$ models could be not realistic or intermediate astrophysical processes like a strongly mass-dependent feedback could be relevant. It is important that future works solve the controversy stated here.

- The correlations of the mass-to-light ratios with SB in the $B$ and $K$ bands found here imply that the integral color $(B-K)$ correlates with the central disk color $(B-K)_{0}$ (Eq. (11)), in accord to a direct comparison of both parameters. We have also found that as galaxies are of less $\mathrm{SB}$, a more significant correlation among the $M_{\text {dyn }} / M_{\text {bar }}$ ratio and $(B-K)$ appears (Fig. 7). According to the models, galaxies are redder in part because they formed in more concentrated dark halos (halos that collapsed earlier on average). Therefore, for a given SB, $M_{\text {dyn }} / M_{\text {bar }}$ tends to be larger (more domination of dark matter) for redder galaxies. However, for disks with high SBs, the $M_{\text {dyn }} / M_{\text {bar }}$ ratio tends to be more constant than for low SBs (due to the disk gravitational drag over the halo), in such a way that the correlation with the color minimizes. The similar features found between models and observations (compare panels $a$ and $b$ of Fig. 7) suggests that this is a plausible explanation for the observational trend. 
- The good agreement found between models and observations in the comparisons presented in the present paper favors the $\Lambda \mathrm{CDM}$ scenario, under the assumptions that disk galaxies are formed by gas accretion rather than mergers, and that during the collapse of the gas, detailed angular momentum conservation is obeyed. In the light of the results presented here, the main difficulty of this scenario is an apparent excess of dark matter within the disk optical radius. A minor modification to the physics of the formation of the $\Lambda \mathrm{CDM}$ halos seems to be enough to overcome this difficulty.

Acknowledgements. We have made use of the Lyon-Meudon Extragalactic Database (LEDA), and the NASA/IPAC Extragalactic Database (NED) which is operated by the Jet Propulsion Laboratory, California Institute of Technology, under contract with NASA. We thank the referee for useful comments and for suggesting several improvements to the manuscript. This work was funded by CONACyT grant 33776-E to V.A.

\section{References}

Avila-Reese, V., \& Firmani, C. 2000, RevMexAA, 36, 23

Avila-Reese, V., Firmani, C., \& Hernández, X. 1998, ApJ, 505, 37

Avila-Reese, V., Firmani C., \& Zavala, J. 2002, ASP Conf. Ser., 282, 137

Avila-Reese, V, Firmani, C., Klypin, A., \& Kravtsov, A. 1999, MNRAS, 309, 507

Beijersbergen, M., de Blok, W. J. G., \& van der Hulst, J. M. 1999, A\&A, 351, 903

Bell, E., Barnaby, D., Bower, R. G., et al. 2000, MNRAS, 312, 470

Bell, E., \& de Jong, R. S. 2001, ApJ, 550, 212

Bosma, A. 1978, Ph.D. Thesis, Groningen Univ.

Bosma, A. 1981a, AJ, 86, 1791

Bosma, A. 1981b, AJ, 86, 1825

Bosma, A. 2002, ASP Conf. Ser., 275, 23

Broeils, A. H., \& van Woerden, H. 1994, A\&AS, 107, 129

Bruzual, A. G. 1983, ApJ, 273, 105

Bullock, J. S., Dekel, A., Kolatt, T. S., et al. 2001, ApJ, 555, 240

Burlak, A. N., Gubina, V. A., \& Tyurina, N. V. 1997, Astro. Lett., 23, 522

Casertano, S., \& van Gorkom, J. H. 1991, AJ, 101, 1231

Colín, P., Avila-Reese, V., Valenzuela, O., \& Firmani, C. 2002, ApJ, 581,777

Corsini, E. M., Pizzella, A., Sarzi, M., et al. 1999, A\&A, 342, 671

Cox, A. N. 2000, Allen's Astrophysical Quantities, ed. A. N. Cox (Springer \& AIP Press), 4th edition

Dalcanton, J. J., Spergel, D. N., \& Summers, F. J. 1997, ApJ, 482, 659

de Blok, W. J. G., van der Hulst, J. M., \& Bothun, G.D. 1995, MNRAS, 274, 235

de Blok, W. J. G., McGaugh, S. S., \& van der Hulst, J. M. 1996, MNRAS, 283, 18

de Blok, W. J. G., \& McGaugh, S. S. 1997, MNRAS, 290, 533

de Blok, W. J. G., McGaugh, S. S., \& Rubin, V. C. 2001, AJ, 122, 2396

de Jong, R. S. 1996a, A\&A, 313, 45

de Jong, R. S. 1996b, A\&A, 313, 377

de Jong, R. S. 1996c, A\&AS, 118, 557

de Jong, R. S., \& van der Kruit, P. C. 1994, A\&AS, 106, 451

Eke, V. R., Navarro, J. F., \& Frenk, C. S. 1998, ApJ, 503, 569

Eke, V. R., Navarro, J. F., \& Steinmetz, M. 2001, ApJ, 554, 114

Firmani, C., \& Avila-Reese, V. 2000, MNRAS, 315, 457 (FA00)

Firmani, C., Avila-Reese, V., \& Hérnandez, X. 1997, ASP Conf. Ser., 117,424
Firmani, C., D’Onghia, E., Chincarini, G., Hérnandez, X., \& AvilaReese, V. 2001, MNRAS, 321, 713

Flores, R. A., Primack J. R., Blumenthal G. R., \& Faber S. M. 1993, ApJ, 412, 443

Freeman, K. C. 1970, ApJ, 160, 811

Ghigna, S., Moore, B., Governato, et al. 1998, MNRAS, 300, 146

Gilmore, G., King, I. R., \& van der Kruit, P. C. 1990, The Milky Way as a galaxy, ed. University Science Books.

Giovanardi, C., \& Hunt, L. K. 1988, AJ, 95, 408

Giovanelli, R., Haynes, M. P., Salzer, J. J., et al. 1994, AJ, 107, 2036

Graham, A. W. 2002, MNRAS, 334, 721

Hernández Toledo, H. M., Dultzin-Hacyan, D., Gonzalez, J., \& Sulentic, J. W. 1999, AJ, 118, 108

Hiromoto, N., Maihara, T., Oda, N., \& Okuda, H. 1983, PASJ, 35, 413

Jansen, R. A., Franx, M., Fabricant, D., \& Caldwell, N. 2000, ApJSS, 126, 271

Karachentsev, I. D. 1991, SvAL, 17, 367

Kent, S. M. 1986, AJ, 91, 1301

Kent, S. M., Dame, T. M., \& Fazio, G. 1991, ApJ, 378, 131

Marsh, K. A., \& Helou, G. 1995, ApJ, 445, 599

McGaugh, S. S., \& de Blok, W. J. G. 1997, ApJ, 481, 689

Mo, H. J., Mao, S., \& White, S. D. M. 1998, MNRAS, 295, 319

Navarro, J. F., Frenk, C. S., \& White, S. D. M. 1997, ApJ, 490, 493

Olivier, S. S., Blumenthal, G. R., \& Primack, J. R. 1991, MNRAS, 252, 102

Palunas, P., \& Williams, T. B. 2000, AJ, 120, 2884

Paturel, G., Andernach, H., Bottinelli, L., et al. 1997, A\&AS, 124, 109

Persic, M., \& Salucci, P. 1988, MNRAS, 234, 131

Persic, M., \& Salucci, P. 1990a, MNRAS, 245, 577

Persic, M., \& Salucci, P. 1990b, MNRAS, 247, 349

Persic, M., \& Salucci, P. 1991, MNRAS, 248, 325

Persic, M., Salucci, P., \& Stel, F. 1996, MNRAS, 281, 27

Poggianti, B. M. 1997, A\&AS, 122, 399

Roberts, M. S., \& Haynes, M. P. 1994, ARA\&A, 32, 115

Rogstad, D. H., \& Shostak, G. S. 1972, ApJ, 176, 315

Rubin, V. C., Thonnard, N., \& Ford, W. K. 1980, ApJ, 238, 471

Rubin, V. C., Thonnard, N., Ford, W. K., \& Burstein, D. 1982, ApJ, 261, 439

Rubin, V. C., Burstein, D., Ford, W. K., \& Thonnard, N. 1985, ApJ, 289, 81

Sackett, P. D. 1997, ApJ, 483, 103

Salucci, P., \& Borriello, A. 2001, in Proc. of the International Conf. DARK 2000 (Heidelberg, Germany), 12

Salucci, P., \& Persic, M. 1999, A\&A, 351, 442

Salucci, P., Ashman, K. M., \& Persic, M. 1991, ApJ, 379, 89

Schlegel, D. J., Finkbeiner, D. P., \& Davis, M. 1998, ApJ, 500, 525

Shustov, B., Wiebe, D., \& Tutukov, A. 1997, A\&A, 317, 397

Tinsley, B. M. 1981, MNRAS, 194, 63

Tully, R. B., \& Verheijen, M. A. W. 1997, ApJ, 484, 145

Tully, R. B., Pierce, M. J., Huang, J., et al. 1998, AJ, 115, 2264

van Albada, T. S., \& Sancisi, R. 1986, Philos. Trans. Ser. A, 320, 447

van den Bosch, F. C. 1998, ApJ, 507, 601

van den Bosch, F. C. 2000, ApJ, 530, 177

van den Bosch, F. C. 2002, MNRAS, 332, 456

Verheijen, M. A. W. 1997, Ph.D. Thesis, Groningen University

Verheijen, M.A.W., \& Sancisi, R. 2001, A\&A, 370, 765

Wang, B., \& Heckman, T. M. 1996, ApJ, 457, 645

Wainscoat, R. J., \& Cowie, L. L. 1992, AJ, 103, 332

Wechsler, R. H., Bullock, J. S., Primack, J. R., Kravtsov, A. V., \& Dekel, A. 2002, ApJ, 568, 52

Young, J. S., \& Knezek, P. M. 1989, ApJ, 347, L55 


\section{Online Material}




\section{Appendix A}

In this Appendix we describe the construction of the disk+halo composite models. The overall procedure closely resembles that presented by Mo et al. (1998).

The disk is assumed to be in centrifugal equilibrium, to be thin, and to have an exponential surface density distribution, $\Sigma_{\mathrm{d}, 0}(r)=\Sigma_{\mathrm{d}, 0} \exp \left(-r / h_{\mathrm{d}}\right)$, where the central surface density $\Sigma_{\mathrm{d}, 0}$ and scale radius $h_{\mathrm{d}}$ are related to the total disk mass by $M_{\text {bar }}=2 \pi \Sigma_{\mathrm{d}, 0} h_{\mathrm{d}}{ }^{2}$. The circular velocity is calculated according to Eq. (5). The angular momentum of the disk, neglecting its gravitational effect, is:

$J_{d}=2 \pi \int V_{h}(r) \Sigma_{\mathrm{d}, 0}(r) r^{2} \mathrm{~d} r$

where $V_{\mathrm{h}}(r)$ is the halo circular velocity. It is assumed that the disk forms from the baryons trapped within the virialized halo (with a mass fraction $f_{\mathrm{d}}$ ) and that they follow originally the same mass and angular momentum distribution of the halo. If detailed angular momentum conservation is obeyed, then $J_{\mathrm{d}}=$ $J$, where $J$ is the halo angular momentum, and it is related to the dimensionless spin parameter $\lambda$ by:

$\lambda=J|E|^{1 / 2} G^{-1} M_{\mathrm{v}}{ }^{-5 / 2}$,

where $E$ is the total energy of the halo. Now, supplanting these relations into Eq. (12), the disk scale radius $h_{\mathrm{d}}$ is calculated; it will be proportional to $\lambda$. Therefore, given $M_{\mathrm{v}}, f_{\mathrm{d}}, \lambda$ and the halo density profile, the two parameters of the exponential disk in centrifugal equilibrium remain defined. Following, two halo models are analyzed:

Pseudo-isothermal (P-I) sphere. The density and circular velocity profiles of a P-I sphere with core radius $r_{c}$ and constant central density $\rho_{0}$ are given by:

$$
\begin{aligned}
\rho(r) & =\frac{\rho_{0}}{1+\left(r / r_{\mathrm{c}}\right)^{2}}, \\
V_{h}^{2}(r) & =\frac{G M_{h}(r)}{r}=\left[4 \pi G \rho_{0} r_{\mathrm{c}}^{2}\left(1-\frac{r_{\mathrm{c}}}{r} \arctan \left(\frac{r}{r_{\mathrm{c}}}\right)\right)\right]
\end{aligned}
$$

The asymptotic velocity is $V_{\text {asym }}=\left(4 \pi G \rho_{0} r_{\mathrm{c}}^{2}\right)^{0.5}$. According to the cosmological spherical collapse model, at the epoch of virialization of the last shell of an overdense homogeneous sphere, its radius (virial radius, $r_{\mathrm{v}}$ ) is such that $\left\langle\rho\left(<r_{\mathrm{v}}\right)\right\rangle=\Delta_{\mathrm{c}} \rho_{\mathrm{u}}$, where $\left\langle\rho(<r)>\right.$ is the average density of the sphere at $r, \Delta_{\mathrm{c}}$ is a constant, and $\rho_{\mathrm{u}}$ is the matter density of the universe at that epoch. For the present epoch and for the $\Lambda \mathrm{CDM}$ cosmology used here, $\Delta_{\mathrm{c}}=334$ (Eke et al. 1998). The mass contained within $r_{\mathrm{v}}$ is the virial (halo) mass, $M_{\mathrm{v}}$. Taking into account that $r_{\mathrm{v}} / r_{\mathrm{c}} \gg 1$, it is easy to find that $V_{\text {asym }} \approx\left(G M_{\mathrm{v}} / r_{\mathrm{v}}\right)^{1 / 2} \approx$ $0.014\left(M_{\mathrm{V}} / M_{\odot}\right)^{1 / 3} \mathrm{~km} \mathrm{~s}^{-1}$, and that $E \approx-\left(M_{\mathrm{v}} V_{\text {asym }}^{2}\right) / 2$. At radii larger than $r_{\mathrm{c}}, V_{\mathrm{h}}$ rapidly tends to $V_{\text {asym }}=$ const., and the integral in Eq. (12) can be approximated by $J_{\mathrm{d}} \approx 2 M_{\text {bar }} h_{\mathrm{d}} V_{\text {asym. }}$. Now, from $J_{\mathrm{d}}=J$ and using Eq. (13), $h_{\mathrm{d}}$ can be calculated, $h_{\mathrm{d}} \propto \lambda M_{\mathrm{v}}^{1 / 3} f_{\mathrm{d}}^{-1}$, and $\Sigma_{\mathrm{d}, 0} \propto M_{\mathrm{v}}^{1 / 3} f_{\mathrm{d}}^{3} \lambda^{-2}$.

Once $M_{\mathrm{v}}, f_{\mathrm{d}}$, and $\lambda$ are defined, we calculate the disk parameters $h_{\mathrm{d}}$ and $\Sigma_{\mathrm{d}, 0}$ and use them in Eq. (5) to get the disk velocity component. The halo velocity component is calculated according to Eq. (14). The core radius $r_{c}$ is taken from empirical inferences found by Firmani et al. (2001) from dwarf and
LSB galaxies: $r_{\mathrm{c}}^{\prime} \approx 5.5\left(V_{\text {asym }} / 100 \mathrm{~km} \mathrm{~s}^{-1}\right)^{0.95}$, where we have to take into account that the core radius of the non-singular isothermal model used by them, $r_{\mathrm{c}}^{\prime}$, is 1.45 times the core radius of the pseudo-isothermal model used here, $r_{\mathrm{c}}=r_{\mathrm{c}}^{\prime} / 1.45$.

NFW cosmological halo model. The typical density and circular velocity profiles of the CDM halos are given by:

$$
\begin{aligned}
& \rho(r)=\frac{\rho_{\mathrm{s}}}{\left(r / r_{\mathrm{s}}\right)\left(1+r / r_{\mathrm{s}}\right)^{2}}, \\
& V_{\mathrm{h}}^{2}(r)=\frac{G M_{\mathrm{h}}(r)}{r}=\frac{4 \pi G \rho_{\mathrm{s}} r_{\mathrm{s}}^{3}\left[\ln \left(1+\frac{r}{r_{\mathrm{s}}}\right)-\frac{1}{1+r_{\mathrm{s}} / r}\right]}{r},
\end{aligned}
$$

where the parameters $\rho_{\mathrm{s}}$ and $r_{\mathrm{s}}$ are related. Defining again as above the virial radius $r_{\mathrm{v}}$ and introducing the concentration parameter $c=r_{\mathrm{v}} / r_{\mathrm{s}}$, one obtains that $\rho_{\mathrm{s}}=\left(\Delta_{\mathrm{c}} \rho_{\mathrm{u}} / 3\right) c^{3} g(c)$, where $g(c)=1 /(\ln (1+c)-c /(1+c))$. Now, the halo circular velocity can be expressed in terms of the velocity at the virial radius $\left(V_{\mathrm{v}}=\left(G M_{\mathrm{v}} / r_{\mathrm{v}}\right)^{1 / 2}\right):\left(V_{\mathrm{h}} / V_{\mathrm{v}}\right)^{2}=(1 / w)(\ln (1+c w)-c w /(1+$ $c w)) g(c)$, where $w=r / r_{\mathrm{v}}$. As shown above, from the spherical collapse model one obtains that $V_{\mathrm{v}} \propto M_{\mathrm{v}}{ }^{1 / 3}$. Therefore, given $M_{\mathrm{v}}$ and c, the NFW velocity profile remains defined. In order to calculate the disk parameters, the total halo energy is necessary: $E=-G M_{\mathrm{v}}^{2} f(c) /\left(2 r_{\mathrm{v}}\right)$, where $f(c)$ is a function of the concentration parameter (see Mo et al. 1998) and $r_{\mathrm{v}} \propto V_{\mathrm{v}}$. Cosmological numerical simulations show that c slightly decreases with $M_{\mathrm{v}}$ and has a significant scatter $\left(\Delta \log c_{\mathrm{NFW}} \approx\right.$ 0.18, Bullock et al. 2001).

Gravitational contraction of the NFW halo. The formation of a dense disk inside the cosmological dark halo induces a gravitational contraction of the halo in the inner regions. Under the assumption of radial adiabatic invariance, one may calculate the mean radius $r$ where ends a particle with an initial mean radius $r_{\mathrm{i}}: G M_{\mathrm{f}}(r) r=G M\left(r_{\mathrm{i}}\right) r_{\mathrm{i}}$, where $M_{\mathrm{f}}(r)=M_{\mathrm{d}}(r)+M\left(r_{i}\right)\left(1-f_{\mathrm{d}}\right)$ is the final total mass distribution and $M\left(r_{i}\right)$ is the initial halo (NFW) mass distribution. To calculate the disk mass distribution $M_{\mathrm{d}}(r), h_{\mathrm{d}}$ should be found as described above. But to calculate $h_{\mathrm{d}}$ the total circular velocity $V_{\mathrm{t}}^{2}(r)=V_{\mathrm{d}}^{2}(r)+V_{\mathrm{h}}^{2}(r)$ should be given, where $V_{\mathrm{h}}^{2}(r)=G\left[M_{\mathrm{f}}(r)-M_{\mathrm{d}}(r)\right] / r$. Given $M_{\mathrm{v}}, f_{\mathrm{d}}, \lambda$ and c, an iterative procedure yields $h_{\mathrm{d}}$ and $V_{\mathrm{t}}(r)$ after the halo contraction. This procedure is explained in Mo et. al (1998) and we refer the reader to that paper for more details.

\section{Appendix B}

Here we present the main ingredients of the self-consistent evolutionary models to be studied in this paper. The disk is built up within a growing $\Lambda \mathrm{CDM}$ halo. A special extended PressSchechter approach is used to generate the statistical mass aggregation histories (MAHs) of the halos from the primordial density fluctuation field, and a generalized secondary infall model is applied to calculate the virialization of the accreting mass shells. The evolution and structure of the $\Lambda \mathrm{CDM}$ halos calculated this way agree well with results from cosmological N-body simulations (Avila-Reese et al. 1999); halos assembled through early active MAHs end more concentrated on average than halos with extended MAHs. The mass shells are assumed to have aligned rotation axis with specific angular momentum 
given by $j_{\mathrm{sh}}\left(t_{\mathrm{v}}\right)=\mathrm{d} J\left(t_{\mathrm{v}}\right) / \mathrm{d} M_{\mathrm{v}}\left(t_{\mathrm{v}}\right)$, where $J=\lambda G M_{\mathrm{v}}^{5 / 2} /|E|^{1 / 2}$, $J, M_{\mathrm{v}}$ and $E$ are the total angular momentum, mass and energy of the halo at the shell virialization time $t_{\mathrm{v}}$. The spin parameter, $\lambda$, is assumed to be constant in time. As the result of the assembling of these mass shells, a present day halo ends with an angular momentum distribution close to the (universal) distribution measured by Bullock et al. (2001) in $N$-body simulations. A fraction $f_{\mathrm{d}}$ of the mass of each shell is assumed to cool down and form a disk layer in a dynamical time. The radial mass distribution of the layer is calculated by equating its specific angular momentum to that of its final circular orbit in centrifugal equilibrium (detailed angular momentum conservation is assumed). The superposition of these layers form the disk. The gravitational interaction of disk and halo is calculated using the adiabatic invariance formalism. The local SF is triggered by the Toomre gas gravitational instability criterion and self-regulated by a vertical disk balance between the energy input due to $\mathrm{SNe}$ and the turbulent energy dissipation in the ISM. The SF efficiency depends on the gas surface density determined mainly by $\lambda$, and on the gas accretion rate determined by the cosmological MAH. Finally, we calculate the formation of a secular bulge by using the Toomre instability criterion for the stellar disk. 
J. Zavala et al.: Luminous and dark matter in disk galaxies, Online Material $p 4$

Table 1. Raw and corrected photometric and dynamical parameters of the disk galaxy sample

\begin{tabular}{|c|c|c|c|c|c|c|c|c|c|}
\hline $\begin{array}{l}\text { Name } \\
\mathrm{T}\end{array}$ & $\begin{array}{l}\mathrm{D}^{\mathrm{a}} \\
i\left(^{o}\right)\end{array}$ & $\begin{array}{c}m_{B}^{\mathrm{b}} \\
\log L_{B, c}^{\mathrm{g}}\end{array}$ & $\begin{array}{c}m_{K}^{\mathrm{b}} \\
\operatorname{Lg} L_{K, c}^{\mathrm{g}}\end{array}$ & $\begin{array}{c}\mu_{0, B}^{\mathrm{c}} \\
\operatorname{Lg} \Sigma_{0, B c}^{\mathrm{h}}\end{array}$ & $\begin{array}{c}\mu_{0, K}^{\mathrm{c}} \\
\operatorname{Lg} \Sigma_{0, K c}^{\mathrm{h}}\end{array}$ & $\begin{array}{l}(B-K)^{\mathrm{d}} \\
(B-K)_{0}^{\mathrm{i}}\end{array}$ & $\begin{array}{l}h_{B}^{\mathrm{e}} \\
h_{K}^{\mathrm{e}}\end{array}$ & $\begin{array}{c}\mathrm{W}_{20, c}^{\mathrm{f}} \\
\operatorname{LgM}_{H I}^{\mathrm{j}}\end{array}$ & Ref. $^{k}$ \\
\hline UGC 89 & 67.6 & 12.63 & 8.85 & 22.07 & 17.46 & 3.40 & 9.34 & 516.4 & "1,6" \\
\hline 1 & 49.3 & 10.99 & 11.52 & 1.97 & 2.83 & 4.22 & 5.70 & 9.96 & \\
\hline UGC 242 & 64.6 & 13.92 & 10.30 & 21.26 & 17.28 & 3.24 & 4.29 & 407.3 & "1,6" \\
\hline 6 & 40.6 & 10.43 & 10.90 & 2.35 & 2.96 & 3.59 & 3.48 & 9.75 & \\
\hline UGC 334 & 68.7 & 15.24 & 12.07 & 23.36 & 20.32 & 2.92 & 7.16 & 187.1 & "1,6" \\
\hline 9 & 41.6 & 9.90 & 10.24 & 1.40 & 1.74 & 2.91 & 6.02 & 9.80 & \\
\hline UGC 438 & 66.9 & 12.95 & 9.10 & 20.45 & 16.23 & 3.57 & 4.57 & 547.4 & $" 1,6 "$ \\
\hline 5 & 39.7 & 10.81 & 11.41 & 2.63 & 3.38 & 3.96 & 3.73 & 9.91 & \\
\hline UGC 490 & 66.9 & 13.48 & 9.46 & 21.47 & 17.16 & 3.62 & 6.00 & 471.7 & "1,6" \\
\hline 5 & 46.0 & 10.65 & 11.27 & 2.24 & 2.97 & 3.91 & 4.83 & 10.18 & \\
\hline UGC 628 & 79.8 & 15.58 & 12.52 & 22.86 & 20.39 & 2.73 & 5.73 & 260.7 & "1,6" \\
\hline 9 & 52.4 & 9.93 & 10.20 & 1.49 & 1.63 & 2.40 & 7.20 & 9.82 & \\
\hline UGC 1305 & 39.2 & 12.75 & 8.65 & 22.02 & 17.61 & 3.70 & 6.44 & 513.9 & "1,6" \\
\hline 4 & 36.8 & 10.47 & 11.12 & 2.09 & 2.86 & 4.01 & 5.03 & 9.61 & \\
\hline UGC 1577 & 77.9 & 13.79 & 9.90 & 22.44 & 18.26 & 3.60 & 8.34 & 423.4 & "1,6" \\
\hline 3 & 38.7 & 10.61 & 11.22 & 1.78 & 2.58 & 4.06 & 6.64 & 10.11 & \\
\hline UGC 1719 & 120.3 & 14.08 & 10.09 & 22.45 & 17.73 & 3.53 & 12.65 & 690.4 & "1,6" \\
\hline 3 & 44.2 & 10.95 & 11.54 & 1.86 & 2.75 & 4.29 & 8.34 & 10.13 & \\
\hline UGC 2064 & 62.2 & 14.20 & 10.18 & 22.28 & 18.01 & 3.37 & 6.06 & 328.1 & $" 1,6 "$ \\
\hline 4 & 46.8 & 10.41 & 10.92 & 2.06 & 2.64 & 3.53 & 5.31 & 9.82 & \\
\hline UGC 2081 & 36.8 & 14.50 & 11.55 & 22.31 & 19.44 & 2.67 & 3.40 & 216.5 & "1,6" \\
\hline 6 & 54.7 & 9.66 & 9.90 & 1.69 & 1.99 & 2.82 & 3.46 & 9.33 & \\
\hline UGC 4308 & 52.1 & 13.17 & 9.83 & 21.34 & 17.66 & 3.06 & 5.05 & 370.7 & "1,6" \\
\hline 5 & 40.6 & 10.50 & 10.89 & 2.29 & 2.81 & 3.38 & 4.14 & 9.94 & \\
\hline UGC 4368 & 56.7 & 13.57 & 10.21 & 21.52 & 17.81 & 3.11 & 4.84 & 369.8 & "1,6" \\
\hline 6 & 37.8 & 10.40 & 10.82 & 2.22 & 2.77 & 3.45 & 4.04 & 10.04 & \\
\hline UGC 4375 & 30.3 & 13.28 & 9.53 & 21.31 & 17.21 & 3.33 & 2.97 & 355.5 & "1,6" \\
\hline 5 & 50.1 & 10.04 & 10.55 & 2.31 & 2.93 & 3.62 & 2.49 & 9.26 & \\
\hline UGC 4422 & 63.2 & 12.85 & 8.83 & 22.04 & 18.38 & 3.75 & 9.51 & 606.5 & "1,6" \\
\hline 5 & 35.7 & 10.79 & 11.46 & 1.94 & 2.55 & 3.59 & 8.28 & 10.11 & \\
\hline UGC 5103 & 54.8 & 12.77 & 9.22 & 20.50 & 16.56 & 3.15 & 4.72 & 465.3 & "1,6" \\
\hline 3 & 54.0 & 10.76 & 11.19 & 2.58 & 3.15 & 3.50 & 3.85 & 9.98 & \\
\hline UGC 5303 & 20.9 & 12.24 & 8.67 & 21.32 & 17.89 & 3.14 & 3.73 & 330.0 & "1,6" \\
\hline 5 & 55.5 & 10.15 & 10.57 & 2.29 & 2.61 & 2.89 & 3.83 & 9.38 & \\
\hline UGC 5510 & 19.8 & 12.36 & 9.24 & 20.66 & 17.37 & 2.93 & 1.96 & 287.5 & "1,6" \\
\hline 5 & 36.8 & 9.94 & 10.28 & 2.56 & 2.96 & 3.05 & 1.77 & 9.42 & \\
\hline UGC 5554 & 18.7 & 12.88 & 9.10 & 20.98 & 16.94 & 3.47 & 1.73 & 355.3 & "1,6" \\
\hline 1 & 47.6 & 9.74 & 10.30 & 2.43 & 3.06 & 3.65 & 1.52 & 8.69 & \\
\hline UGC 5633 & 20.5 & 14.24 & 11.28 & 23.13 & 20.01 & 2.64 & 2.77 & 198.8 & "1,6" \\
\hline 8 & 52.4 & 9.27 & 9.50 & 1.43 & 1.80 & 2.99 & 2.93 & 9.19 & \\
\hline UGC 6028 & 16.9 & 13.14 & 10.19 & 20.49 & 17.27 & 2.60 & 1.25 & 233.6 & "1,6" \\
\hline 3 & 56.2 & 9.56 & 9.77 & 2.62 & 2.85 & 2.66 & 1.06 & 9.14 & \\
\hline UGC 6453 & 17.8 & 12.45 & 9.18 & 20.91 & 17.16 & 3.02 & 1.84 & 331.0 & "1,6" \\
\hline 4 & 42.5 & 9.84 & 10.22 & 2.46 & 3.01 & 3.44 & 1.41 & 9.47 & \\
\hline UGC 6460 & 17.7 & 11.87 & 8.62 & 20.66 & 17.15 & 3.03 & 2.37 & 273.9 & "1,6" \\
\hline 4 & 42.5 & 10.05 & 10.44 & 2.56 & 3.01 & 3.20 & 2.07 & 9.02 & \\
\hline UGC 6746 & 102.6 & 13.35 & 9.39 & 21.38 & 17.30 & 3.72 & 8.80 & 798.1 & "1,6" \\
\hline 0 & 41.6 & 11.01 & 11.67 & 2.21 & 2.93 & 3.89 & 7.96 & 9.94 & \\
\hline UGC 7169 & 32.4 & 12.62 & 9.63 & 20.11 & 16.55 & 2.76 & 2.08 & 347.3 & "1,6" \\
\hline 5 & 36.8 & 10.28 & 10.56 & 2.79 & 3.28 & 3.30 & 1.57 & 9.65 & \\
\hline UGC 7315 & 13.7 & 12.55 & 8.62 & 19.99 & 16.03 & 3.60 & 0.98 & 313.5 & $" 1,6 "$ \\
\hline 4 & 49.3 & 9.60 & 10.22 & 2.83 & 3.41 & 3.53 & 0.97 & 8.39 & \\
\hline UGC 7523 & 14.7 & 11.80 & 8.08 & 21.49 & 17.70 & 3.52 & 2.57 & 264.1 & "1,6" \\
\hline 3 & 36.8 & 9.91 & 10.49 & 2.25 & 2.83 & 3.52 & 2.32 & 8.55 & \\
\hline UGC 7901 & 13.0 & 11.46 & 7.90 & 20.07 & 16.06 & 3.16 & 1.58 & 477.9 & $" 1,6 "$ \\
\hline 5 & 49.3 & 10.03 & 10.46 & 2.79 & 3.40 & 3.59 & 1.24 & 9.39 & \\
\hline
\end{tabular}


Table 1. continued.

\begin{tabular}{|c|c|c|c|c|c|c|c|c|c|}
\hline $\begin{array}{l}\text { Name } \\
\mathrm{T} \\
\end{array}$ & $\begin{array}{l}\mathrm{D}^{\mathrm{a}} \\
i\left(^{o}\right) \\
\end{array}$ & $\begin{array}{c}m_{B}^{\mathrm{b}} \\
\log L_{B, c}^{\mathrm{g}}\end{array}$ & $\begin{array}{c}m_{K}^{\mathrm{b}} \\
\operatorname{Lg} L_{K, c}^{\mathrm{g}}\end{array}$ & $\begin{array}{c}\mu_{0, B}^{\mathrm{c}} \\
\operatorname{Lg} \Sigma_{0, B c}^{\mathrm{h}} \\
\end{array}$ & $\begin{array}{c}\mu_{0, K}^{\mathrm{c}} \\
\operatorname{Lg} \Sigma_{0, K c}^{\mathrm{h}} \\
\end{array}$ & $\begin{array}{l}(B-K)^{\mathrm{d}} \\
(B-K)_{0}^{\mathrm{i}}\end{array}$ & $\begin{array}{l}h_{B}^{\mathrm{e}} \\
h_{K}^{\mathrm{e}} \\
\end{array}$ & $\begin{array}{c}\mathrm{W}_{20, c}^{\mathrm{f}} \\
\mathrm{LgM}_{\mathrm{HI}}^{\mathrm{j}} \\
\end{array}$ & Ref. $^{\mathrm{k}}$ \\
\hline UGC 8279 & 39.7 & 13.07 & 9.74 & 20.52 & 16.90 & 3.16 & 2.62 & 369.8 & $" 1,6 "$ \\
\hline 4 & 40.6 & 10.25 & 10.69 & 2.58 & 3.12 & 3.41 & 2.21 & 9.60 & \\
\hline UGC 8865 & 36.9 & 12.62 & 8.98 & 21.89 & 18.27 & 3.41 & 5.73 & 422.4 & "1,6" \\
\hline 2 & 41.6 & 10.39 & 10.93 & 1.96 & 2.56 & 3.59 & 5.24 & 9.62 & \\
\hline UGC 9481 & 56.7 & 13.31 & 10.00 & 21.22 & 18.03 & 3.14 & 4.84 & 322.2 & "1,6" \\
\hline 4 & 43.4 & 10.47 & 10.90 & 2.29 & 2.64 & 2.95 & 4.81 & 10.08 & \\
\hline UGC 9926 & 30.3 & 12.17 & 8.45 & 20.13 & 16.45 & 3.28 & 2.63 & 461.4 & "1,6" \\
\hline 5 & 46.0 & 10.50 & 10.98 & 2.81 & 3.27 & 3.22 & 2.44 & 9.46 & \\
\hline UGC 9943 & 30.1 & 12.30 & 8.67 & 20.40 & 16.60 & 3.19 & 2.91 & 401.9 & "1,6" \\
\hline 5 & 51.7 & 10.44 & 10.89 & 2.67 & 3.16 & 3.31 & 2.56 & 9.60 & \\
\hline UGC 10083 & 28.7 & 12.37 & 9.19 & 21.51 & 17.60 & 2.98 & 3.24 & 270.5 & "1,6" \\
\hline 2 & 35.7 & 10.26 & 10.63 & 2.24 & 2.87 & 3.64 & 2.63 & 9.65 & \\
\hline UGC 10445 & 16.8 & 13.10 & 10.81 & 21.76 & 19.00 & 2.07 & 1.65 & 216.2 & "1,6" \\
\hline 6 & 40.6 & 9.51 & 9.51 & 2.05 & 2.29 & 2.66 & 1.59 & 9.25 & \\
\hline UGC 11628 & 61.6 & 12.82 & 8.54 & 22.27 & 17.19 & 3.63 & 11.60 & 659.9 & "1,6" \\
\hline 2 & 46.8 & 10.95 & 11.58 & 2.01 & 2.96 & 4.46 & 6.55 & 10.23 & \\
\hline UGC 11872 & 18.1 & 12.03 & 8.11 & 20.46 & 15.71 & 3.44 & 1.61 & 420.4 & "1,6" \\
\hline 3 & 43.4 & 10.12 & 10.67 & 2.72 & 3.59 & 4.23 & 1.11 & 9.31 & \\
\hline UGC 12343 & 35.2 & 11.87 & 7.81 & 21.95 & 17.67 & 3.45 & 8.65 & 544.2 & "1,6" \\
\hline 4 & 40.6 & 10.82 & 11.37 & 2.18 & 2.82 & 3.67 & 6.77 & 10.04 & \\
\hline UGC 12511 & 52.0 & 13.81 & 10.58 & 22.46 & 18.35 & 2.98 & 6.15 & 434.6 & "1,6" \\
\hline 6 & 35.7 & 10.23 & 10.59 & 1.78 & 2.56 & 4.02 & 3.33 & 10.32 & \\
\hline UGC 12614 & 51.8 & 12.64 & 9.12 & 20.94 & 17.24 & 3.15 & 5.43 & 420.1 & "1,6" \\
\hline 5 & 44.2 & 10.75 & 11.18 & 2.46 & 2.956 & 3.32 & 5.08 & 9.92 & \\
\hline UGC 12638 & 82.0 & 13.95 & 9.99 & 22.17 & 18.27 & 3.60 & 8.51 & 469.8 & "1,6" \\
\hline 5 & 36.8 & 10.62 & 11.23 & 1.92 & 2.59 & 3.74 & 7.88 & 10.03 & \\
\hline UGC 12654 & 59.5 & 13.65 & 10.28 & 21.76 & 17.98 & 3.08 & 5.65 & 354.2 & $" 1,6 "$ \\
\hline 4 & 35.7 & 10.43 & 10.83 & 2.16 & 2.71 & 3.45 & 4.38 & 9.85 & \\
\hline UGC 6399 & 14.6 & 14.33 & 11.09 & 21.83 & 18.72 & 2.79 & 1.69 & 172.0 & "2,6" \\
\hline 9 & 75.0 & 9.00 & 9.29 & 1.56 & 1.96 & 3.07 & 1.86 & 9.03 & \\
\hline UGC 6446 & 12.5 & 13.52 & 11.50 & 22.61 & 19.31 & 1.86 & 2.28 & 174.0 & "2,6" \\
\hline 7 & 51.0 & 9.08 & 8.99 & 1.59 & 2.08 & 3.30 & 1.49 & 9.19 & \\
\hline NGC3726 & 15.2 & 11.00 & 7.96 & 21.07 & 17.19 & 2.74 & 4.30 & 331.0 & "2,6" \\
\hline 5 & 53.0 & 10.32 & 10.60 & 2.37 & 2.93 & 3.48 & 2.79 & 9.82 & \\
\hline NGC3769 & 13.5 & 12.80 & 9.10 & 19.93 & 16.34 & 3.13 & 1.26 & 256.0 & "2,6" \\
\hline 3 & 70.0 & 9.64 & 10.06 & 2.76 & 2.98 & 2.62 & 1.30 & 9.37 & \\
\hline NGC3877 & 15.9 & 11.91 & 7.75 & 19.72 & 15.50 & 3.20 & 2.60 & 335.0 & "2,6" \\
\hline 5 & 76.0 & 10.31 & 10.76 & 2.81 & 3.16 & 2.93 & 2.41 & 9.21 & \\
\hline NGC3893 & 17.0 & 11.20 & 7.84 & 19.88 & 16.71 & 3.07 & 2.13 & 382.0 & "2,6" \\
\hline 5 & 49.0 & 10.34 & 10.74 & 2.86 & 3.16 & 2.82 & 2.23 & 9.75 & \\
\hline NGC3917 & 17.1 & 12.66 & 9.08 & 20.59 & 17.12 & 2.80 & 2.69 & 276.0 & "2,6" \\
\hline 6 & 79.0 & 9.98 & 10.28 & 1.99 & 2.55 & 3.46 & 2.84 & 9.26 & \\
\hline NGC3949 & 14.5 & 11.55 & 8.43 & 19.54 & 16.55 & 2.85 & 1.31 & 321.0 & $" 2,6 "$ \\
\hline 4 & 55.0 & 10.06 & 10.38 & 2.99 & 3.20 & 2.60 & 1.35 & 9.31 & \\
\hline NGC3953 & 18.4 & 11.03 & 7.03 & 20.43 & 16.47 & 3.47 & 3.85 & 446.0 & "2,6" \\
\hline 4 & 62.0 & 10.59 & 11.16 & 2.62 & 3.14 & 3.37 & 3.79 & 9.41 & \\
\hline NGC3972 & 15.5 & 13.09 & 9.39 & 20.23 & 16.50 & 3.10 & 1.71 & 264.0 & "2,6" \\
\hline 4 & 77.0 & 9.66 & 10.07 & 2.62 & 2.87 & 2.71 & 1.62 & 8.95 & \\
\hline UGC 6917 & 16.2 & 13.15 & 10.30 & 22.27 & 19.17 & 2.49 & 2.93 & 224.0 & "2,6" \\
\hline 9 & 56.0 & 9.526 & 9.69 & 1.68 & 2.06 & 3.02 & 2.55 & 9.26 & \\
\hline UGC 6923 & 18.6 & 13.91 & 11.04 & 21.42 & 18.34 & 2.54 & 1.62 & 160.0 & "2,6" \\
\hline 10 & 65.0 & 9.33 & 9.51 & 1.91 & 2.28 & 3.01 & 1.57 & 8.92 & \\
\hline NGC3992 & 18.3 & 10.86 & 7.23 & 20.29 & 16.82 & 3.14 & 4.05 & 547.0 & "2,6" \\
\hline 4 & 56.0 & 10.65 & 11.07 & 2.69 & 3.05 & 2.97 & 4.11 & 9.76 & \\
\hline UGC 6983 & 18.8 & 13.10 & 10.52 & 22.59 & 19.41 & 2.36 & 3.60 & 221.0 & "2,6" \\
\hline 6 & 49.0 & 9.63 & 9.74 & 1.64 & 2.07 & 3.15 & 2.67 & 9.51 & \\
\hline
\end{tabular}


Table 1. Continued.

\begin{tabular}{|c|c|c|c|c|c|c|c|c|c|}
\hline $\begin{array}{l}\text { Name } \\
\mathrm{T}\end{array}$ & $\begin{array}{l}\mathrm{D}^{\mathrm{a}} \\
i\left(^{o}\right)\end{array}$ & $\begin{array}{c}m_{B}^{\mathrm{b}} \\
\log L_{B, c}^{\mathrm{g}}\end{array}$ & $\begin{array}{c}m_{K}^{\mathrm{b}} \\
\operatorname{Lg} L_{K, c}^{\mathrm{g}}\end{array}$ & $\begin{array}{c}\mu_{0, B}^{\mathrm{c}} \\
\operatorname{Lg} \sum_{0, B c}^{\mathrm{h}}\end{array}$ & $\begin{array}{c}\mu_{0, K}^{\mathrm{c}} \\
\operatorname{Lg} \Sigma_{0, K c}^{\mathrm{h}}\end{array}$ & $\begin{array}{l}(B-K)^{\mathrm{d}} \\
(B-K)_{0}^{\mathrm{i}}\end{array}$ & $\begin{array}{l}h_{B}^{\mathrm{e}} \\
h_{K}^{\mathrm{e}} \\
\end{array}$ & $\begin{array}{c}\mathrm{W}_{20, c}^{\mathrm{f}} \\
\mathrm{LgM}_{\mathrm{HI}}^{\mathrm{j}}\end{array}$ & Ref. $^{k}$ \\
\hline NGC4051 & 13.1 & 10.98 & 7.86 & 20.78 & 16.90 & 2.93 & 2.94 & 308.0 & “"2,6” \\
\hline 4 & 49.0 & 10.17 & 10.51 & 2.48 & 3.09 & 3.58 & 2.10 & 9.26 & \\
\hline NGC4088 & 14.1 & 11.23 & 7.46 & 19.77 & 16.19 & 3.11 & 2.33 & 362.0 & $" 2,6 "$ \\
\hline 5 & 69.0 & 10.33 & 10.75 & 2.84 & 3.11 & 2.74 & 2.54 & 9.72 & \\
\hline NGC4100 & 18.6 & 11.91 & 8.02 & 19.82 & 15.77 & 3.07 & 2.81 & 386.0 & $" 2,6 "$ \\
\hline 4 & 73.0 & 10.39 & 10.79 & 2.80 & 3.18 & 3.03 & 2.54 & 9.63 & \\
\hline NGC4102 & 15.4 & 12.04 & 7.86 & 19.28 & 15.82 & 3.80 & 1.30 & 393.0 & $" 2,6 "$ \\
\hline 3 & 56.0 & 9.97 & 10.66 & 3.08 & 3.45 & 3.00 & 1.34 & 8.75 & \\
\hline NGC3718 & 17.5 & 11.28 & 7.47 & 21.88 & 17.52 & 3.17 & 6.83 & 476.0 & “2,6" \\
\hline 1 & 69.0 & 10.50 & 10.94 & 2.00 & 2.63 & 3.66 & 4.79 & 9.86 & \\
\hline NGC3729 & 18.0 & 12.31 & 8.60 & 20.22 & 16.44 & 3.53 & 1.67 & 296.0 & "2,6" \\
\hline 1 & 49.0 & 9.89 & 10.48 & 2.70 & 3.27 & 3.50 & 1.62 & 9.14 & \\
\hline UGC 6773 & 16.4 & 14.42 & 11.23 & 21.67 & 18.79 & 3.06 & 1.19 & 112.0 & $" 2,6 "$ \\
\hline 10 & 58.0 & 8.92 & 9.32 & 1.90 & 2.21 & 2.84 & 1.33 & 8.55 & \\
\hline UGC 6818 & 14.6 & 14.43 & 11.70 & 21.62 & 18.68 & 2.33 & 1.49 & 151.0 & "2,6" \\
\hline 7 & 75.0 & 8.94 & 9.05 & 1.65 & 1.97 & 2.88 & 1.41 & 8.90 & \\
\hline NGC3985 & 16.7 & 13.25 & 10.19 & 20.04 & 17.06 & 2.82 & 0.87 & 180.0 & "2,6" \\
\hline 9 & 51.0 & 9.46 & 9.75 & 2.79 & 2.97 & 2.52 & 1.02 & 8.84 & \\
\hline UGC 7089 & 14.0 & 13.73 & 11.11 & 21.51 & 18.73 & 2.21 & 2.24 & 138.0 & "2,6" \\
\hline 8 & 80.0 & 9.19 & 9.24 & 1.51 & 1.78 & 2.74 & 2.32 & 9.23 & \\
\hline UGC 7094 & 14.1 & 14.74 & 11.58 & 21.99 & 18.56 & 3.12 & 1.31 & 76.0 & $" 2,6 "$ \\
\hline 8 & 70.0 & 8.62 & 9.04 & 1.70 & 2.24 & 3.40 & 1.10 & 8.75 & \\
\hline NGC4117 & 16.3 & 14.05 & 9.98 & 21.16 & 17.80 & 3.60 & 1.04 & 285.0 & $" 2,6 "$ \\
\hline-2 & 68.0 & 9.22 & 9.83 & 2.01 & 2.52 & 3.33 & 1.18 & 8.97 & \\
\hline NGC4138 & 15.9 & 12.27 & 8.19 & 20.05 & 15.98 & 3.75 & 1.25 & 374.0 & $" 2,6 "$ \\
\hline-1 & 53.0 & 9.85 & 10.52 & 2.77 & 3.40 & 3.65 & 1.20 & 8.71 & \\
\hline NGC4218 & 13.5 & 13.69 & 10.83 & 20.35 & 16.74 & 2.69 & 0.63 & 150.0 & “2,6" \\
\hline 1 & 53.0 & 9.07 & 9.31 & 2.65 & 3.08 & 3.14 & 0.51 & 8.49 & \\
\hline NGC4220 & 16.4 & 12.34 & 8.36 & 20.08 & 15.27 & 3.18 & 1.96 & 399.0 & $" 2,6 "$ \\
\hline 0 & 78.0 & 10.09 & 10.54 & 2.69 & 3.40 & 3.84 & 1.38 & 8.99 & \\
\hline UGC 128 & 67.8 & 15.16 & 12.10 & 23.55 & 20.30 & 2.75 & 7.98 & 336.6 & $" 3,4 "$ \\
\hline 8 & 36.0 & 9.94 & 10.22 & 1.37 & 1.78 & 3.09 & 6.90 & 9.99 & \\
\hline F563-V2 & 69.6 & 16.25 & 13.80 & 22.16 & 19.29 & 2.37 & 2.63 & 152.5 & $" 3,4 "$ \\
\hline 10 & 45.1 & 9.43 & 9.55 & 1.79 & 2.12 & 2.90 & 2.16 & 9.46 & \\
\hline F568-3 & 93.5 & 16.12 & 13.11 & 22.33 & 19.16 & 2.90 & 4.03 & 241.1 & "3,4" \\
\hline 7 & 39.7 & 9.76 & 10.09 & 1.75 & 2.20 & 3.21 & 3.62 & 9.60 & \\
\hline F574-1 & 106.0 & 16.67 & 13.80 & 23.00 & 19.66 & 2.55 & 5.91 & 196.5 & “3,4" \\
\hline 7 & 67.9 & 9.74 & 9.94 & 1.20 & 1.73 & 3.39 & 4.26 & 9.61 & \\
\hline F583-1 & 38.3 & 16.40 & 13.50 & 23.05 & 19.80 & 2.50 & 2.30 & 173.3 & "3,4" \\
\hline 9 & 65.7 & 8.99 & 9.16 & 1.31 & 1.73 & 3.12 & 1.49 & 9.40 & \\
\hline MilkyWay & - & - & - & - & - & 3.45 & 5.00 & 440.0 & "9,10" \\
\hline 4 & - & 10.28 & 10.83 & 1.98 & 3.00 & 4.62 & 3.00 & 9.60 & \\
\hline Andromeda & 0.7 & 4.36 & - & - & 15.67 & 3.49 & 6.40 & 525.4 & "7,8,9" \\
\hline 3 & 75.2 & 10.70 & 11.27 & 2.29 & 3.24 & 4.46 & 4.10 & 2.72 & \\
\hline 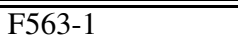 & 499.0 & 15.60 & - & 23.53 & - & - & 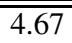 & $\bar{~} 118.6$ & "4" \\
\hline 9 & 52.4 & 9.38 & - & 1.20 & - & - & - & - & \\
\hline F563-V1 & 54.8 & 16.93 & - & 23.54 & - & - & 2.58 & 77.0 & "4" \\
\hline 10 & 42.5 & 8.91 & - & 1.26 & - & - & - & - & \\
\hline F565-V2 & 51.9 & 17.58 & - & 23.98 & - & - & 2.90 & 97.1 & "4" \\
\hline 10 & 65.0 & 8.62 & - & 0.87 & - & - & - & - & \\
\hline F568-V1 & 87.0 & 16.67 & - & 23.01 & - & - & 3.46 & 273.9 & "4" \\
\hline 8 & 35.7 & 9.43 & - & 1.47 & - & - & - & - & \\
\hline F571-V1 & 85.6 & 17.40 & - & 23.78 & - & - & 3.46 & 143.8 & "4" \\
\hline 8 & 35.7 & 9.10 & - & 1.16 & - & - & - & - & \\
\hline ESO-LV1150280 & 91.3 & 14.32 & - & 21.19 & - & - & 4.86 & 212.0 & “5,6,11" \\
\hline 4 & 53.2 & 10.52 & - & 2.14 & - & - & - & - & \\
\hline
\end{tabular}


Table 1. continued.

\begin{tabular}{lccccccccc}
\hline \hline Name & $\mathrm{D}^{\mathrm{a}}$ & $m_{B}^{\mathrm{b}}$ & $m_{K}^{\mathrm{b}}$ & $\mu_{0, B}^{\mathrm{c}}$ & $\mu_{0, K}^{\mathrm{c}}$ & $(B-K)^{\mathrm{d}}$ & $h_{B}^{\mathrm{e}}$ & $\mathrm{W}_{20, c}^{\mathrm{f}}$ & Ref." \\
$\mathrm{T}$ & $i\left(^{(}\right)$ & $\log L_{B, c}^{\mathrm{g}}$ & $\operatorname{Lg} L_{K, c}^{\mathrm{g}}$ & $\operatorname{Lg} \sum_{0, B c}^{\mathrm{h}}$ & $\operatorname{Lg} \sum_{0, K c}^{\mathrm{h}}$ & $(B-K)_{0}^{\mathrm{i}}$ & $h_{K}^{\mathrm{e}}$ & $\mathrm{LgM}_{\mathrm{HI}}^{\mathrm{j}}$ & \\
\hline ESO-LV1530170 & 90.9 & 13.99 & - & 23.07 & - & - & 12.92 & 246.9 & “5,6,11" \\
5 & 45.9 & 10.62 & - & 1.43 & - & - & - & - & \\
\hline ESO-LV2520100 & 140.0 & 14.20 & - & 22.69 & - & - & 13.09 & 621.1 & “5,6,11" \\
2 & 41.6 & 10.89 & - & 1.55 & - & - & - & - & \\
\hline ESO-LV3500110 & 221.1 & 14.49 & - & 22.72 & - & - & 15.73 & 404.0 & “5,6,11" \\
1 & 40.6 & 11.11 & - & 1.48 & - & - & - & - & \\
\hline ESO-LV5520190 & 173.6 & 15.07 & - & 22.75 & - & - & 17.24 & 545.7 & “5,6,11" \\
2 & 53.2 & 10.84 & - & 1.46 & - & - & - & - & \\
\hline ESO-LV1220040 & 139.4 & 15.47 & - & 23.96 & - & - & 26.91 & 112.5 & “5,6,11" \\
3 & 52.4 & 10.45 & - & 1.11 & - & - & - & - & \\
\hline ESO-LV3740090 & 39.9 & 15.45 & - & 23.40 & - & - & 3.23 & 112.2 & “5,6,11" \\
1 & 36.8 & 9.38 & - & 1.51 & - & - & - & - & \\
\hline
\end{tabular}

a Luminous distance in Mpc.

b Raw uncorrected apparent magnitude.

c Raw uncorrected disk central SB (magarcsec $\left.{ }^{-2}\right)$.

d Corrected integral $(B-K)$ color.

e Disk scale radius in kpc.

f Corrected HI equivalent line-width at $20 \%$ level of intensity $(\mathrm{km} / \mathrm{s})$.

g Corrected total luminosity (in $L_{B \odot}$ and $L_{K \odot}$, respectively).

h Corrected disk central SB (in $L_{B \odot} \mathrm{pc}^{-2}$ and $L_{K \odot} \mathrm{pc}^{-2}$, respectively).

i Corrected disk central $(B-K)$ color.

j $\mathrm{HI}$ mass in $M_{\odot}$.

k References: 1.- de Jong (1996a, 1996b). 2.- Verheijen (1997); Verheijen \& Sancisi (2001). 3.- Bell et al. (2000). 4.- de Blok et al.(1995,1996,2001). 5.- Beijersbergen et al. (1999). 6.- LEDA. 7.- Cox 2000. 8.- Hiromoto et al. (1983). 9.- Gilmore et al. (1990). 10.- Kent et al. (1991). 11.- Graham (2002). 